\title{
The hydrodynamics of confined dispersions
}

\author{
James W. Swan $\dagger+$ and John F. Brady \\ Division of Chemistry and Chemical Engineering, California Institute of Technology, Pasadena, \\ CA 91125, USA
}

(Received 14 September 2010; revised 10 June 2011; accepted 17 August 2011;

first published online 17 October 2011)

A method is proposed for computing the low-Reynolds-number hydrodynamic forces on particles comprising a suspension confined by two parallel, no-slip walls. This is constructed via the two-dimensional analogue of Hasimoto's solution (J. Fluid Mech., vol. 5, 1959, pp. 317-328) for a periodic array of point forces in a viscous, incompressible fluid, and, like Hasimoto, the summation of interactions is accelerated by substitution and superposition of 'Ewald-like' forcing. This method is akin to the accelerated Stokesian dynamics technique (J. Fluid Mech., vol. 448, 2001, pp. 115-146) and models the suspension dynamics with log-linear computational scaling. The effectiveness of this approach is demonstrated with a calculation of the high-frequency dynamic viscosity of a colloidal dispersion as function of volume fraction and channel width. Similarly, the short-time self-diffusivity for and the sedimentation rate of spherical particles in a confined suspension are determined. The results demonstrate the influence of confining geometry on the transport of small particles, which is becoming increasingly important for micro- and biofluidics.

Key words: colloids, computational methods, Stokesian dynamics

\section{Introduction}

The dynamics of micrometre and sub-micrometre sized particles suspended in a viscous fluid hold many practical consequences for quotidian living and scientific study. While the honey poured into a cup of tea is Newtonian, the butter lathered on a scone certainly is not. Many paints are dispersions of nanoparticles in a suspending medium such as water or latex, while the interiors of cells are suspensions of proteins and other macromolecules, which govern the fate of all living organisms. Each and every interaction, no matter the origin, is mediated by the fluid surrounding the suspended particles, and in all these cases - butter spreading, paint running and proteins folding - the dynamics are mediated not just by the interaction of the suspended colloids with each other but also by the interactions of the colloids with their boundaries. In fact, as dissipative systems of ever smaller dimension - e.g. the interior of cells (Daniels, Masi \& Wirtz 2006), microfluidic separators (Ashton, Padala \& Kane 2003) and viral packaging (Ali, Marenduzzo \& Yeomans 2004) - are investigated, the importance of the boundary-mediated interactions may become

\section{$\dagger$ Email address for correspondence: jswan@ @altech.edu}

$\$$ Present address: Department of Chemical Engineering and Center for Molecular and Engineering Thermodynamics, University of Delaware, 150 Academy St., Newark, DE 19716, USA. 
orders of magnitude more important. One role that computation can play in these investigations is in determining the degree of influence that the bounding geometry has on the dynamics of particles in suspension. As a model system, consider a suspension of infinite extent bound in one dimension between the parallel, no-slip walls of a channel. The suspension comprises rigid, no-slip, spherical particles of radii $a$ and an embedding fluid with Newtonian shear viscosity $\eta$. While the present methodology can be generalized to any geometry, the parallel channel and spherical particle form a relatively simple model that is scientifically relevant and a general representation of bounded complex fluids.

The study of colloidal particles in a bounded geometry, in particular the channel geometry, has a long history. A brief survey of prior approaches and the current state of the art analytically, computationally and experimentally are offered with the intention of providing some scope to the approach advanced in this paper.

The calculation of the hydrodynamic resistance to the motion of a single spherical particle between parallel walls by Faxén (1921) used a 'method of reflections' approach to generate a series solution in inverse powers of the channel width for particles located one-half and one-quarter of the way across the channel. His approach can certainly be applied to particles located elsewhere in the channel, but the fortuitous symmetry that Faxén used breaks down and makes the calculation much more difficult (see Swan \& Brady 2010). In search of a more general expression, Oseen (1928) proposed considering the channel walls independently and superimposing the resistance due to each. While not correct, this can often be a suitable approximation. For instance, Guth \& Simha (1936) repeated Einstein's calculation of the viscosity of a dilute suspension while bounding the suspension by two superimposable no-slip walls. They recover the correct scaling for the viscosity increment with respect to the channel width. Blake (1971) brought an analogous electrostatic approach to the problem by determining the Green's function for Stokes flow above a single no-slip wall. This same technique was employed by Liron \& Mochon (1976) for computing the Green's function for Stokes flow in the parallel-wall geometry. Key to this work was the conclusion that, while the Green's function with a single wall requires a finite number of 'reflections', the parallel-wall Green's function must be represented as an infinitude of reflections. This of course poses serious challenges to modelling suspensions in channels accurately. Since Stokes flows are unique, any solution to this problem will in some sense require the computation of this infinite sum.

It appears that this difficulty can only be overcome with some form of computational approach. After all, for a single particle in even the simplest channel geometry, the hydrodynamic interactions are among three bodies - never an easy computation. A number of different models for the dynamics of a dispersion in a channel have arisen. Durlofsky \& Brady (1989) combined their Stokesian dynamics algorithm with a discretized model of the channel walls that accounts for the additional energy dissipation due to the no-slip condition on the walls. Similarly, Nott \& Brady (1994) modelled the walls as an array of particles fixed in space. While qualitatively correct, this approach presents a number of physical and computational challenges, such as the 'leakiness' of the walls and possible errors arising from the pairwise superposition of lubrication interactions with the wall particles. An approach using Stokes flow eigenfunctions was introduced by Bhattacharya, Blawzdziewicz \& Wajnryb (2005) and has had success in modelling suspensions in channels, and Jones (2004) used this technique to model the motion of a single particle due to a Poiseuille flow. Similarly, Zurita-Gotor, Blawzdziewicz \& Wajnryb (2007) extended this model to study the rheology of rod-like particles in suspension. One other approach originated 
by Staben, Zinchenko \& Davis (2003) relies on the boundary-integral formulation for Stokes flow and employs the Green's function for channel flow (Liron \& Mochon 1976) in the computation of the hydrodynamic resistance to the motion of a particle in a channel. These studies represent three distinct classes of technique: wall discretization, eigenfunction expansion, and boundary-integral solution. A survey of the literature will show that virtually all computational studies of dispersions between walls are rooted in one of these classes. The approach here returns to the electrostatic analogy and utilizes the Fourier transformation of the Stokes flow solution and an Ewald summation to generate a $\log$-linear algorithm for the dynamics of particles in a channel. Another study worth mentioning is that of Hernández-Ortiz, de Pablo \& Graham (2007), which proposes a novel method for dividing the force density in the fluid into so-called 'local' and 'global' contributions. In the development of the present model, a similar splitting arises and is justified analytically as a direct corollary to the Ewald summation technique (see e.g. Beenakker 1986).

There have been many experimental investigations of the macroscopic properties of channel-confined suspensions (Goldsmith \& Mason 1962; Karnis, Goldsmith \& Mason 1966; Seshadri \& Sutera 1970; Cox \& Mason 1971; Cox \& Hsu 1977; de Gennes 1979; Gregory 1981), perhaps culminating in Leighton \& Acrivos (1987), where a direct connection to the particle micromechanics explained the observation of a time-dependent viscosity in a Couette flow device. This showed that detailed hydrodynamic and particle-particle interactions cannot be marginalized in models of confined viscoelastic materials. The experiments of Koh, Hookham \& Leal (1994) for suspensions in rectangular channels were the first to measure detailed distributions of the spatial variation in particle concentration and velocity for pressure-driven flows, while Lyon \& Leal (1998) tested the diffusive flux (Leighton \& Acrivos 1987) and suspension balance (Nott \& Brady 1994) continuum models that incorporate this principle. Still, such models require knowledge of physical parameters based on the local suspension dynamics and rheology, which have been pursued extensively (see e.g. Butler \& Bonnecaze 1999; Dufresne, Altman \& Grier 2001; Zarraga \& Leighton 2002; Frank et al. 2003; Norman, Nayak \& Bonnecaze 2005; Ramachandran \& Leighton 2007). While parameters for continuum models have been refined, these experiments largely represent measurements of long-time suspension properties.

In the following sections, a method is developed for computing the hydrodynamic forces on the particles comprising a suspension bound in one dimension between parallel channel walls. Principally, we compute the Green's functions for the Stokes equations,

$$
\eta \nabla^{2} \boldsymbol{u}=\nabla p-\boldsymbol{f}(\boldsymbol{x}), \quad \boldsymbol{\nabla} \cdot \boldsymbol{u}=0,
$$

governing the hydrodynamics of particles in fluids characterized by zero Reynolds number $(\rho U L / \eta)$ and subject to a no-slip condition on the channel walls and periodic boundary conditions transverse to the walls. Here, $\boldsymbol{u}$ and $p$ are the fluid velocity and pressure, respectively, and $\boldsymbol{f}(\boldsymbol{x})$ is a body force acting on the fluid; while $\rho$ and $\eta$ are the fluid density and viscosity, and $U$ and $L$ are velocity and length scales characteristic of the physical situation being modelled. The Green's function is determined via Fourier transformation and is therefore a sum over all the Fourier modes. The coefficients corresponding to these modes have a simple closed form, though the convergence of the series is known to require many terms, as the velocity field due to a point force in a channel decays like $r^{-2}$, where $r$ is the distance from the force itself. As such, the Fourier series is unsuitable for rapid numerical simulations. 
Instead, we introduce a frequency filter of the Ewald summation form, i.e.

$$
\left(1+\pi \alpha \xi^{2}\right) \mathrm{e}^{-\pi \alpha \xi^{2}},
$$

where $\xi$ is the three-dimensional Fourier space variable and $\alpha$ is the so-called splitting parameter. Tuning the value of $\alpha$ establishes the maximal frequency included in the Fourier series summation. As merely filtering the high-frequency components alone may not be sufficiently accurate, we find the solution to the Stokes equations for the removed components in real space to restore fidelity to the computation. The real-space solution is computed in closed form by applying the superposition approximation to the channel walls. However, the high-frequency components of the flow decay, without exception, in a Gaussian fashion. As such, the superposition approximation introduces at most an exponentially small error. In this way, the Green's function for Stokes flow in a parallel-wall channel may be computed rapidly. Application of multipole expansions and Faxén formulae allows for the determination of the particle dynamics. The accelerated Stokesian dynamics algorithm is implemented and enables the calculation of the hydrodynamic forces on $N$ particles in $O(N \log N)$ computations.

This method is applied to the determination of the high-frequency dynamic viscosity of, the short-time self-diffusivity in, and the sedimentation rate of suspensions confined by parallel channel walls. We find that the high-frequency dynamic viscosity is insensitive to the effects of confinement. In particular, over the range of volume fractions investigated $(0.1<\phi<0.4)$, suspensions in channels as small as six particle radii across exhibit no more than a $15 \%$ deviation from the value of the highfrequency dynamic viscosity in the bulk. The viscosity is dominated by the solvent viscosity in the dilute regime, so that the effects of the particles alone (i.e. the Einstein viscosity increment) and the walls are masked. In the concentrated regime, the energy dissipated by the channel walls is not much more than that dissipated by the concentrated suspension surrounding an equivalent volume in an unbounded suspension. That is, to a concentrated suspension, the channel walls might as well be other densely packed particles. Therefore, the viscosity in confinement deviates little from the expected value for an unbounded suspension. In very dense suspensions, other physical processes (e.g. ordering) may alter these conclusions. The methodology proposed is still appropriate for these dense suspensions, however.

For the short-time self-diffusivity, we observe an array of behaviours relative to that of an unbounded suspension that depend in detail on the particle volume fraction and the channel width. In cases where the channel is wide $(12$ or more particle radii) and the suspension dilute, the diffusivity predicted via our method is near that expected for a single particle in a Newtonian fluid with a viscosity comparable to that of the suspension itself. This is a mean-field-type approximation. However, the computed diffusivity is always larger than the mean-field approximation because such an approximation does not account for the screening of hydrodynamic interactions among the particles. As such, narrowing the channel while maintaining a constant suspension volume fraction results in further discrepancy between the simulation's prediction and the mean-field approximation. The mean-field approximation presented is empirical and should not be confused with the results of a detailed physical analysis. Contrarily, particles in concentrated suspensions and wide channels have short-time self-diffusivities nearly equal to that of a particle in a bulk suspension of identical volume fraction. The diffusivity of the confined particle is always smaller than an unconfined one under these condition though. This additional hindrance can 
be understood as the effect of the additional energy dissipation due to the no-slip condition at the channel walls. Naturally, as the channel narrows, the difference grows.

The short-time sedimentation rate of suspensions confined between parallel walls (that due to an equilibrium hard-sphere distribution of particles) is dependent on the channel width and particle volume fraction as well as the end conditions on the channel. That is, is the channel left open such that fluid may enter and pour freely from it? This condition implies external control of the pressure at the ends of the channel. Or, is the channel sealed such that the mass in the channel is conserved? This condition implies that there is no mean volume flux through any plane normal to the channel walls. In the either case, we find that the suspension falls with roughly a parabolic velocity profile when dilute (10\% and $20 \%$ particle fraction by volume) and a plug flow profile when concentrated (30\% and $40 \%$ particle fraction by volume). The change in the total particle flux differs with change in volume fraction should the channel be left open or sealed. In the former case, 'heavier' suspensions (i.e. those more laden with particles) fall faster, while the opposite is true when the channel is sealed. This is easily understood, because, when the channel is left open, fluid is free to flow with the particles. Since the net force on the system (fluid and particles) is larger in more concentrated suspensions, the particles fall more quickly. When the channel is sealed, the fluid must move against the particles to yield a zero volume flux. This is termed 'back-flow', and, as is typical of unbounded suspensions, the back-flow is more effective at hindering concentrated suspensions.

This paper is structured as follows. In $\S 2$ a method is developed for rapidly computing the far-field hydrodynamic interactions among the particles comprising a periodic suspension bound by two parallel channel walls. The details of the calculations are confined to $\S 2.1-2.5$. In particular, $\$ 2.6$ describes the Stokesian dynamics method (Brady et al. 1988) for computing both the far-field and the near-field hydrodynamic forces and the velocities of particles in a suspension. Also discussed is the implementation of an accelerated Stokesian dynamics simulation (Sierou \& Brady 2001) based on the far-field interactions among particles in a channel. A detailed study of several canonical rheological measurements as a function of channel width and suspension concentration is made in $\S 3$. The less engaged reader may proceed directly to $\S 3$ for a detailed explanation of many applications of the developed method to the dynamics and rheology of confined dispersions.

\section{The hydrodynamics of particles in a channel}

This section details the solution of the Stokes equations subject to an arbitrary, periodic body force in a no-slip channel. Drawing from the Ewald summation technique yields a means to ensure rapid summation of this solution. As part of this rapid summation, a 'local' velocity field is resolved separately by considering the two walls as independent and assessing the decay rate of the resulting reflection flow. The body force is redefined as that due to a set of particles in the fluid, and it is shown how one may use these velocity fields to construct the grand mobility tensor, which is the essential linear coupling between velocity and force.

\subsection{The grand mobility tensor}

Because the Stokes equations are linear, the resulting velocity fields and tractions are linear functions of the boundary data. This has important consequences for the dynamics of small particles in a viscous fluid. Perhaps the most useful result of this simple fact is the existence of a grand mobility tensor that couples linearly all the 
moments of the hydrodynamic force density (force, $\boldsymbol{F}^{H}$; torque, $\boldsymbol{L}^{H}$; stresslet, $\boldsymbol{S}^{H}$; etc.) on the surface of particles suspended in the fluid to the moments of the particle velocities (translational, $\boldsymbol{U}$; rotational, $\boldsymbol{\Omega}$; rate of deformation, $\boldsymbol{E}$; etc.) such that

$$
\left(\begin{array}{c}
\boldsymbol{U}-\boldsymbol{u}^{\infty}(\boldsymbol{x}) \\
\boldsymbol{\Omega}-\frac{1}{2} \boldsymbol{\nabla} \times \boldsymbol{u}^{\infty}(\boldsymbol{x}) \\
\boldsymbol{E}-\boldsymbol{e}^{\infty}(\boldsymbol{x}) \\
\vdots
\end{array}\right)=-\left(\begin{array}{cccc}
\boldsymbol{M}_{U F} & \boldsymbol{M}_{U L} & \boldsymbol{M}_{U S} & \cdots \\
\boldsymbol{M}_{\Omega F} & \boldsymbol{M}_{\Omega L} & \boldsymbol{M}_{\Omega S} & \ldots \\
\boldsymbol{M}_{E F} & \boldsymbol{M}_{E L} & \boldsymbol{M}_{E S} & \ldots \\
\vdots & \vdots & \vdots & \ddots
\end{array}\right) \cdot\left(\begin{array}{c}
\boldsymbol{F}^{H} \\
\boldsymbol{L}^{H} \\
\boldsymbol{S}^{H} \\
\vdots
\end{array}\right),
$$

where $\boldsymbol{u}^{\infty}(\boldsymbol{x})$ is an ambient velocity field that is present in the absence of the particles and $\boldsymbol{e}^{\infty}(\boldsymbol{x})$ is the rate of deformation of the ambient field. Of course, for rigid particles, $\boldsymbol{E}$ (the particle rate of strain) is identically zero. Here, the translational velocities of and forces on $N$ particles are represented in terms of $3 N$-dimensional velocity and force vectors. The grand mobility tensor, often denoted $\mathscr{M}$, is symmetric and positive definite. This is an essential aspect of Stokes flow and is a necessary component of any model of Brownian motion. Additionally, as the grand mobility tensor is linearly coupled to the boundary data, it can only depend on the geometry of the system under study. This includes not only the configuration of the particles, but also the relative position of any boundaries confining the suspension.

Consider the velocity field generated by a suspension of rigid, impenetrable, no-slip particles in an arbitrary bounding geometry. The boundary integral formulation for Stokes flow can be written as (Ladyzhenskaya 1963)

$$
\begin{aligned}
& \boldsymbol{u}(\boldsymbol{x})-\boldsymbol{u}^{\infty}(\boldsymbol{x})=-\sum_{i=1}^{N} \int_{S_{i}} \boldsymbol{G}\left(\boldsymbol{x} ; \boldsymbol{x}^{\prime}\right) \cdot \boldsymbol{\sigma}\left(\boldsymbol{x}^{\prime}\right) \cdot \boldsymbol{n}^{\prime} \mathrm{d} S_{x^{\prime}} \\
& -\int_{S_{\infty}}\left\{\boldsymbol{G}\left(\boldsymbol{x}, \boldsymbol{x}^{\prime}\right) \cdot \sigma\left(\boldsymbol{x}^{\prime}\right) \cdot \boldsymbol{n}^{\prime}-\left[\boldsymbol{u}\left(\boldsymbol{x}^{\prime}\right)-\boldsymbol{u}^{\infty}\left(\boldsymbol{x}^{\prime}\right)\right] \cdot \boldsymbol{\Sigma}\left(\boldsymbol{x}, \boldsymbol{x}^{\prime}\right) \cdot \boldsymbol{n}^{\prime}\right\} \mathrm{d} S_{\boldsymbol{x}^{\prime}},
\end{aligned}
$$

where $\boldsymbol{\sigma}(\boldsymbol{x})$ is the Cauchy stress in the fluid, $\boldsymbol{n}^{\prime}$ is the normal to the surface pointing into the fluid, $\boldsymbol{G}\left(\boldsymbol{x}, \boldsymbol{x}^{\prime}\right)$ is the Green's function for the velocity field in Stokes flow subject to the bounding geometry with field point at $\boldsymbol{x}$ and source at $\boldsymbol{x}^{\prime}$, and

$$
\Sigma_{i j k}\left(\boldsymbol{x}, \boldsymbol{x}^{\prime}\right)=-\delta_{i j} P_{k}\left(\boldsymbol{x}, \boldsymbol{x}^{\prime}\right)+\eta\left[\partial_{x_{i}^{\prime}} G_{j k}\left(\boldsymbol{x}, \boldsymbol{x}^{\prime}\right)+\partial_{x_{j}^{\prime}} G_{i k}\left(\boldsymbol{x}, \boldsymbol{x}^{\prime}\right)\right],
$$

corresponding to the stress field associated with the point force with $\boldsymbol{P}\left(\boldsymbol{x}, \boldsymbol{x}^{\prime}\right)$, the Green's function for the pressure field in Stokes flow, and $\delta_{i j}$ is the Kronecker deltafunction. The $S_{i}$ are the surfaces of the particles, while $S_{\infty}$ is an arbitrary surface that follows the confinement and also bounds a finite portion of the suspension. This forms the basis for the development of the grand mobility tensor of a typical colloidal dispersion, for which a schematic is shown in figure 1 .

We focus on a single particle $n$ for the moment. The velocity field in the fluid may be expressed as

$$
\boldsymbol{u}(\boldsymbol{x})-\boldsymbol{u}^{\infty}(\boldsymbol{x})=-\int_{S_{n}} \boldsymbol{G}\left(\boldsymbol{x}, \boldsymbol{x}^{\prime}\right) \cdot \boldsymbol{\sigma}\left(\boldsymbol{x}^{\prime}\right) \cdot \boldsymbol{n}^{\prime} \mathrm{d} S_{x^{\prime}}+\boldsymbol{u}_{n}^{\prime}(\boldsymbol{x}),
$$

where $\boldsymbol{u}_{n}^{\prime}(\boldsymbol{x})$ is the velocity field generated by the direct forcing and response of other particles and the channel walls. Recasting the problem in this form has no effect on the generality of the flow field, but it allows us to apply directly Faxén's formulae. By integrating (2.4) over the surface of the spherical particle $n$, one can show that the 




FIGURE 1. (Colour online available at journals.cambridge.org/flm) Particles and fluid confined by an arbitrary geometry. The surface of the particles and the surface at infinity are indicated as $S_{i}$ and $S_{\infty}$. Note that the surface at infinity does not cut through the particles.

velocity of particle $n$ is

$$
\begin{aligned}
\boldsymbol{U}_{n}= & -\left(\boldsymbol{M}_{U F}^{(S)} \cdot \boldsymbol{F}_{n}+\boldsymbol{M}_{U L}^{(S)} \cdot \boldsymbol{L}_{n}+\boldsymbol{M}_{U S}^{(S)}: \boldsymbol{S}_{n}+\cdots\right) \\
& +\left.\left(1+\frac{a_{n}^{2}}{6} \nabla_{x}^{2}\right)\left[\boldsymbol{u}^{\infty}(\boldsymbol{x})+\boldsymbol{u}_{n}^{\prime}(\boldsymbol{x})\right]\right|_{\boldsymbol{x}_{n}},
\end{aligned}
$$

where the $\boldsymbol{M}_{\Omega A}^{(S)}$ are elements of the grand mobility tensor for a single particle in the bounding geometry (hence the superscript ' $S$ '), $a_{n}$ is the radius of particle $n$, and $\boldsymbol{x}_{n}$ denotes its centre. Analogous expressions for the torque and rate of deformation arise from integrating the product of the total velocity field and $\boldsymbol{x}-\boldsymbol{x}_{n}$, viz.

$$
\begin{aligned}
\boldsymbol{\Omega}_{n}= & -\left(\boldsymbol{M}_{\Omega F}^{(S)} \cdot \boldsymbol{F}_{n}+\boldsymbol{M}_{\Omega L}^{(S)} \cdot \boldsymbol{L}_{n}+\boldsymbol{M}_{\Omega S}^{(S)}: \boldsymbol{S}_{n}+\cdots\right) \\
& +\frac{1}{2} \nabla \times\left.\left[\boldsymbol{u}^{\infty}(\boldsymbol{x})+\boldsymbol{u}_{n}^{\prime}(\boldsymbol{x})\right]\right|_{\boldsymbol{x}_{n}}, \\
0= & -\left(\boldsymbol{M}_{E F}^{(S)} \cdot \boldsymbol{F}_{n}+\boldsymbol{M}_{E L}^{(S)} \cdot \boldsymbol{L}_{n}+\boldsymbol{M}_{E S}^{(S)}: S_{n}+\cdots\right) \\
& +\left.\left(1+\frac{a_{\alpha}^{2}}{10} \nabla^{2}\right)\left[\boldsymbol{e}^{\infty}(\boldsymbol{x})+\boldsymbol{e}_{n}^{\prime}(\boldsymbol{x})\right]\right|_{\boldsymbol{x}_{n}}
\end{aligned}
$$

where $\boldsymbol{e}_{n}^{\prime}(\boldsymbol{x})$ is the rate of strain of the disturbance velocity field.

For parallel walls, a detailed and computationally friendly method for computing the grand mobility tensor for a single particle is presented in Swan \& Brady (2010). The rest of this paper is focused primarily on the computation of $\boldsymbol{u}_{n}^{\prime}(\boldsymbol{x})$, which is often referred to as the 'disturbance' velocity field. Any model substituted for this quantity must yield a convergent sum of the integrals over the surfaces of the particles and the integral over the surface at infinity. Glendinning \& Russel (1982) introduced the mean stress of the suspension into this formulation and found that, indeed, the divergent hydrodynamic disturbances due to the particles and the integral over the surface at infinity together yield a convergent sum for an unbounded suspension. The more straightforward route inspired by Hasimoto (1959) is taken and this disturbance velocity field is computed directly in the next section.

\subsection{Stokes flow in a channel subject to an arbitrary, periodic body force}

A periodic body force, $\boldsymbol{f}(\boldsymbol{x})$, sets a viscous, incompressible fluid in motion. As the body force has periods $L_{1}$ and $L_{2}$ in the $\boldsymbol{e}_{1}$ and $\boldsymbol{e}_{2}$ directions, the fluid velocity and pressure fields, denoted by $\boldsymbol{u}(\boldsymbol{x})$ and $p(\boldsymbol{x})$, are similarly periodic (see figure 2). Two additional constraints are imposed such that the velocity field is zero at the channel boundaries $\left(x_{3}=0\right.$ and $\left.x_{3}=L_{3}\right)$, though a shear flow is easily introduced through 


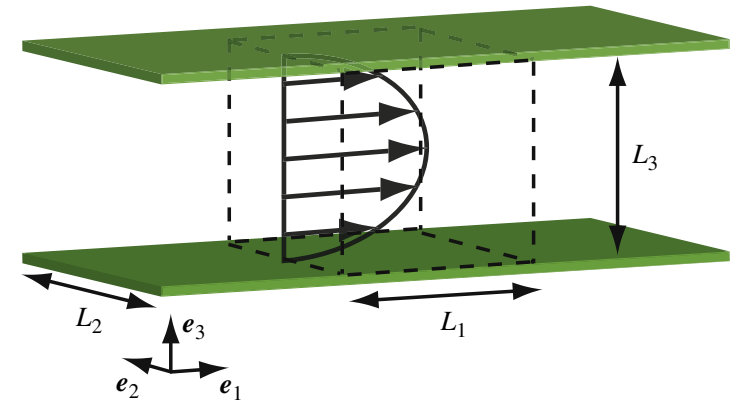

FIgURE 2. (Colour online) The channel geometry with periodic, fictitious streamlines is shown along with the characteristic channel and periodic cell dimensions.

superposition. The Stokes equations govern the values of these dynamic quantities. Each of these periodic variables is written as a Fourier series, and the equations dictating the coefficients of the Fourier series are written as

$$
\begin{aligned}
\boldsymbol{f}(\boldsymbol{x}) & =\sum_{k_{1}, k_{2}} \mathrm{e}^{-2 \pi \mathrm{i} k_{\alpha} x_{\alpha}} \boldsymbol{f}^{(k)}\left(x_{3}\right), \\
\eta \boldsymbol{u}(\boldsymbol{x}) & =\sum_{k_{1}, k_{2}} \mathrm{e}^{-2 \pi \mathrm{i} k_{\alpha} x_{\alpha}} \boldsymbol{u}^{(k)}\left(x_{3}\right), \\
p(\boldsymbol{x}) & =\sum_{k_{1}, k_{2}} \mathrm{e}^{-2 \pi \mathrm{i} k_{\alpha} x_{\alpha}} p^{(k)}\left(x_{3}\right)
\end{aligned}
$$

and

$$
\begin{gathered}
-(2 \pi k)^{2} \boldsymbol{u}^{(k)}+\frac{\partial^{2} \boldsymbol{u}^{(k)}}{\partial x_{3}^{2}}=-2 \pi \mathrm{i} k_{\alpha} \boldsymbol{e}_{\alpha} p^{(k)}+\boldsymbol{e}_{3} \frac{\partial p^{(k)}}{\partial x_{3}}-\boldsymbol{f}^{(k)}, \\
-2 \pi \mathrm{i} k_{\alpha} u_{\alpha}^{(k)}+\frac{\partial u_{3}^{(k)}}{\partial x_{3}}=0,
\end{gathered}
$$

where $k^{2}=k_{\alpha} k_{\alpha}$ and repeated Greek indices signify summation over the index values $(1,2)$. The $\boldsymbol{k}$ are the so-called reciprocal lattice vectors defining the periodic geometry. For a rectilinear lattice, the wavevectors are simply $k_{j}=i / L_{j}$ for $i=0, \pm 1, \pm 2$, etc., while for more complicated lattices, the wavevectors must satisfy the condition that $\boldsymbol{e}_{3} \times \boldsymbol{y}_{i} / L_{1} L_{2}=\boldsymbol{k}_{i}$, where $\boldsymbol{y}_{i}$ is one of two lattice coordinates for which the velocity and pressure are invariant after an integer superposition (e.g. $\boldsymbol{x}+i \boldsymbol{y}_{1}+j \boldsymbol{y}_{2}$ ). The no-slip condition at the channel walls implies that $\boldsymbol{u}^{(k)}(0)=\boldsymbol{u}^{(k)}\left(L_{3}\right)=0$. The solution to these equations can be written as

$$
\begin{aligned}
\boldsymbol{u}^{(k)}\left(x_{3}\right)= & \left(\boldsymbol{I}-4 \pi k x_{3} \boldsymbol{m} \boldsymbol{m}\right) \cdot \boldsymbol{A}^{(k)} \mathrm{e}^{2 \pi k x_{3}} \\
& -\left(\boldsymbol{I}+4 \pi k x_{3} \hat{\boldsymbol{m}} \hat{\boldsymbol{m}}\right) \cdot \boldsymbol{A}^{(k)} \mathrm{e}^{-2 \pi k x_{3}}+\boldsymbol{U}^{(k)}\left(x_{3}\right)
\end{aligned}
$$

and

$$
p^{(k)}\left(x_{3}\right)=-4 \sqrt{2} \pi k\left(\boldsymbol{m} \mathrm{e}^{2 \pi k x_{3}}+\hat{\boldsymbol{m}} \mathrm{e}^{-2 \pi k x_{3}}\right) \cdot \boldsymbol{A}^{(k)}+P^{(k)}\left(x_{3}\right),
$$

where $\boldsymbol{m}=(2 \sqrt{2} \pi k)^{-1}\left(-2 \pi \mathrm{i} k_{\alpha} \boldsymbol{e}_{\alpha}+2 \pi k \boldsymbol{e}_{3}\right), \hat{\boldsymbol{m}}$ is the complex conjugate of $\boldsymbol{m}, \boldsymbol{A}^{(k)}$ is a coefficient to be determined by the boundary conditions, and $\boldsymbol{U}^{(k)}\left(x_{3}\right)$ and $P^{(k)}\left(x_{3}\right)$ are the particular solutions to the Stokes equations. These are written as convolutions of 
the body force with the homogeneous part of the solution such that

$$
\boldsymbol{U}^{(k)}\left(x_{3}\right)=\left[\boldsymbol{I}-\frac{1}{2}(\boldsymbol{m} \hat{\boldsymbol{m}}+\hat{\boldsymbol{m}} \boldsymbol{m})\right] \cdot \boldsymbol{C}_{1}^{(k)}\left(x_{3}\right)+\boldsymbol{m} \boldsymbol{m} \cdot \boldsymbol{C}_{2}^{(k)}\left(x_{3}\right)+\hat{\boldsymbol{m}} \hat{\boldsymbol{m}} \cdot \boldsymbol{C}_{3}^{(k)}\left(x_{3}\right),(2
$$

where

$$
\begin{gathered}
\boldsymbol{C}_{1}^{(k)}\left(x_{3}\right)=-\frac{1}{2 \pi k} \int_{0}^{x_{3}} \sinh \left[2 \pi k\left(x_{3}-x_{3}^{\prime}\right)\right] \boldsymbol{f}^{(k)}\left(x_{3}^{\prime}\right) \mathrm{d} x_{3}^{\prime}, \\
\boldsymbol{C}_{2}^{(k)}\left(x_{3}\right)=\frac{1}{2} \int_{0}^{x_{3}}\left(x_{3}-x_{3}^{\prime}\right) \mathrm{e}^{2 \pi k\left(x_{3}-x_{3}^{\prime}\right)} \boldsymbol{f}^{(k)}\left(x_{3}^{\prime}\right) \mathrm{d} x_{3}^{\prime}, \\
\boldsymbol{C}_{3}^{(k)}\left(x_{3}\right)=\frac{1}{2} \int_{0}^{x_{3}}\left(x_{3}-x_{3}^{\prime}\right) \mathrm{e}^{-2 \pi k\left(x_{3}-x_{3}^{\prime}\right)} \boldsymbol{f}^{(k)}\left(x_{3}^{\prime}\right) \mathrm{d} x_{3}^{\prime}
\end{gathered}
$$

and

$$
P^{(k)}\left(x_{3}\right)=\sqrt{2}\left[\boldsymbol{m} \cdot\left(\boldsymbol{C}_{2}^{\prime}\left(x_{3}\right)-2 \pi k \boldsymbol{C}_{2}\left(x_{3}\right)\right)+\hat{\boldsymbol{m}} \cdot\left(\boldsymbol{C}_{3}^{\prime}\left(x_{3}\right)+2 \pi k \boldsymbol{C}_{3}\left(x_{3}\right)\right)\right] .
$$

These equations satisfy the boundary condition on the lower wall by construction. The three integrals, $\boldsymbol{C}_{i}^{(k)}\left(x_{3}\right)$, represent the combined (2.16) and separate (2.17) and (2.18) effects of exponential decay from the lower and upper channel walls, respectively. The vectors $\boldsymbol{m}$ and $\hat{\boldsymbol{m}}$ reflect the natural coordinate system for the flow field, as each corresponds to gradients of a Fourier summation modulated by exponential growth and decay, respectively. Applying the no-slip condition on the upper wall resolves the remaining unknown coefficient, $\boldsymbol{A}^{(k)}$, viz.

$$
\begin{aligned}
& \boldsymbol{m} \cdot \boldsymbol{A}^{(k)}= {\left[1+\frac{1}{2}\left(4 \pi k L_{3}\right)^{2}-\cosh \left(4 \pi k L_{3}\right)\right]^{-1} } \\
& \times\left[\sinh \left(2 \pi k L_{3}\right) \boldsymbol{m}+2 \pi k L_{3} \mathrm{e}^{-2 \pi k L_{3}} \hat{\boldsymbol{m}}\right] \cdot \boldsymbol{U}^{(k)}\left(L_{3}\right), \\
& \hat{\boldsymbol{m}} \cdot \boldsymbol{A}^{(k)}= {\left[1+\frac{1}{2}\left(4 \pi k L_{3}\right)^{2}-\cosh \left(4 \pi k L_{3}\right)\right]^{-1} } \\
& \times\left[2 \pi k L_{3} \mathrm{e}^{2 \pi k L_{3}} \boldsymbol{m}+\sinh \left(2 \pi k L_{3}\right) \hat{\boldsymbol{m}}\right] \cdot \boldsymbol{U}^{(k)}\left(L_{3}\right), \\
& \boldsymbol{A}^{(k)}=-\frac{1}{2} \operatorname{csch}\left(2 \pi k L_{3}\right)\left[\boldsymbol{U}^{(k)}\left(L_{3}\right)-4 \pi k L_{3}\left(\mathrm{e}^{2 \pi k L_{3}} \boldsymbol{m} \boldsymbol{m} \cdot \boldsymbol{A}^{(k)}+\mathrm{e}^{-2 \pi k L_{3}} \hat{\boldsymbol{m}} \hat{\boldsymbol{m}} \cdot \boldsymbol{A}^{(k)}\right)\right] .
\end{aligned}
$$

However, these formulae for the velocity field and pressure Fourier coefficients only apply when the total wavevector, $k$, is greater than zero. In the zero-wavevector limit, the Fourier coefficients of the velocity field and pressure are

$$
\begin{gathered}
\boldsymbol{u}^{(0)}\left(x_{3}\right)=\left(\boldsymbol{I}-\boldsymbol{e}_{3} \boldsymbol{e}_{3}\right) \cdot\left[\frac{1}{2} x_{3}\left(x_{3}-L_{3}\right) \boldsymbol{g}+\frac{x_{3}}{L_{3}} \int_{0}^{L_{3}} \int_{0}^{x_{3}^{\prime}} \boldsymbol{f}^{(0)}\left(x_{3}^{\prime \prime}\right) \mathrm{d} x_{3}^{\prime \prime} \mathrm{d} x_{3}^{\prime}\right. \\
\left.-\int_{0}^{x_{3}} \int_{0}^{x_{3}^{\prime}} \boldsymbol{f}^{(0)}\left(x_{3}^{\prime \prime}\right) \mathrm{d} x_{3}^{\prime \prime} \mathrm{d} x_{3}^{\prime}\right], \\
p^{(0)}\left(x_{3}\right)=A^{(0)} \cdot \boldsymbol{x}+\boldsymbol{e}_{3} \cdot \int_{0}^{x_{3}} \boldsymbol{f}^{(0)}\left(x_{3}^{\prime}\right) \mathrm{d} x_{3}^{\prime},
\end{gathered}
$$

where $\boldsymbol{g}$ is a macroscopic pressure gradient, and we have reinterpreted $p^{(k)}\left(x_{3}\right)$ as the Fourier transformation of $p-\boldsymbol{g} \cdot\left(\boldsymbol{I}-\boldsymbol{e}_{3} \boldsymbol{e}_{3}\right) \cdot \boldsymbol{x}$, the difference between the total pressure and the macroscopic gradient. A condition setting the value of the constant pressure gradient is required. In this case, the ends of the channel may be left open such that $g$ is prescribed (i.e. drives a Poiseuille flow), or the ends of the channel may be closed so that there is no net flow, viz.

$$
\left(\boldsymbol{I}-\boldsymbol{e}_{3} \boldsymbol{e}_{3}\right) \cdot \int_{0}^{L_{3}} \boldsymbol{u}^{(0)}\left(x_{3}\right) \mathrm{d} x_{3}=0 .
$$


The former condition is somewhat different from unbounded Stokes flow, whereas in the latter condition, conservation of mass manifests itself as the back-flow pressure gradient. In that case (no net flow), the back-flow pressure is

$$
\begin{aligned}
\boldsymbol{g}= & \frac{12}{L_{3}^{2}}\left(\boldsymbol{I}-\boldsymbol{e}_{3} \boldsymbol{e}_{3}\right) \cdot\left[\frac{1}{2} \int_{0}^{L_{3}} \int_{0}^{x_{3}^{\prime}} \boldsymbol{f}^{(0)}\left(x_{3}^{\prime \prime}\right) \mathrm{d} x_{3}^{\prime \prime} \mathrm{d} x_{3}^{\prime}\right. \\
& \left.-\frac{1}{L_{3}} \int_{0}^{L_{3}} \int_{0}^{x_{3}} \int_{0}^{x_{3}^{\prime}} \boldsymbol{f}^{(0)}\left(x_{3}^{\prime \prime}\right) \mathrm{d} x_{3}^{\prime \prime} \mathrm{d} x_{3}^{\prime} \mathrm{d} x_{3}\right] .
\end{aligned}
$$

Indeed, both of these conditions still conserve the momentum of the entire system (particles, fluid and walls). The stresses on the upper and lower walls are periodic in the same fashion as the body force, pressure and velocity field. When the Fourier coefficients of the stress in the fluid are denoted as $\sigma^{(k)}\left(x_{3}\right)$, the total force on the channel walls over one period is

$$
\boldsymbol{F}_{w}=\boldsymbol{e}_{3} \cdot\left(\boldsymbol{\sigma}^{(0)}(0)-\boldsymbol{\sigma}^{(0)}\left(L_{3}\right)\right)=-\int_{0}^{L_{3}} \boldsymbol{f}^{(0)}\left(x_{3}^{\prime}\right) \mathrm{d} x_{3}^{\prime}+L_{3} \boldsymbol{g} .
$$

It is apparent that when the channel walls are left open, this is essentially a statement of Newtonian mechanics, since the force on the walls must be equal and opposite to the total force applied to the fluid. The zero-wavevector contributions to the pressure and velocity fulfil this imperative. Even for arbitrary values of $\boldsymbol{g}$, one finds that the additional stress in the fluid due to the superimposed Poiseuille flow is transmitted to the walls. In the case of a closed channel, the pressure gradient $\boldsymbol{g}$ assumes a very particular form, however. In this case, the sealed ends of the channel bear some of the load due to the particles as well. This reduces the stress on the walls by the amount given exactly by $L_{3} g$.

Note that, while the prescribed boundary condition on the walls was no-slip with the walls unmoving, a shear flow in the channel may be generated by moving one wall relative to the other at constant speed. No alteration in the methodology is needed, as this shear flow can be freely superimposed with the flow field as already derived. With the substitution of an appropriate, periodic body force, these expressions completely define the pressure and velocity fields in a no-slip channel. For these purposes, the body force will be that due to a dispersion of colloidal particles suspended in the fluid. However, this solution could find equal applicability in studying electro-osmotic flow in the weak field limit or the flow generated in certain microfluidic devices. Appendix A treats the case of rigid, impenetrable, shear-stress-free walls, which might model a viscous fluid bound between two inviscid layers with planar interfaces maintained by a sufficiently large surface tension. While an algorithm for slipping channels is not developed any further, every step described from here on is just as suitable to that particular case.

\subsection{The Ewald summation technique}

The velocity field generated by a three-dimensionally periodic body force in a viscous fluid is

$$
\eta \boldsymbol{u}(\boldsymbol{x})=\sum_{\xi \neq 0} \mathrm{e}^{-2 \pi \mathrm{i} \xi \cdot \boldsymbol{x}}(2 \pi \xi)^{-2}(\boldsymbol{I}-\hat{\boldsymbol{\xi}} \hat{\xi}) \cdot \boldsymbol{f}^{(\xi)},
$$

where the $\xi$ are the three-dimensional reciprocal lattice vectors and the $f^{(\xi)}$ term represents the three-dimensional Fourier coefficients of the body force. As Hasimoto showed, for even the simplest body force (an array of point forces), this summation 
is slowly converging. The standard technique for accelerating the convergence of solutions subject to Poisson-like equations is referred to as the Ewald summation method. Rather than detail the entire calculation in general, the result for the preceding hydrodynamic case is shown and then expanded. The Ewald summation form of (2.28) is

$$
\begin{aligned}
\eta \boldsymbol{u}(\boldsymbol{x})= & \frac{1}{4 \pi} \int_{V}\left[\frac{\phi_{-1 / 2}\left(\pi r^{2} / \alpha\right)}{\alpha^{1 / 2}} \boldsymbol{I}-\frac{\pi r^{2} \phi_{1 / 2}\left(\pi r^{2} / \alpha\right)}{\alpha^{3 / 2}}(\boldsymbol{I}-\hat{\boldsymbol{r}} \hat{\boldsymbol{r}})\right] \cdot \boldsymbol{f}\left(\boldsymbol{x}^{\prime}\right) \mathrm{d} \boldsymbol{x}^{\prime} \\
& +\sum_{\xi \neq 0} \mathrm{e}^{-2 \pi \mathrm{i} \cdot \boldsymbol{x}}(2 \pi \xi)^{-2}\left(1+\pi \alpha \xi^{2}\right) \mathrm{e}^{-\pi \alpha \xi^{2}}(\boldsymbol{I}-\hat{\boldsymbol{\xi}} \hat{\boldsymbol{\xi}}) \cdot \boldsymbol{f}^{(\xi)},
\end{aligned}
$$

where $\boldsymbol{r}=\boldsymbol{x}-\boldsymbol{x}^{\prime}, r^{2}=\boldsymbol{r} \cdot \boldsymbol{r}$ and $\alpha$ is the so-called splitting parameter. For a splitting parameter of appropriate magnitude, both the integral and the Fourier summation are rapidly convergent. The function $\phi_{\nu}$ is the incomplete $\Gamma$-function and is represented as the integral

$$
\phi_{\nu}(y)=\int_{1}^{\infty} \psi^{\nu} \mathrm{e}^{-y \psi} \mathrm{d} \psi .
$$

While the details of this calculation are hardly trivial and difficult to apply to the two-dimensional geometry, inspiration is drawn from it in order to achieve a rapidly converging solution to the problem of fluid motion in a channel. Notice that the summation in (2.29) is the exact solution for a velocity field subject to a force with Fourier coefficients $\left(1+\pi \alpha \xi^{2}\right) \exp \left(-\pi \alpha \xi^{2}\right) \boldsymbol{f}^{(\xi)}$. Thus, the key to ensuring rapid convergence in Stokes flow is modulating the force density by a Gaussian. This body force is denoted as $\boldsymbol{f}_{g}(\boldsymbol{x})$ - the 'global' body force - since it contains the long-range effects on the fluid. Conversely $f(x)-f_{g}(x)$ encompasses the body force giving rise to short-range effects on the fluid. The two-dimensional Fourier coefficients of $\boldsymbol{f}_{g}(\boldsymbol{x})$ are found directly via the convolution theorem for Fourier transformations, viz.

$$
\begin{aligned}
\boldsymbol{f}_{g}^{(k)}\left(x_{3}\right)= & \frac{1}{2 \alpha^{1 / 2}} \int_{-\infty}^{\infty}\left(3+2 \pi \alpha k^{2}-\frac{2 \pi x_{3}^{\prime 2}}{\alpha}\right) \\
& \times \mathrm{e}^{-\left[\pi \alpha k^{2}+\left(\pi x_{3}^{\prime 2} / \alpha\right)\right]} \boldsymbol{f}^{(k)}\left(x_{3}-x_{3}^{\prime}\right) \mathrm{d} x_{3}^{\prime} .
\end{aligned}
$$

Similarly, the global force contribution itself is

$$
\boldsymbol{f}_{g}(\boldsymbol{x})=\frac{1}{\alpha^{3 / 2}} \int_{V}\left(\frac{5}{2}-\frac{\pi r^{2}}{\alpha}\right) \mathrm{e}^{-\left(\pi r^{2} / \alpha\right)} \boldsymbol{f}\left(\boldsymbol{x}^{\prime}\right) \mathrm{d} \boldsymbol{x}^{\prime},
$$

where once again $\boldsymbol{r}=\boldsymbol{x}-\boldsymbol{x}^{\prime}$ and $r^{2}=\boldsymbol{r} \cdot \boldsymbol{r}$. This is identical to the force density that Hernández-Ortiz et al. (2007) found 'convenient' for their Ewald-like simulation method, in that it produces a simple, closed-form pressure and velocity field. This body force is not only convenient but also a direct consequence of the Ewald summation technique in an unbounded geometry. Substituting $\boldsymbol{f}_{g}^{(k)}\left(x_{3}\right)$ into (2.8)-(2.24) yields a rapidly converging summation describing the long-range effects of the body force and boundaries on the fluid, which is denoted $\boldsymbol{u}_{g}(\boldsymbol{x})$ - the 'global' velocity field. What remains is to solve the problem

$$
\begin{gathered}
\eta \nabla^{2} \boldsymbol{u}_{l}=\nabla p_{l}-\boldsymbol{f}(\boldsymbol{x})+\boldsymbol{f}_{g}(\boldsymbol{x}), \\
\boldsymbol{\nabla} \cdot \boldsymbol{u}_{l}=0,
\end{gathered}
$$

describing the local fluid motion $\left(\boldsymbol{u}_{l}(\boldsymbol{x})\right)$ and local pressure $\left(p_{l}(\boldsymbol{x})\right)$ due to the rapidly decaying modes of the body force. In this way, the velocity field generated by a body 
force in a channel may be represented as a one-to-one superposition of $\boldsymbol{u}_{g}(\boldsymbol{x})$ and $\boldsymbol{u}_{l}(\boldsymbol{x})$.

\subsection{Reflections in real space}

The local body force $\left(\boldsymbol{f}(\boldsymbol{x})-\boldsymbol{f}_{g}(\boldsymbol{x})\right)$ yields a velocity field in an unbounded fluid that decays exponentially fast with respect to both $\alpha$ and $x$. However, since this is a confined geometry, the interplay of body force and no-slip condition on the walls must be explored further before deciding on an appropriate approximation of $\boldsymbol{u}_{l}(\boldsymbol{x})$. One method of representing the Stokes flow between two channel walls is through the use of the method of reflections. This method treats the material outside the channel walls as fluid subject to an appropriate 'image' body force. Often, the channel walls are treated as independent and the fluid flow is represented as a superposition of the flow above and below the half-space on either side of the channel such that the combined flow results in a no-slip condition at that single wall only. Take as an example a Stokeslet

$$
\boldsymbol{J}(\boldsymbol{r})=(8 \pi \eta r)^{-1}(\boldsymbol{I}+\hat{\boldsymbol{r}} \hat{\boldsymbol{r}})
$$

located at $\boldsymbol{x}^{\prime}$ and a distance $x_{3}^{\prime}$ above a no-slip wall. Blake (1971) determined the reflected flow field that preserves a no-slip condition at $x_{3}=0$, viz.

$$
\begin{aligned}
\boldsymbol{J}_{w}\left(\boldsymbol{R}, x_{3}^{\prime}\right)= & -\boldsymbol{J}(\boldsymbol{R})+x_{3}^{\prime 2} \nabla_{x}^{2} \boldsymbol{J}(\boldsymbol{R}) \cdot\left(\boldsymbol{I}-2 \boldsymbol{e}_{3} \boldsymbol{e}_{3}\right) \\
& -2 x_{3}^{\prime}\left[\left(\boldsymbol{I}-2 \boldsymbol{e}_{3} \boldsymbol{e}_{3}\right) \cdot \nabla_{x} \boldsymbol{J}(\boldsymbol{R}) \cdot \boldsymbol{e}_{3}\right]^{\mathrm{T}},
\end{aligned}
$$

where $\boldsymbol{R}=\boldsymbol{r}+2 x_{3}^{\prime} \boldsymbol{e}_{3}$ and the superscript $T$ indicates transposition. While the Stokeslet decays as $r^{-1}$, the reflected flow decays as $R^{-1}$. That is, the reflected flow appears as a source located a significant distance from the original point force. If the second channel wall is reintroduced and the subsequent reflections to satisfy the no-slip condition at $x_{3}=L_{3}$ calculated, one finds that they act as sources located even further away from the initial point force. Liron \& Mochon (1976) showed that the decay of the subsequent reflections is at its slowest, algebraic, and at its fastest, exponential. Since the local velocity field already decays as a Gaussian, only one reflection of the body force is considered as higher-order reflections will decay at more than twice the exponential rate. To that end, the Stokeslet and its reflection are invoked as the Green's functions for Stokes flow above a no-slip wall such that the locally reflected velocity field is

$$
\begin{aligned}
\boldsymbol{u}_{l}(\boldsymbol{x})= & \int_{V}\left[\boldsymbol{J}(\boldsymbol{r})+\boldsymbol{J}_{w}\left(\boldsymbol{R}, x_{3}^{\prime}\right)\right] \\
& \times\left\{\int_{V}\left[\delta\left(\boldsymbol{r}^{\prime}\right)-\frac{1}{\alpha^{3 / 2}}\left(\frac{5}{2}-\frac{\pi r^{\prime 2}}{\alpha}\right) \mathrm{e}^{-\left(\pi r^{2} / \alpha\right)}\right] \boldsymbol{f}\left(\boldsymbol{x}^{\prime \prime}\right) \mathrm{d} \boldsymbol{x}^{\prime \prime}\right\} \mathrm{d} \boldsymbol{x}^{\prime},
\end{aligned}
$$

where $\boldsymbol{r}^{\prime}=\boldsymbol{x}^{\prime}-\boldsymbol{x}^{\prime \prime}$ and $\boldsymbol{r}^{2}=\boldsymbol{r}^{\prime} \cdot \boldsymbol{r}^{\prime}$ and the term in braces is the local force density. Because the Stokes equations are unique, switching the order of integration above demonstrates that the Stokeslet contribution to the integral in (2.37) corresponds directly to the real-space integrand in (2.29), viz.

$$
\begin{aligned}
\boldsymbol{G}\left(\boldsymbol{r}^{\prime \prime} ; \alpha\right) & =\int_{V} \boldsymbol{J}(\boldsymbol{r})\left[\delta\left(\boldsymbol{r}^{\prime}\right)-\frac{1}{\alpha^{3 / 2}}\left(\frac{5}{2}-\frac{\pi r^{\prime 2}}{\alpha}\right) \mathrm{e}^{-\left(\pi r^{\prime 2} / \alpha\right)}\right] \mathrm{d} \boldsymbol{x}^{\prime} \\
& =\frac{1}{4 \pi \eta}\left[\frac{\phi_{-1 / 2}\left(\pi r^{\prime \prime 2} / \alpha\right)}{\alpha^{1 / 2}} \boldsymbol{I}-\frac{\pi r^{\prime \prime 2} \phi_{1 / 2}\left(\pi r^{\prime \prime 2} / \alpha\right)}{\alpha^{3 / 2}}\left(\boldsymbol{I}-\hat{\boldsymbol{r}}^{\prime \prime} \hat{\boldsymbol{r}}^{\prime \prime}\right)\right],
\end{aligned}
$$


where $\boldsymbol{r}^{\prime \prime}=\boldsymbol{x}-\boldsymbol{x}^{\prime \prime}$ and $r^{\prime \prime 2}=\boldsymbol{r}^{\prime \prime} \cdot \boldsymbol{r}^{\prime \prime}$. As the integral in (2.37) is rather difficult to compute, since the $\boldsymbol{r}$ and $\boldsymbol{r}^{\prime}$ directors are not concentric with respect to $\boldsymbol{x}^{\prime}$, this represents a fortuitous simplification, which is useful in finding closed-form expressions for the integrals over $\boldsymbol{J}_{w}\left(\boldsymbol{R}, x_{3}^{\prime}\right)$ and produces an expression for the local velocity field in terms of a single integral over the total force density. The details of this calculation are tedious but explained in Appendix C. The result is quite simple, however,

$$
\boldsymbol{u}_{l}(\boldsymbol{x})=\int_{V}\left[\boldsymbol{G}(\boldsymbol{r} ; \alpha)+\boldsymbol{G}_{w}\left(\boldsymbol{R}, x_{3}^{\prime} ; \alpha\right)\right] \cdot \boldsymbol{f}\left(\boldsymbol{x}^{\prime}\right) \mathrm{d} \boldsymbol{x}^{\prime},
$$

where the tensor, $\boldsymbol{G}_{w}\left(\boldsymbol{R}, x_{3}^{\prime} ; \alpha\right)$, is the reflection of the local velocity field and is provided in Appendix C. With this, the local velocity field including the reflection off the lower wall is completely defined. To find the flow due to the no-slip condition on the upper wall, Blake's reflection is applied again, albeit with a slightly different set of coordinates, viz.

$$
\begin{aligned}
\boldsymbol{J}_{w}\left(\tilde{\boldsymbol{R}}, x_{3}^{\prime}\right)= & -\boldsymbol{J}(\tilde{\boldsymbol{R}})+\left(L_{3}-x_{3}^{\prime}\right)^{2} \nabla_{x}^{2} \boldsymbol{J}(\tilde{\boldsymbol{R}}) \cdot\left(\boldsymbol{I}-2 \boldsymbol{e}_{3} \boldsymbol{e}_{3}\right) \\
& -2\left(L_{3}-x_{3}^{\prime}\right)\left[\left(\boldsymbol{I}-2 \boldsymbol{e}_{3} \boldsymbol{e}_{3}\right) \cdot \nabla_{x} \boldsymbol{J}(\tilde{\boldsymbol{R}}) \cdot \boldsymbol{e}_{3}\right]^{\mathrm{T}},
\end{aligned}
$$

where $\tilde{\boldsymbol{R}}=\boldsymbol{r}-2\left(L_{3}-x_{3}^{\prime}\right) \boldsymbol{e}_{3}$. Precisely the same operations apply to this reflected field as well such that a one-to-one superposition of the reflections due to each wall independently approximates the local contribution to the velocity field in the channel.

Appendix $\mathrm{C}$ demonstrates that the reflected flow decays at its slowest as $\exp \left(-\pi R^{2} / \alpha\right)$, while the flow due to the local velocity field decays like $\exp \left(-\pi r^{2} / \alpha\right)$. For any source point $\left(\boldsymbol{x}^{\prime}\right)$ or field point $(\boldsymbol{x})$ near the wall, the connecting vector and its reflection are similar in magnitude (i.e. $r \approx R$ ), and the reflected flow is just as strong as the unreflected flow itself. In fact, the flow due to local force density alone is insufficient, and the reflected flow must be included to avoid errors, which are of the order of $100 \%$ in the local velocity field - this is illustrated in figure 3 .

This approximation is best for values of the splitting parameter such that $\sqrt{\alpha} \leqslant L_{3}$. Under these circumstances, the reflected flow decays quickly enough that it is essentially unfelt on the opposing wall. However, if the value of $\alpha$ is determined on this basis alone, the wave-space summation may not converge rapidly. This raises an interesting issue, as one of the problems often pursued in this field is the dynamics of suspensions in suitably narrow channels (i.e. $L_{3} \approx$ a few particle radii). In this case, however, the far-field hydrodynamic interactions decay like $\left(r / L_{3}\right)^{-2}$ and the lubrication interactions between the particles and the channel walls dominate the hydrodynamic resistance. Therefore, no lower bound on the splitting parameter is necessary. In the rest of this section, it is shown that the above solution for the velocity field due to a periodic body force constitutes a far-field approximation to the hydrodynamic resistance felt by particles in a suspension. This does not replicate the singular lubrication forces, however. Instead, the Stokesian dynamics method is used to model those interactions explicitly.

\subsection{Computations in wave space}

The calculation must now evolve from the abstract perspective of body forces and fluid velocities to that of force moments on and velocities of particles in suspension. To this 


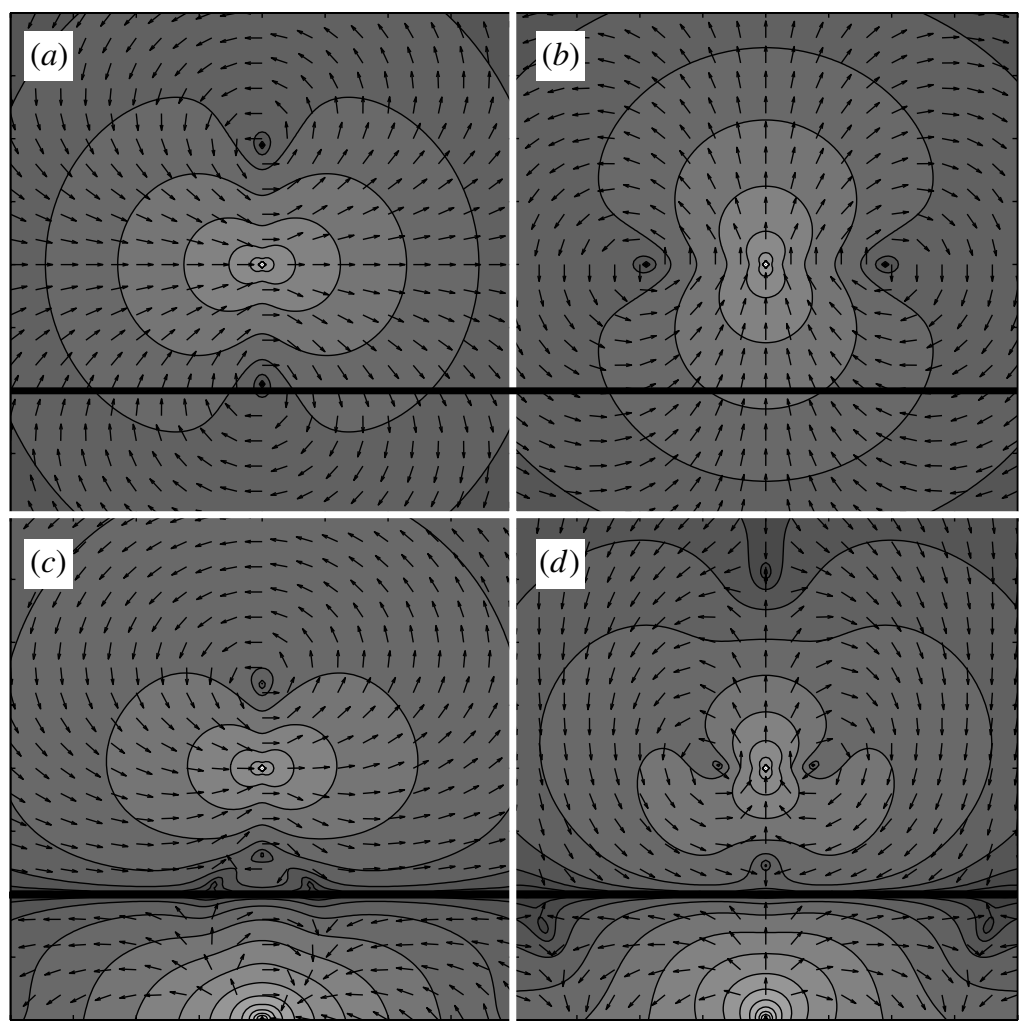

FIGURE 3. The real-space contribution to the velocity field generated by a point force differs significantly when unbounded $(a, b)$ and when near a wall $(c, d)$. In this case, the distance of the source from the wall (indicated by the thick line) is $\sqrt{\alpha / 10}$. The panels depict the flow due to a force parallel to the wall $(a, c)$ and perpendicular to the wall $(b, d)$ as streamlines and contours of the flow magnitude. While the absolute magnitude is inconsequential, the contour lines are logarithmically distributed. The Gaussian decay is evident for the unbounded flows; however, a stronger decay is present for the bounded flow fields.

end, the body force in the fluid is written as

$$
\boldsymbol{f}(\boldsymbol{x})=\sum_{n} \delta\left(\boldsymbol{x}-\boldsymbol{x}_{n}\right) \boldsymbol{F}_{n},
$$

which reflects a periodically replicated system of spherical particles with force $\boldsymbol{F}_{n}$ on the particle with centre at $\boldsymbol{x}_{n}$. Although particles themselves are not adequately represented as point forces, a multipole expansion of the force density on the particle surfaces always yields a linear summation of the particle force moments (e.g. force, torque, stresslet, etc.). Later discussion will explain how higher-order force moments are incorporated into this formulation. For now, focus is restricted to the simpler case of point forces. With this, the global force density becomes

$$
\boldsymbol{f}_{g}^{(k)}\left(x_{3}\right)=\frac{1}{2 \alpha^{1 / 2}} \sum_{n}\left(3+2 \pi \alpha k^{2}-\frac{2 \pi \Delta x_{3}^{(n) 2}}{\alpha}\right) \mathrm{e}^{-\pi \alpha k^{2}-\frac{\pi \Delta x_{3}^{(n) 2}}{\alpha}} \boldsymbol{F}_{n}^{(k)},
$$


where $\Delta x_{3}^{(n)}=x_{3}-x_{3}^{(n)}$ and $\boldsymbol{F}_{n}^{(k)}=\exp \left(2 \pi \mathrm{i} k_{\alpha} x_{\alpha}^{(n)}\right) \boldsymbol{F}_{n}$. Applying this to the solution developed earlier in the section completely defines a rapidly converging Ewald summation for the global contribution to the fluid velocity. Equations (2.16)-(2.18) are calculated in Appendix B using this force density. The key here is the recovery of the essential linearity associated with Stokes flow. The local and global velocity fields, while comprising two different levels of computation are in the end just linear transformations of the forces on the particles.

Multipole expansion allows the incorporation of higher-order force moments into this formulation. Returning to (2.8) and (2.13), recognize that the global contribution to the disturbance velocity due to a suspension of particles can be written as

$$
\boldsymbol{u}_{g}(\boldsymbol{x})=\sum_{n}^{N} \int_{S_{n}} \sum_{k_{1}, k_{2}} \mathrm{e}^{2 \pi \mathrm{i} k_{\alpha} x_{\alpha}} \boldsymbol{G}^{(k)}\left(x_{3}, x_{3}^{\prime}\right) \cdot \sum_{k_{1}^{\prime}, k_{2}^{\prime}} \mathrm{e}^{2 \pi k_{\alpha}^{\prime} x_{\alpha}^{\prime}} \boldsymbol{f}_{g}^{\left(k^{\prime}\right)}\left(x_{3}^{\prime}\right) \mathrm{d} \boldsymbol{x}^{\prime},
$$

where $\boldsymbol{G}^{(k)}\left(\boldsymbol{x}, \boldsymbol{x}^{\prime}\right)$ is simply the Fourier coefficient of the Green's function for Stokes flow in a channel. This is computed using the solution developed in $\S 2.2$ with the substitution of a delta-function for the force density. Because the velocity field is still a solution to the Stokes equations, it is bi-harmonic. Therefore, the typical Taylor expansion of the Green's function about the centre of each particle is used to eliminate the integral, viz.

$$
\begin{aligned}
\boldsymbol{u}_{g}(\boldsymbol{x})= & \sum_{n}^{N}\left[\left(1+\frac{a_{n}^{2}}{6} \nabla_{\boldsymbol{x}^{\prime}}\right) \boldsymbol{F}_{n} \cdot\right. \\
& -\frac{1}{2} \boldsymbol{L}_{n} \cdot \nabla_{\boldsymbol{x}^{\prime}} \times \\
& \left.+\frac{1}{2}\left(\nabla_{\boldsymbol{x}^{\prime}}+\frac{1}{2} \nabla_{\boldsymbol{x}^{\prime}}^{\mathrm{T}}\right)\left(1+\frac{a_{n}^{2}}{10} \nabla_{\boldsymbol{x}^{\prime}}^{2}\right) \cdot \boldsymbol{S}_{n} \cdot+\cdots\right] \sum_{k_{1}, k_{2}} \mathrm{e}^{2 \pi i k_{\alpha} x_{\alpha}^{\prime}} \boldsymbol{G}^{(k) T}\left(x_{3}, x_{3}^{\prime}\right) \\
& \times\left.\left[\frac{1}{2 \alpha^{1 / 2}} \sum_{n}\left(3+2 \pi \alpha k^{2}-\frac{2 \pi \Delta x_{3}^{(n) 2}}{\alpha}\right) \mathrm{e}^{-\pi \alpha k^{2}-\pi \Delta x_{3}^{(n) 2} / \alpha}\right]\right|_{x^{\prime}=x_{n}} .
\end{aligned}
$$

In this way, the disturbance flow generated by particle $n$ can be represented as contributions due to each of the moments of the force density on its surface. Note that the force moments each propagate via an effective Green's function and much care must be taken in evaluating the derivatives with respect to the source point of the flow. This has a far-reaching influence on the particle dynamics (see Swan \& Brady 2007).

\subsection{Simulation methods}

In this section, the Stokesian dynamics method for computing hydrodynamic interactions among many particles is illustrated. Since the mobility and resistance tensors are purely a function of the system geometry, the formal construction of a Stokesian dynamics simulation does not depend on any macroscopic boundary (Brady $\&$ Bossis 1988). The same is true of accelerated Stokesian dynamics. Therefore, the solution for the velocity field in a channel can be used to generate a rapid simulation of many colloidal particles as in the accelerated Stokesian dynamics simulations of Sierou \& Brady (2001). 


\subsubsection{Stokesian dynamics}

Since the Stokes equations governing the fluid physics are linear, the hydrodynamic force on the particles is coupled linearly to the particle velocities such that $\boldsymbol{F}^{H}=-\boldsymbol{R}_{F U} \cdot\left(\boldsymbol{U}-\boldsymbol{u}^{\infty}\right)+\boldsymbol{R}_{F E}: \boldsymbol{E}^{\infty}$, where $\boldsymbol{R}_{F U}$ and $\boldsymbol{R}_{F E}$ are resistance tensors. A notation in which the force and torque are combined into a generalized force vector is imposed here without loss of generality. Clearly, generating and inverting the resistance tensor quickly is the crux of the dynamical problem. To that end, the typical Stokesian dynamics paradigm is followed and the hydrodynamic force is separated into near-field (or lubrication) and far-field contributions, viz.

$$
0=-\boldsymbol{R}_{F U}^{n f} \cdot\left(\boldsymbol{U}-\boldsymbol{u}^{\infty}\right)+\boldsymbol{F}^{H, f f}+\boldsymbol{R}_{F E}^{n f}: \boldsymbol{E}^{\infty}+\boldsymbol{F}^{P}
$$

and

$$
\left(\begin{array}{c}
\boldsymbol{U}-\boldsymbol{u}^{\infty} \\
-\boldsymbol{E}^{\infty}
\end{array}\right)=-\mathscr{M}^{\infty} \cdot\left(\begin{array}{c}
\boldsymbol{F}^{H, f f} \\
\boldsymbol{S}^{H, f f}
\end{array}\right),
$$

where $\boldsymbol{R}_{F U}^{n f}$ is the exact two-body lubrication contribution to the resistance tensor less the two-body far-field contribution, $\boldsymbol{F}^{H . f f}$ is the far-field hydrodynamic force, $\boldsymbol{E}^{\infty}$ is the average rate of strain in the suspension, $\mathscr{M}^{\infty}$ is the far-field mobility tensor and $S^{H, f f}$ is the far-field stresslet. We have included an arbitrary force denoted $\boldsymbol{F}^{P}$, which may represent, for instance, gravity, electrostatic forces or inter-particle forces among the particles. With a few algebraic manipulations, this system of equations can be solved for the far-field hydrodynamic force and the particle velocities as

$$
\begin{aligned}
\left(\begin{array}{c}
\tilde{\boldsymbol{F}}^{H, f f} \\
\boldsymbol{S}^{H, f f}
\end{array}\right)= & {\left[\mathscr{M}^{\infty}+\left(\begin{array}{cc}
\left(\tilde{\boldsymbol{R}}_{F U}^{n f}\right)^{-1} & 0 \\
0 & 0
\end{array}\right)-\lambda \mathscr{M}^{\infty} \cdot\left(\begin{array}{cc}
\left(\tilde{\boldsymbol{R}}_{F U}^{n f}\right)^{-1} & 0 \\
0 & 0
\end{array}\right)\right]^{-1} } \\
& \cdot\left[\lambda \mathscr{M}^{\infty} \cdot\left(\begin{array}{c}
\left(\tilde{\boldsymbol{R}}_{F U}^{n f}\right)^{-1} \cdot\left(\boldsymbol{R}_{F E}^{n f}: \boldsymbol{E}^{\infty}+\boldsymbol{F}^{P}\right) \\
0
\end{array}\right)\right. \\
& \left.-\left(\begin{array}{c}
\left(\tilde{\boldsymbol{R}}_{F U}^{n f}\right)^{-1} \cdot\left(\boldsymbol{R}_{F E}^{n f}: \boldsymbol{E}^{\infty}+\boldsymbol{F}^{P}\right) \\
-\boldsymbol{E}^{\infty}
\end{array}\right)\right]
\end{aligned}
$$

and

$$
\boldsymbol{U}-\boldsymbol{u}^{\infty}=\left(\tilde{\boldsymbol{R}}_{F U}^{n f}\right)^{-1} \cdot\left(\tilde{\boldsymbol{F}}^{H, f f}+\boldsymbol{R}_{F E}^{n f}: \boldsymbol{E}^{\infty}+\boldsymbol{F}^{P}\right),
$$

where $\lambda$ is an arbitrary coefficient, $\tilde{\boldsymbol{R}}_{F U}^{n f}=\boldsymbol{R}_{F U}^{n f}+\lambda \boldsymbol{I}, \boldsymbol{I}$ is the idem tensor and $\tilde{\boldsymbol{F}}^{H, f f}=\boldsymbol{F}^{H, f f}+\lambda\left(\boldsymbol{U}-\boldsymbol{u}^{\infty}\right)$. Since $\boldsymbol{R}_{F U}^{n f}$ by itself is not always invertible, an additional diagonal tensor is added to make it positive definite. A value of $6 \pi \eta a$ for $\lambda$ will always suffice to make $\tilde{\boldsymbol{R}}_{F U}^{n f}$ invertible; however, larger values of $\lambda$ will yield a betterconditioned tensor at volume fractions approaching maximum packing.

\subsubsection{Accelerated Stokesian dynamics}

The accelerated Stokesian dynamics technique is an application of the so-called particle-mesh Ewald algorithm used regularly in computational physics to accelerate lattice sums (see e.g. Darden, York \& Pedersen 1993). Previous subsections have developed a solution for Stokes flow in a channel subject to a periodic though otherwise arbitrary body force, split that solution into wave-space and real-space contributions, which are both rapidly converging, and then attributed the body force to the force on the fluid due to colloidal particles in suspension. The aim in this 


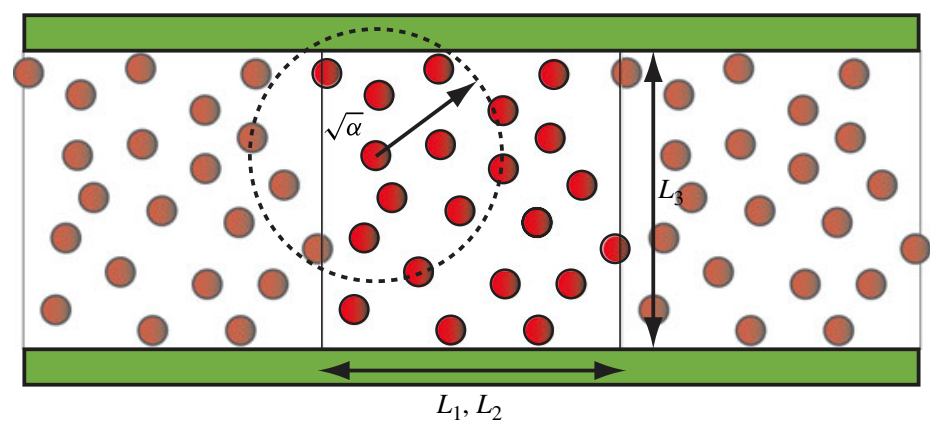

FIGURE 4. (Colour online) The periodic simulation cell has walls that are infinite in extent bounding the top and bottom of the channel. The interaction regime dominated by the local velocity field is indicated for one particle and characterized by the distance $\sqrt{\alpha}$.

subsection is to discuss one method of building a particle-mesh Ewald algorithm and emulating the accelerated Stokesian dynamics technique. This requires the calculation of three quantities: $(a)$ the real-space and $(b)$ the wave-space contributions to the disturbance velocity felt by particle $n$, which are characterized by (2.13) with substitution of the global force density and (2.39), respectively, and (c) the lubrication contributions to the resistance tensors denoted $\boldsymbol{R}_{F U}^{n f}, \boldsymbol{R}_{F E}^{n f}$ and $\boldsymbol{R}_{S E}^{n f}$, which reflect the near-field coupling of force and velocity, force and strain, and stresslet and strain, respectively. The first two quantities allow for the calculation of $\mathscr{M}^{\infty}$ via the Faxén formulae in (2.5), (2.6) and (2.7), while the remaining lubrication pieces complete the prerequisites for the Stokesian dynamics algorithm. As the simulation cell is twodimensional, the minimum image convention is used in determining the inter-particle separations for evaluation of lubrication interactions (i.e. the separation between a pair of particles is evaluated as the shortest distance in the set measured between one particle in the pair to the other and its periodic images).

Consider first the real-space contribution to the grand mobility tensor. Particle $n$ feels a disturbance flow from other particles in the fluid that decays exponentially fast with respect to $\left|\boldsymbol{x}-\boldsymbol{x}^{(n)}\right|$. Remember that this arises from the local force density and the reflection of that flow off the lower and upper walls of the channel, independently (see Appendix C). The rate of decay is of course modulated by the splitting parameter. However, for a sufficiently small value of $\alpha$, this rapid decay suggests that the realspace part of the flow affecting particle $n$ originates only from disturbances that are roughly $\sqrt{\alpha}$ away. Any further away, and the flows due to those disturbances have decayed to minuscule magnitudes and have no practical effect on the motion of particle $n$. This means that the computation of the real-space contribution to the flow felt by particle $n$ depends on disturbances generated by particles within a nearby neighbourhood roughly $\sqrt{\alpha}$ in extent. This lends itself quite nicely to the so-called linked cell method (also known as the chaining mesh method), which allows for exactly that sort of procedure (see e.g. Allen \& Tildesley 1989). Rather than checking the distances between all pairs of particles and computing the disturbance flows (something that requires $O\left(N^{2}\right)$ computations), the linked cell technique allows for computation of the flow due only to particles close enough to particle $n$ subject to some explicit cutoff distance (i.e. $\sqrt{\alpha}$ ). This is accomplished by dividing the periodic cell into sub-cells that are approximately $\sqrt{\alpha} \times \sqrt{\alpha} \times \sqrt{\alpha}$ in dimension and then binning the particles in their corresponding sub-cells. Only particles residing in the 
same sub-cell, or in a neighbouring sub-cell generate flows strong enough to affect one another. Therefore, the calculation of the real-space contributions to the disturbance flow around each and every particle can be completed in $O(N)$ operations.

Recall that a multipole expansion of the disturbance velocity generated by the particle produces a series of terms linear in the particle force moments and proportional to derivatives of the channel Green's function (see (2.44)). Rather than take these derivatives explicitly, one can follow the usual scheme of distributing the force moments as a series of point forces of an appropriate magnitude located on a self-similar grid (Hockney \& Eastwood 1989). This step is essential, as the efficiency of the algorithm is derived from the application of fast Fourier transformation methods on a regular grid. Consider the velocity field generated by a point force of magnitude $\boldsymbol{F}$ located at point $\boldsymbol{y}$,

$$
\sum_{k} \mathrm{e}^{-2 \pi \mathrm{i} k_{\alpha}\left(x_{\alpha}-y_{\alpha}\right)} \boldsymbol{G}^{(k)}\left(x_{3}, y_{3}\right) \cdot \boldsymbol{F},
$$

which can be approximated as a set of forces pointing in the same direction as $\boldsymbol{F}$ but located on the grid points denoted $\boldsymbol{y}^{(\gamma)}$ and of magnitude $A^{(\gamma)}$,

$$
\sum_{\gamma} A^{(\gamma)} \sum_{k} \mathrm{e}^{-2 \pi \mathrm{i} k_{\alpha}\left(x_{\alpha}-y_{\alpha}^{(\gamma)}\right)} \boldsymbol{G}^{(k)}\left(x_{3}, y_{3}^{(\gamma)}\right) \cdot \frac{\boldsymbol{F}}{|\boldsymbol{F}|} .
$$

A Taylor expansion of the grid approximate in terms of $\boldsymbol{y}^{(\gamma)}$ and about $\boldsymbol{y}$ results in a hierarchy of equations governing the grid coefficients such that, in one dimension,

$$
\sum_{\gamma}^{n} A^{(\gamma)}\left(y^{(\gamma)}-y\right)^{m}=\delta_{m 0},
$$

for $m=0,1,2, \ldots, n-1$ and where $\delta_{m 0}$ is the Kronecker delta-function. The algebraic structure here is easily recognizable - this is the transpose Vandermonde matrix operating on a vector of all the $A^{(\gamma)}$. The apparent algebraic analogy to leastsquares polynomial approximation is striking. The number of grid nodes $n$ defines the coarseness of the approximation such that the error is of the order of the $n$th power of the grid spacing. As it happens, the solution to these equations can be written as the superposition of unity and $n-1$ finite-difference stencils. For instance, the values of $A^{(\gamma)}$ in a one-dimensional approximation satisfying the grid hierarchy for $n=3$ are simply

$$
\left(-\frac{\Delta^{\prime}}{2 \Delta}+\frac{\Delta^{\prime 2}}{2 \Delta^{2}}, 1-\frac{\Delta^{\prime 2}}{\Delta^{2}}, \frac{\Delta^{\prime}}{2 \Delta}+\frac{\Delta^{\prime 2}}{2 \Delta^{2}}\right),
$$

where $\Delta$ is the spacing between nodes and $\Delta^{\prime}$ is the distance between the actual point source and the nearest grid node. This same procedure can be extended to any value of $n$ and, without loss of generality, the grid coefficients will be a superposition of weighted difference stencils of ever-increasing order. Since all but the zeroth-order grid equation sum exactly to zero, one physical interpretation is that the grid approximate is generated in such a way that the structure of force moments up to order $n-1$ is preserved. As the algorithm in this paper contains force moments up to the octuple, coefficients satisfying the grid equations to degree $n=4$ are necessary to maintain consistency. Incorporating higher-order force moments into this formulation is straightforward as well. The grid force density is written as being linearly proportional to the force multipole, and the same hierarchy of equations is 
constructed, though the single, non-zero summation corresponds to the force moment in question. An extension to three dimensions is not trivial, since any symmetric stencil will introduce a rank-deficient problem. This, however, may be solved in the least-squares sense such that a similar and unique set of finite-difference-like weightings emerge. This approach is generalizable to any Green's function and allows for arbitrary control of the accuracy of the mesh distribution.

Given a set of point forces on a grid, the calculation of the global contribution to the resulting disturbance velocity is uncomplicated. The process is begun by the fast Fourier transformation of each discretized plane of the force mesh parallel to the channel walls. For each transformed plane, the Fourier components of the disturbance velocity at that level due to all the point forces on the grid are calculated. Then the global disturbance velocity at each plane level is computed via an inverse fast Fourier transformation. Finally, the disturbance velocity located at any point in the simulation cell is approximated by a set of local Lagrange polynomials drawn from the grid-distributed global velocity field. Similarly, the derivatives of the disturbance velocity can be computed directly from the Fourier transformation of the gridded force or from derivatives of the interpolating polynomials. Note that the 'fineness' of the discretization in the $\boldsymbol{e}_{3}$ direction (perpendicular to the channel walls) is independent of the number of particles in the simulation cell for a fixed channel width and volume fraction. Therefore, the computation of the disturbance velocity and its derivatives is dominated by the fast Fourier transformations, which require $O(N \log N)$ calculations. Throughout the entire algorithm, this step alone has super-linear computational scaling and dominates the computation for simulations of a large number of particles.

It is undesirable to repeat the calculation of the wave-space contribution to the flow felt by every particle in the simulation cell owing to the disturbance from every other particle individually. This would require solving the problem for the disturbance velocity $N$ times and is computationally prohibitive. Instead, one computes the wave-space contribution to the flow generated by all the particles and then removes individually the 'self'-contribution due to the particle that 'feels' the flow. This quantity may by computed in advance as

$$
\boldsymbol{u}_{S}\left(\boldsymbol{x}_{n}\right)=\int_{V} \mathrm{e}^{-2 \pi \mathrm{i} k_{\alpha} x_{\alpha}^{(n)}} \boldsymbol{u}^{(k)}\left(x_{3}^{(n)}\right) \mathrm{d} \boldsymbol{k},
$$

where the Fourier coefficients of the force density generating $\boldsymbol{u}^{(k)}\left(x_{3}^{(n)}\right)$ are simply $\exp \left(2 \pi \mathrm{i} k_{\alpha} x^{(n)}\right) \boldsymbol{F}_{n}$. This quantity depends on one length scale, namely the channel width $\left(L_{3}\right)$, and two dimensionless parameters, $\Xi=x_{3}^{(n)} / L_{3}$ and $\beta=\alpha / L_{3}^{2}$. While $\Xi$ will always reside within the range of zero to unity, the rescaled splitting parameter is potentially boundless. However, in practice, $\sqrt{\alpha}$ of about three particle radii generates a sufficiently accurate approximation of the disturbance flows (see Sierou \& Brady 2001). The wave-space part of the disturbance flow generated and felt by a single particle, denoted again as $n$, is computed for values of $\beta$ ranging from 0.01 to 5, which is a diverse enough spread to study the motion of particles in rather wide channels and rather narrow channels, respectively. One simply applies the appropriate Faxén formulae to this particular disturbance velocity.

The channel geometry introduces an inherent anisotropy in the structure of the mobility tensors, and symmetry arguments suggest that the so-called 'self'-mobility tensors (denoted $\boldsymbol{M}_{U F}^{S}, \boldsymbol{M}_{U D}^{S}, \boldsymbol{M}_{\nabla F}^{S}$ and $\boldsymbol{M}_{\nabla D}^{S}$ for the coupling of velocity and force, velocity and doublet, gradient velocity and force, and gradient velocity and doublet) 
have a well-defined structure, for instance,

$$
\begin{aligned}
\boldsymbol{M}_{U F}^{S}= & -\frac{1}{6 \pi \eta a_{n}}\left\{\left[f_{1}^{(U F)}(\Xi, \beta)\left(\frac{a_{n}}{L_{3}}\right)-f_{3}^{(U F)}(\Xi, \beta)\left(\frac{a_{n}}{L_{3}}\right)^{3}\right.\right. \\
& \left.+f_{5}^{(U F)}(\Xi, \beta)\left(\frac{a_{n}}{L_{3}}\right)^{5}\right]\left(\boldsymbol{I}-\boldsymbol{e}_{3} \boldsymbol{e}_{3}\right) \\
& +\left[g_{1}^{(U F)}(\Xi, \beta)\left(\frac{a_{n}}{L_{3}}\right)-g_{3}^{(U F)}(\Xi, \beta)\left(\frac{a_{n}}{L_{3}}\right)^{3}\right. \\
& \left.\left.+g_{5}^{(U F)}(\Xi, \beta)\left(\frac{a_{n}}{L_{3}}\right)^{5}\right] \boldsymbol{e}_{3} \boldsymbol{e}_{3}\right\},
\end{aligned}
$$

while the structures of the others are described in Appendix D. For simplicity, the doublet to which the torque and the stresslet are the antisymmetric and symmetric contributions is introduced. The gradient velocity to which the rate of rotation and rate of strain are similarly related is used as well. This, of course, is the same structure that was observed in the reflection of the flow generated by a single particle in the parallelwall geometry by Swan \& Brady (2010). However, since the simulation of suspensions has required a more sophisticated analysis, the disturbance flow is modulated by the splitting parameter. The velocity-force couple is plotted in figure 5 as a function of $\Xi$ for a few values of $\beta$ in the range described.

In practice, however, the difference between the actual mobility for a single particle in the parallel-wall geometry $(\beta \rightarrow 0)$ and the wave-space contribution to that mobility for the specified value of the splitting parameter is all that is required. Perhaps the most efficient computational implementation of this is via tabulation of the quantities: $\boldsymbol{M}^{S}(\beta \rightarrow 0)-\boldsymbol{M}^{S}(\beta)$. Note that any explicit dependence on the channel width has been removed from the calculation and reduced the dimensionality of the tabulation. Additionally the table is bounded by fixed limits for the values of $\Xi$ and by practical but flexible limits on the values of $\beta$.

The lubrication contributions to the resistance tensors play a critical role in the dynamics and rheology of colloidal dispersions. As a pair of particles nearly touches or a single particle passes near a wall, the resistance to relative motion of that pair or motion of that particle is singular. This arises from the large pressure gradients required to squeeze fluid out of the narrow gap separating two impenetrable surfaces. For many particles near one another, these singularities are effectively pairwise additive since the fluid in a gap between two nearly touching surfaces is to a first approximation independent of the fluid in the other gaps. While the same does not hold for the regular contributions to the resistance, numerous studies have found that treating the lubrication contributions to the resistance as pairwise additive regardless produces quantitatively accurate results (see e.g. Brady et al. 1988; Bossis, Meunier \& Sherwood 1991; Phung, Brady \& Bossis 1996). In fact, this approach is the touchstone in the field. To that end, the pairwise (i.e. particle-particle and particle-wall) singular contributions are used to construct the resistance tensors $\boldsymbol{R}_{F U}^{n f}, \boldsymbol{R}_{F E}^{n f}$ and $\boldsymbol{R}_{S E}^{n f}$ (see e.g. O'Neill \& Stewartson 1967; Happel \& Brenner 1986; Kim \& Karrila 2005). These are well-known quantities that are readily available in the cited literature. 


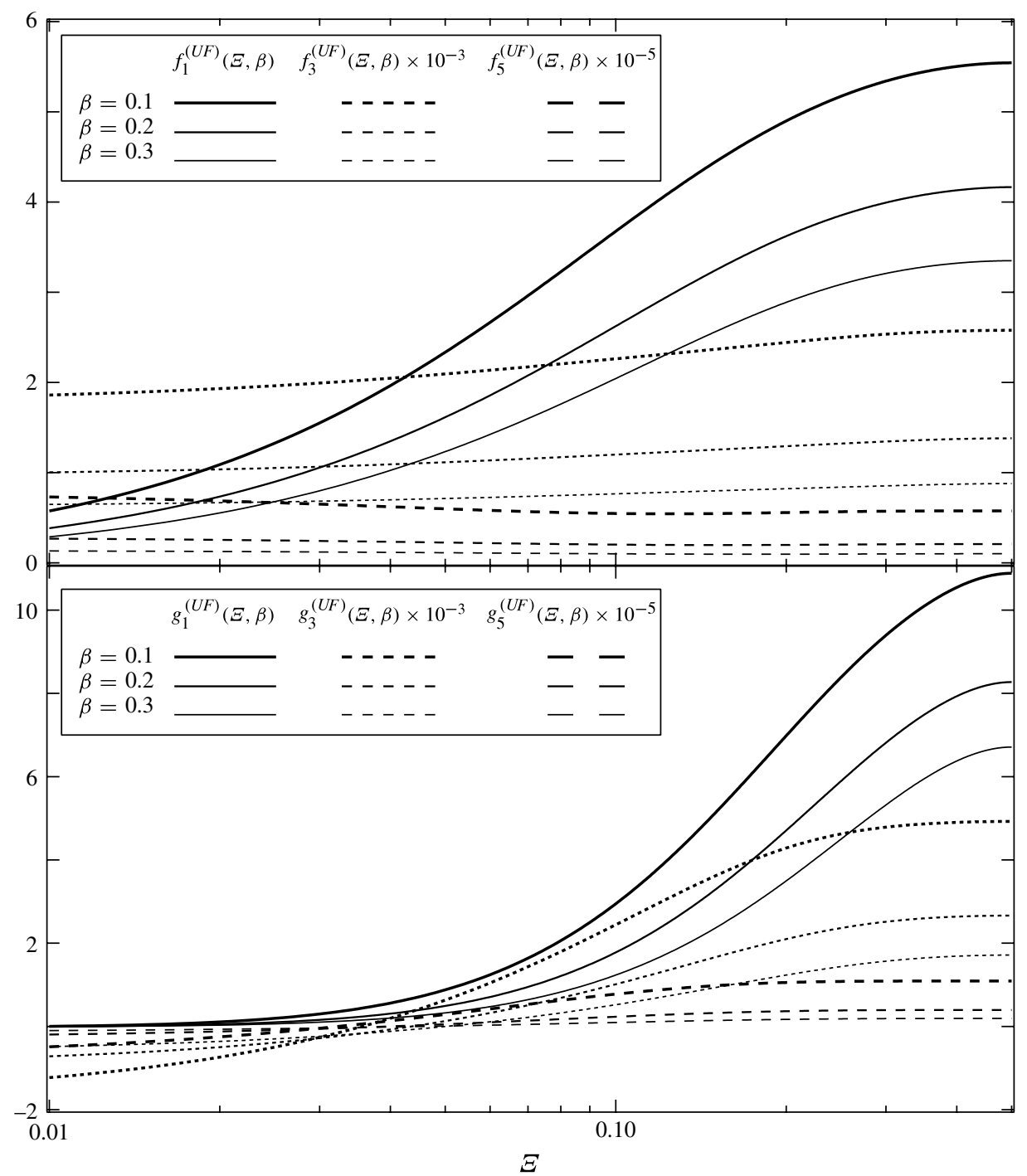

FIGURE 5. The so-called 'self'-contribution to the mobility due to the wave-space disturbance flow depends on both the dimensionless splitting parameter and the fractional distance across the channel. This can be computed and tabulated easily for all values of these two parameters, which are effectively independent of the channel width in much the same manner as in Swan \& Brady (2010).

\section{Applications}

There are relatively few measurements of the short-time properties of confined suspensions (either analytical, computational or experimental) beyond the dilute regime. However, knowing that, as the channel widens, the viscoelastic properties of a suspension cannot deviate from their equivalent in the well-studied, unbounded limit serves as a useful guide. Still, a direct comparison between this simulation method and one other concerning the cooperative motion of particles in a channel is possible. Save that, predictions are made of the high-frequency viscosity, short-time self-diffusivity 


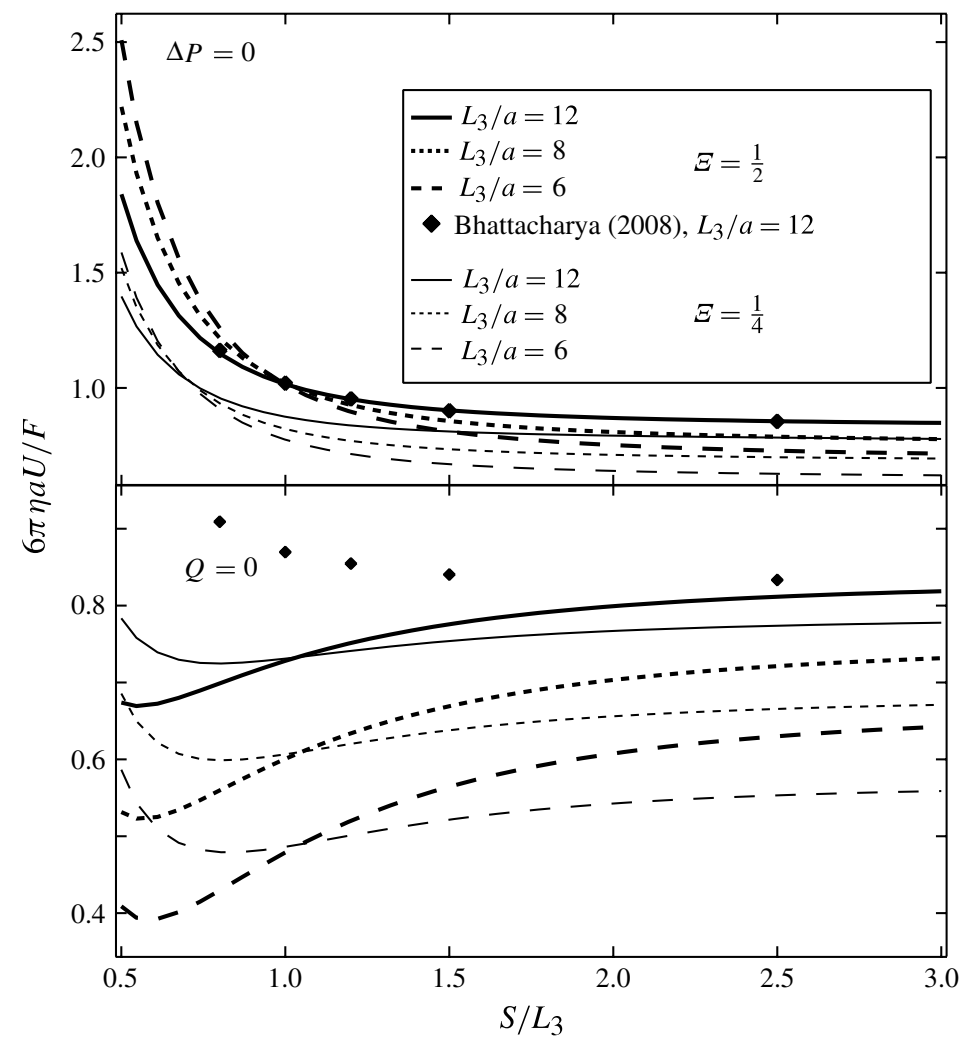

FIGURE 6. The sedimentation rate of spherical particles residing on a square lattice falling parallel to the channel walls. The rates for square lattices, with lattice dimension $S$, residing in the middle of the channel, $\Xi=1 / 2$, and a quarter of the way across the channel, $\Xi=1 / 4$, are qualitatively the same, though lattices nearer to the wall fall slower due to the increased drag.

and sedimentation rate, which must be compared with and analysed in the context of those for unbounded suspensions.

\subsection{Cooperative motion of regular lattices}

Bhattacharya (2008) used the method of moments approach originating with Cichocki et al. (2000) and Bhattacharya \& Blawzdziewicz (2002) to study the cooperative motion of particles arranged in an infinite square or rectangular lattice with lattice dimension $S$ residing on the centre plane of a parallel-wall channel. In that work, only the motion parallel to the channel walls was studied for channels with a width of 12 particle radii $\left(L_{3}=12 a\right)$. Here, both the cooperative motion parallel and perpendicular to the channel walls (i.e. the sedimentation rate of the lattice) is measured as a function of the channel width and the square lattice spacing.

In figure 6 , the parallel sedimentation rate relative to the particle weight $(6 \pi \eta a U / F)$ is plotted for two cases: one where the applied pressure difference $(\Delta P)$ down the channel is zero and one where the mean flow of material down the channel $(Q)$ is zero.

With no applied pressure gradient, the present method reproduces the predictions of Bhattacharya (2008) exactly. The same cannot be said of the predictions of collective 
motion in the 'no-flow' limit. While the present prediction reflects the behaviour typical of sedimentation of fully three-dimensional lattices (for which the condition $Q=0$ is implicit), those of Bhattacharya (2008) trend in the opposite direction. They find that, as the lattice becomes more concentrated, it falls faster. It should be the case that the back-flow hinders concentrated suspensions more than dilute ones, as the pressure gradient generating that parabolic flow balances the weight of the particles exactly (i.e. the sum of the forces causing the particles to move collectively). While the exact expression for the back-flow pressure in the channel is given by (2.26), the behaviour in both the dilute $(S \rightarrow \infty)$ and the concentrated $(S \rightarrow 2 a)$ regimes can be estimated with simple analysis. In the dilute case, the sedimenting particles represent a body force in the channel with number density $1 / S^{2} L_{3}$. The volume of fluid dragged by this body force can be estimated from the zero-wavevector solution to the Stokes equations (2.24). The back-flow must cancel the volume flux due to the dragged fluid and the sedimenting particles, which fall at the same speed as a single particle in a channel with an order $1 / S^{2}$ hindrance due to the other particles. In this case, the particles are slowed by a factor

$$
U(\Delta P=0)-U(Q=0)=\frac{F}{6 \pi \eta a}\left(\frac{a}{S}\right)^{2}\left[18 \pi \Xi^{2}(1-\Xi)^{2}\left(\frac{L_{3}}{a}\right)\right]
$$

relative to their fall speed were there no pressure gradient applied to the channel. In the case that the channel is large, this quantity diverges just as in isolated Stokes flow $\left(a / L_{3} \rightarrow 0\right)$. We see from (3.1) that dilute lattices fall slower as the lattice dimension shrinks under the zero volume flux condition. Similarly, a concentrated lattice must have the same physical behaviour as a wall of width $2 a$ falling down a channel in which the back-flow pressure gradients on either side of the wall must be equal. The ratio of the fall speed with no applied pressure gradient to that with zero volume flux is

$$
\frac{U(\Delta P=0)}{U(Q=0)}=\frac{\left(L_{3} / a-2\right)\left(L_{3} / a+2\right)}{1+\left(L_{3} / a\right)[3 \Xi(1-\Xi)-1]}-\frac{4}{L_{3} / a-2} .
$$

For $\Xi=1 / 2$, this ratio is $(7,6,5.2)$ respectively with $L_{3} / a=(6,8,12)$. From simulations with $S=2.0001 a$, we find that this ratio is $(6.4,5.9,4.6)$ for the same channel widths. The discrepancy with Bhattacharya (2008) must be explained by the particular assumptions and approximations they employed.

Nearer the wall and for no mean flow $(Q=0)$, an interesting trend emerges. While the sedimentation rate drops as the number density increases, there is a point beyond which the bare fall speed of the particles supersedes the back-flow, which itself falls off quadratically from the channel centre. As a result, a lattice near the wall can fall faster than the same lattice mid-channel. It is easier for a concentrated lattice to slip through the fluid, as the streamlines curve less around the lattice particles. As a result, the back-flow hinders the particles less efficiently. It is the interplay of this effect and the hydrodynamic interactions between the lattice and the nearby wall that gives rise to this behaviour.

The collective motion of a square lattice perpendicular to the channel walls is an entirely different matter and is plotted in figure 7 . There is always the condition of 'no flow' in the direction normal to the channel walls, and there is always a pressure gradient implicitly exerted to balance the weight of the sedimenting particles. However, and unlike unbounded sedimentation, the lattice moves relative to the nearby channel walls so that fluid is always forced to pass between the particles. Consequently, more concentrated lattices sediment at a significantly slower rate than an isolated particle. 


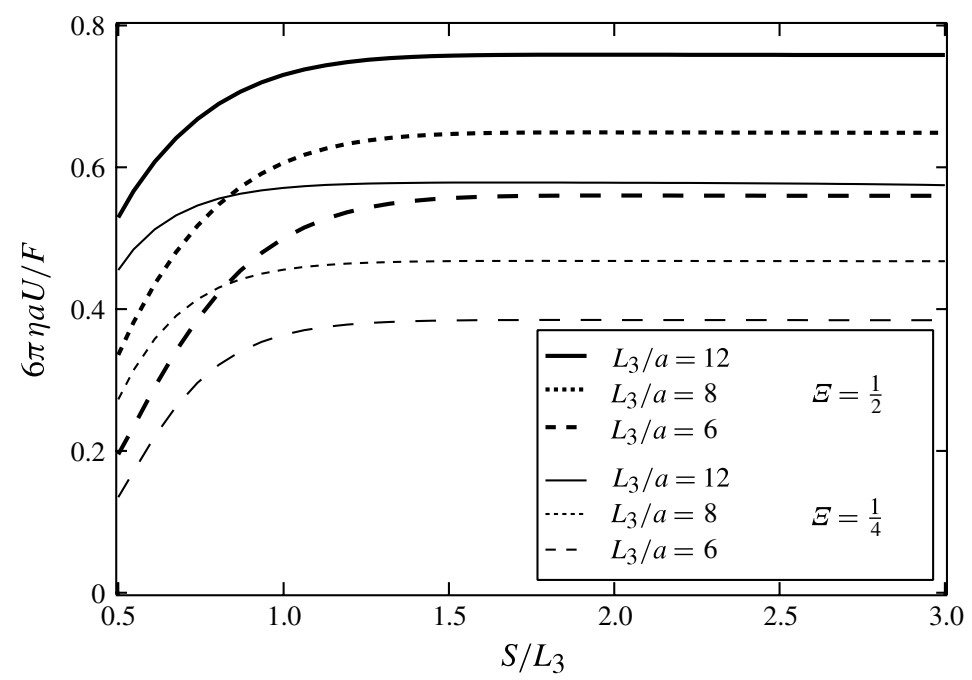

FIGURE 7. The sedimentation rate of spherical particles residing on a square lattice falling perpendicular to the channel walls at positions one-half and one-quarter $(\Xi=1 / 2$ and $\Xi=1 / 4$, respectively) across the channel. Fluid must squeeze through the lattice gaps for particles to come nearer the walls in order to satisfy continuity throughout the domain; therefore, more concentrated lattices fall more slowly.

Again, this is in accord with what is anticipated in situations where a zero mean flux constraint is imposed.

For motion either parallel or perpendicular to the channel walls and regardless of restrictions on the flow, the enhancement of or hindrance to sedimentation decays rapidly with respect to the ratio of lattice spacing to channel width $\left(S / L_{3}\right)$. In fact, above a ratio of approximately 2 , the lattice sedimentation rate is within just a few per cent of the single-particle sedimentation rate in all the cases simulated. This is an important observation, as it is well known that the measurement of suspension diffusivity is skewed in periodic systems by precisely these cooperative effects. Given the more rapid decay of the disturbance generated by a point force in a channel and these observations, one can conclude that measurements of diffusivity in periodic channels with an aspect ratio larger than 2 are within only a few per cent of what could be expected in the limit that the aspect ratio approaches infinity. This belies the strong $\left(S^{-1}\right)$ dependence of the sedimentation rate for a three-dimensional cubic lattice on the lattice dimension and is a consequence of the hydrodynamic screening induced by the channel walls. The preceding examples all adhere to the heuristic that the lateral dimensions of the simulation cell, $L_{1}$ and $L_{2}$, are equal and that $L_{1} / L_{3}>2$. Consequently, the simulations are in the regime where the effects of periodicity are weak. In the limit that $S / a \rightarrow \infty$, the lattice sedimentation rate converges to the fall speed of a single particle regardless of the condition at the channel ends.

In figure 8 , the convergence of the algorithm towards the known result due to Bhattacharya (2008) for no applied pressure gradient is demonstrated by varying both the splitting parameter and the number of nodes used to discretize the wave-space solution. In particular, for a channel 12 particle radii across, 32 nodes are used in the direction normal to the wall, while the number of nodes parallel to the wall is varied from $2^{4}$ to $2^{7}$. As the lattice dimension varies, the density of nodes parallel to the wall, denoted $M / a$, changes as well. Similarly, four distinct values of the 


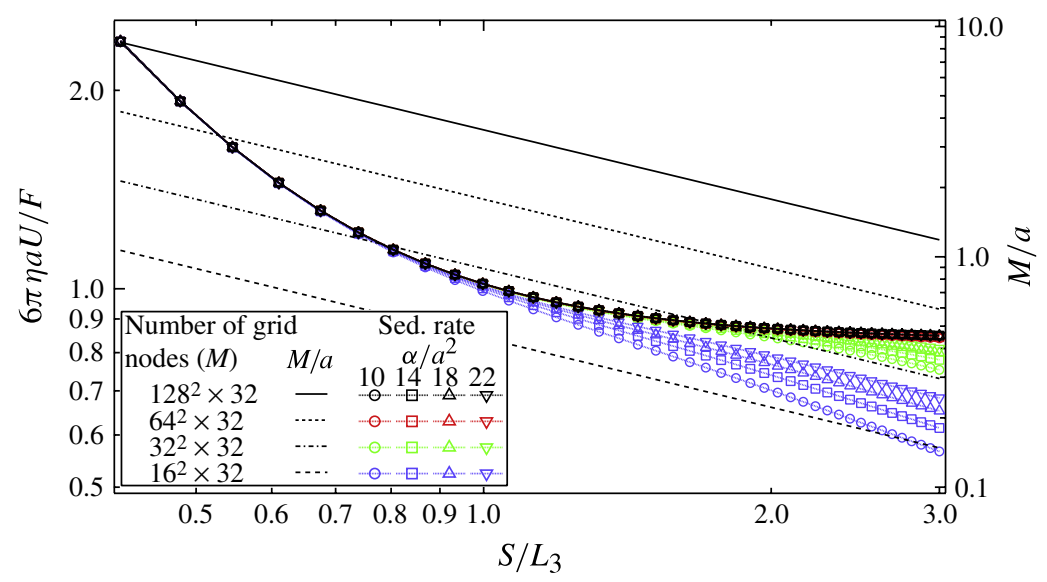

FIGURE 8. (Colour online) As in figure 6, the sedimentation rate along the channel with no applied pressure gradient is plotted. It was previously demonstrated that this calculation reproduces the known result due to Bhattacharya (2008). In this case, however, the empiricism employed throughout the simulations in this section (i.e. $\alpha=L_{3}^{2} / 8$ and a minimum of wave-space discretization density of two nodes per particle radii) is justified by demonstrating the algorithm's stability and convergence as the splitting parameter and wavespace discretization are varied. Note that there is virtually no difference in the sedimentation rate for discretizations of $64^{2} \times 32$ and $128^{2} \times 32$. Hence they may be difficult to distinguish on the plot.

splitting parameter are employed. The value chosen for all the results discussed in this section is $\alpha=L_{3}^{2} / 8$, which in this particular case is 18 . When the density of wave-space nodes is greater than one per particle radius, the predicted sedimentation rate is virtually identical for all discretization choices and splitting parameters. For less dense discretizations, the predicted sedimentation rate is smaller than the converged values, and smaller values of the splitting parameter yield less accurate results. This is to be expected, as a smaller splitting parameter necessitates finer discretization in wave space. Additionally, because the real-space interactions are calculated only for particle pairs within some cutoff distance larger than $\sqrt{\alpha}$, there is an insufficient number of terms in the real-space summation for larger values of $\alpha$. Rather than using an explicit cutoff for the real-space interaction, all the interactions can be calculated and a more accurate result generated for a given value of the splitting parameter. However, the efficiency of the algorithm suffers, as such a calculation requires $O\left(N^{2}\right)$ operations and is unsuitable for simulations of suspensions with more than 100 particles. A trade-off is necessary, but the validating figure demonstrates that there is an easily accessed region of parameter space (i.e. number of Fourier nodes and splitting parameter) for which the algorithm is within fractions of a per cent of the established result. Throughout these results, an empirical standard for the wave-space discretization, a minimum of two nodes per particle radius, is practised. This same standard was employed with success in the calculations of Sierou \& Brady (2001) for systems of unbounded colloids, and according to the figure is more than sufficient for accurate calculation of lattice sedimentation and by extension other suspension properties.

\subsection{Equilibrium suspension structure}

For the following suspension properties, equilibrium configurations of particles between the channel walls were generated using the Monte Carlo method for 


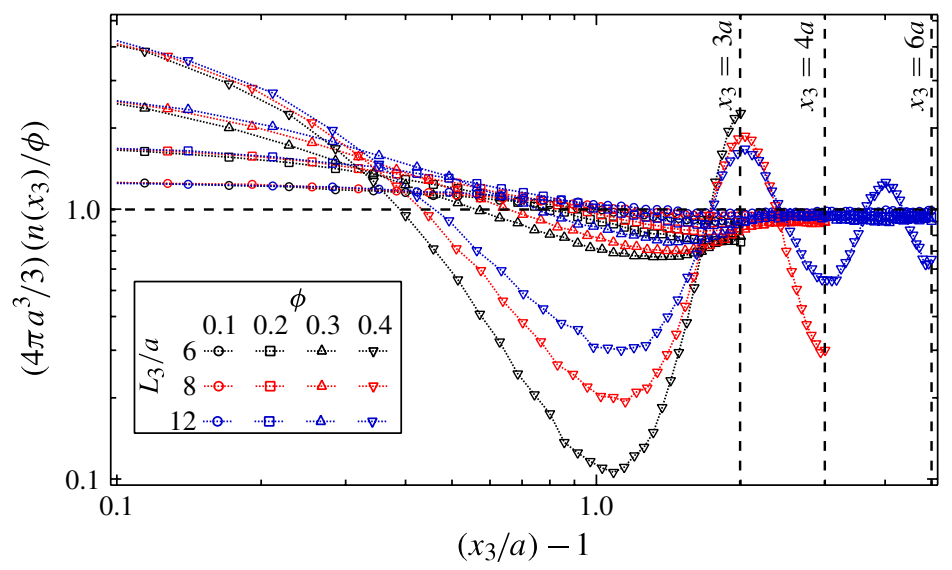

FIGURE 9. (Colour online) The equilibrium density profile across the channel $n\left(x_{3}\right)$ for several bulk channel volume fractions and channel widths. Notice the systematic deviations in the density profiles at high volume fractions. These structural variations can have a strong influence on the short-time rheology of concentrated suspensions.

volume fractions less than $30 \%$ (Frenkel \& Smit 2001) and via molecular dynamics simulation for volume fractions greater than $30 \%$ (Donev, Stillinger \& Torquato 2005). Figure 9 depicts the distribution of number density, $n\left(x_{3}\right)$, at equilibrium across channels with a variety of widths for many 'bulk' or averaged volume fractions, $\phi=\frac{4}{3} \pi a^{3} \int_{0}^{L_{3}} n\left(x_{3}\right) \mathrm{d} x_{3} / L_{3}$. This measure, like all the suspension properties computed in this paper, is the result of performing an ensemble average over 500 realizations of particle configurations drawn from the equilibrium, hard-sphere distribution. These static properties depend intimately on the structure of the suspension between the channel walls. However, the effects of packing and confinement on the equilibrium structure of the suspension are beyond the purview of this paper. The presented results are limited to volume fractions less than $40 \%$, because the walls induce ordering in the suspension that may trigger crystallization. One can understand this by considering that, while the number density of particles in the channel is $3 \phi /\left(4 \pi a^{3}\right)$, the centres of the particles cannot access areas nearer the wall than one particle radius. Violating this would lead to overlap with the hard walls. As such, the centre accessible number density is $\left[3 \phi /\left(4 \pi a^{3}\right)\right] L_{3} /\left(L_{3}-2 a\right)$, which, for channels not much wider than a particle diameter, can be significantly larger than the bulk value, hence, the induction of crystallization at lower bulk volume fraction.

\subsection{High-frequency dynamic viscosity}

The high-frequency dynamic viscosity is the result of an experiment where one of the channel walls is oscillated rapidly and with small amplitude. The time-averaged ratio of the speed of the wall to the force required to push it is proportional to the high-frequency dynamic viscosity, denoted $\eta_{\infty}^{\prime}$. Since this is done in the limit that the oscillatory time scale is shorter than all other time scales, the motion of the particles is inconsequential and what is actually probed is simply the mean particle stresslet for an equilibrium configuration of particles, viz.

$$
\frac{\eta_{\infty}^{\prime}}{\eta_{0}}=1-\frac{1}{2 \eta \boldsymbol{E}^{\infty}: \boldsymbol{E}^{\infty}} \int_{0}^{L_{3}} n\left(x_{3}\right)\langle\boldsymbol{S}\rangle_{x_{3}}: \boldsymbol{E}^{\infty} \mathrm{d} x_{3},
$$




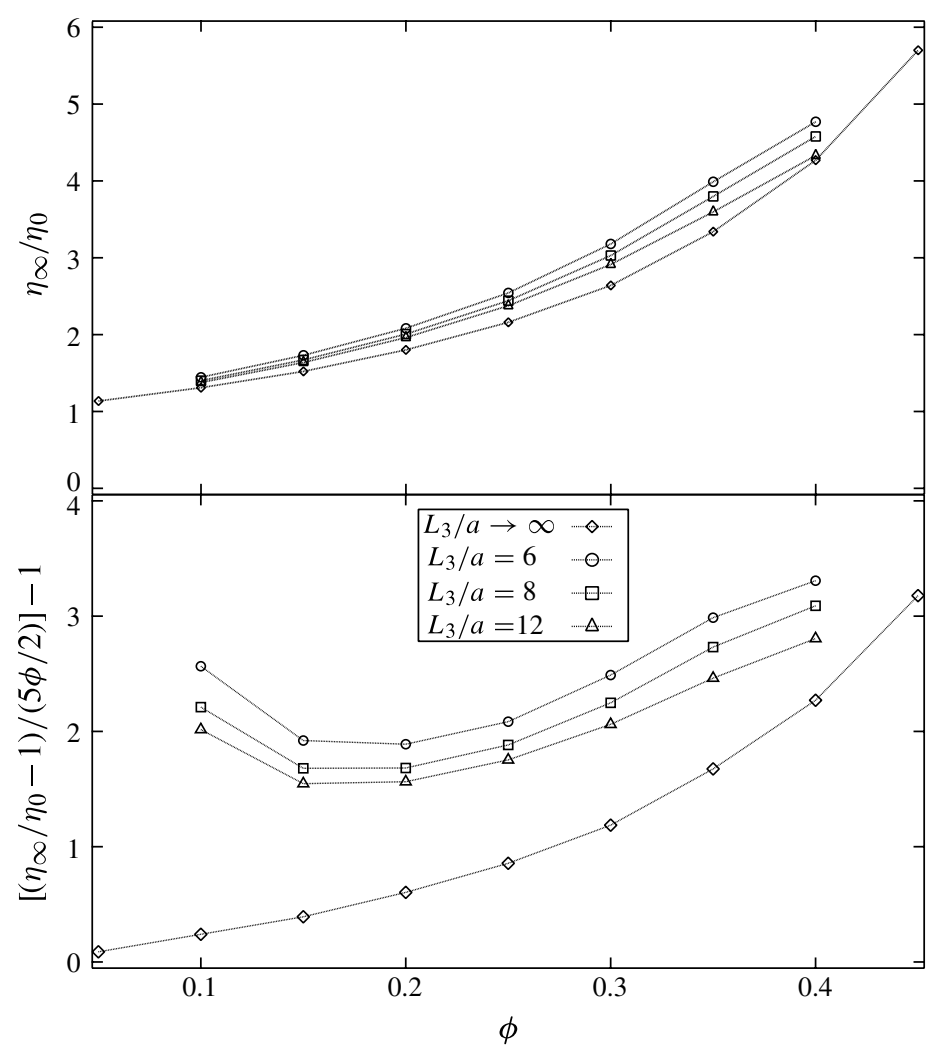

FIGURE 10. The high-frequency dynamic viscosity and viscosity increment less the Einstein contribution $(5 \phi / 2)$ plotted as a function of volume fraction and channel width. Note the nonmonotonic trend in the viscosity increment. This is due to a combination of hydrodynamic screening and the additional resistance due to the channel walls.

where the average $\langle\boldsymbol{S}\rangle_{x_{3}}$ is the mean particle stresslet conditioned over a particular position $\left(x_{3}\right)$ in the suspension at equilibrium. In particular, one is interested in the response to a shear flow generated by differential translation of the channel walls. In that case, the only non-zero components of $\boldsymbol{E}^{\infty}$ are the $\boldsymbol{e}_{1} \boldsymbol{e}_{3}$ and $\boldsymbol{e}_{3} \boldsymbol{e}_{1}$ dyads. The highfrequency viscosity is plotted as a function of suspension volume fraction and channel width in figure 10. Additionally, the increment to the Einstein viscosity $[\eta(1+5 / 2 \phi)]$ is measured and plotted. This is an $O(\phi)$ quantity, since there is additional dissipation associated with the motion of a single particle in the channel that is not present in an unbounded, dilute suspension.

Notice that, at low and high particle concentrations, and regardless of channel width, the high-frequency viscosity is near that of an unbounded suspension. At moderate volume fractions, there is a systematic deviation, which grows as the channel width decreases. While at high volume fractions it is the particle-particle lubrication that dominates the dissipation, at low and moderate volume fractions, the lubrication interactions with the walls substantially increment the suspension viscosity. This is borne out by the trend in the viscosity with respect to channel width: the narrower the channel, the larger the fraction of particles near the wall and therefore the larger the viscosity increment. Interestingly, however, the measured deviation from the bulk viscosity is not more than $15 \%$ at low volume fractions and decreases to only a few 


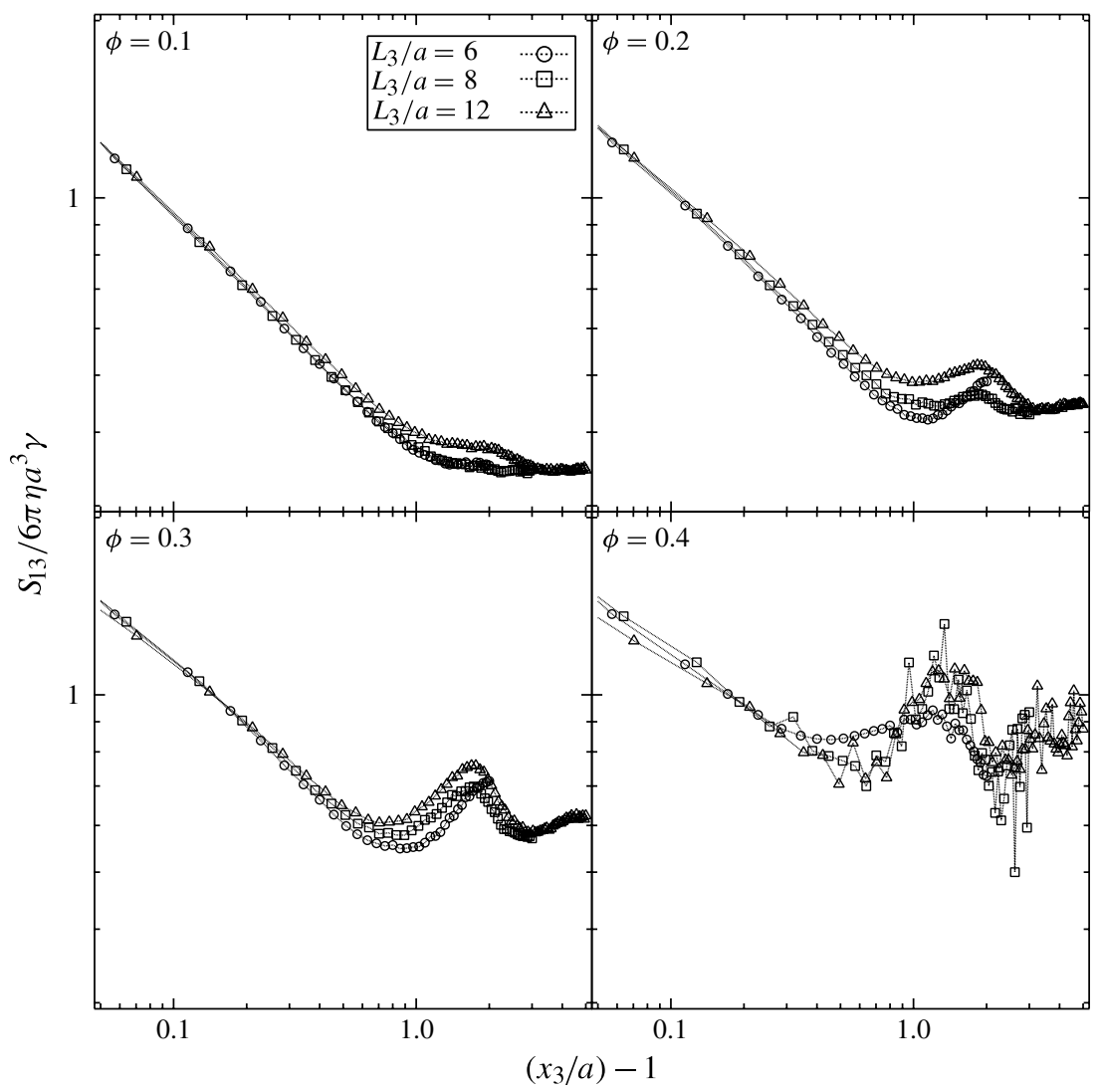

FIGURE 11. The distribution of particle stresslet across channels of varying width for different volume fractions. Notice that the near-wall contribution is effectively invariant while the mid-channel contribution grows with increasing volume fraction. The figure axes are logarithmic so that the dimensionless stresslet is plotted between 0.3 and 2 .

per cent at higher volume fractions. These trends are directly observable in a plot of the mean stresslet distribution as a function of position across the channel, $\boldsymbol{e}_{1} \boldsymbol{e}_{3}:\langle\boldsymbol{S}\rangle_{x_{3}}$ (see figure 11).

While, near the wall, the mean particle stresslet is effectively invariant with respect to both channel width and volume fraction (a consequence of lubrication), in the midchannel region, the mean stresslet grows with volume fraction. As the suspension becomes more concentrated, the inter-particle spacing decreases, and lubrication interactions between the particles contribute significantly to the mean stresslet. It is important to recognize that the particle contribution to the suspension stress is the integral of the product of this stress distribution and the number density distribution. Therefore, even though the near-wall stresslet is invariant, it is more heavily weighted in concentrated suspensions since there are more particles near the wall. So, in contrast to unbounded suspensions, the single-particle structure plays an important role in determining the suspension stress. Notice, too, that, for concentrated systems, the suspension stress begins to approach a nearly constant value, as there is little difference between the lubrication interactions between a particle pair and between a particle and a plane wall. Thus, in channels larger than 12 particle radii, deviations 
from the bulk behaviour are likely to be difficult to discern experimentally. The same, however, is not true of the particle dynamics, as discussed next.

\subsection{Short-time self-diffusivity}

The short-time self-diffusivity is defined in terms of a particle's mean-squared displacement as

$$
D_{0}^{S}=\lim _{t \rightarrow 0} \frac{1}{2} \frac{\mathrm{d}}{\mathrm{d} t}\langle\boldsymbol{x}(t) \boldsymbol{x}(t)\rangle,
$$

where the angle brackets indicate an ensemble average typically over an equilibrium distribution for the particle positions. Here, the limit $t \rightarrow 0$ signifies a time scale short with respect to $6 \pi \eta a^{3} / k T$, the Brownian relaxation time of the particle, and long relative to $m / 6 \pi \eta a$, the inertial relaxation time of particles with mass $m$. From the fluctuation-dissipation theorem, this is equivalent to measuring the velocity of a single particle in a suspension propelled by a force proportional to the thermal energy $k T$. It also constitutes the diagonal elements of $k T\left\langle\boldsymbol{R}_{F U}^{-1}\right\rangle$ and therefore need not be computed explicitly via dynamic simulation. Instead, we measure the short-time self-diffusivity using the stochastic process first introduced by Sierou \& Brady (2001). Each particle is propelled by an independent and identically distributed random force such that there is no correlation of the forces among different particles and the covariance matrix for each particle force is diagonal. An ensemble average of the product of the resulting particle velocities and their forces can be shown to measure the diagonal components of $\boldsymbol{R}_{F U}^{-1}$. Averaged over many equilibrium particle configurations, this is simply the short-time self-diffusivity. As the distance of each particle from the wall is known explicitly, the dependence of the short-time self-diffusivity on position in the channel is accessible. Similarly, since the components of the individual random forces are uncorrelated, the diffusion parallel and perpendicular to the walls is also apparent. In fact, it is the inhomogeneous and anisotropic short-time self-diffusivity, denoted $\boldsymbol{D}_{0}^{S}\left(\phi, x_{3} / a ; L_{3} / a\right)$, that distinguishes the dynamics of a confined system from that of a bulk material. This quantity is plotted for several bulk volume fractions in figure 12 . For all cases, $L_{1} / L_{3}>2$, so that we do not need to worry about taking the limit $N \rightarrow \infty$. Rather, it was observed that the back-flow generated by periodicity is weak in the regime where the simulation cell has an aspect ratio larger than 2 (see §3.1) - a consequence of hydrodynamic screening by the confining walls.

We anticipate that, for concentrated systems, the influence of the walls is minimal. As such, over much of the channel the short-time self-diffusivity should deviate little from its bulk value, denoted $D_{0}^{S}(\phi)$. While, for dilute systems, the channel screens the long-range hydrodynamic interactions, so the hindrance to the particle dynamics is due primarily to the channel walls themselves as well as lubrication interactions with a few nearby particles. In this case, the short-time self-diffusivity would be a product of its bulk value and the mobility of a single particle in the channel, viz.

$$
\boldsymbol{D}_{0}^{S}\left(\phi, \frac{x_{3}}{a} ; \frac{L_{3}}{a}\right) \approx 6 \pi \eta a \boldsymbol{M}_{U F}^{S}\left(\frac{x_{3}}{a} ; \frac{L_{3}}{a}\right) D_{0}^{S}(\phi) .
$$

In essence, the dynamics would be those of a single particle in a channel with the same width but in a solvent of effective viscosity $k T / 6 \pi a D_{0}^{S}(\phi)$. While these two models, $D_{0}^{S}(\phi)$ at high volume fractions and (3.5) at low volume fractions, seem intuitive, applicability with respect to the width of the channel is not obvious. For instance, in the widest channels, the short-time self-diffusivity should converge to the bulk value and the two models become equivalent. However, when the 




FIGURE 12. (Colour online) The short-time self-diffusivity of particles in suspensions of volume fraction $\phi=0.1,0.2,0.3$ and 0.4 as a function of the distance across the channel and channel width. Note that, near the wall, the channel width is a relatively unimportant factor in setting the scale of the self-diffusivity for all volume fractions, while far from the channel walls, there is an intimate relationship between the suspension structure and the hydrodynamic interactions among the particles and with the channel walls. The figure axes are logarithmic so that the dimensionless dimensionless diffusivity is plotted between 0.04 and 0.7 .

channel becomes more narrow, not only does the structure of the dispersion change dramatically, but the influences of wall and inter-particle hydrodynamic interactions become indistinguishable. The consequence is that neither model can predict suitably the dynamics of the suspension. The effectiveness and breakdown of these models is illustrated by figures $13-15$.

Three channel widths $\left(L_{3} / a=6,8,12\right)$ and three volume fractions $(\phi=$ $0.1,0.25,0.4)$ are considered. For the widest channel and the lowest volume fraction (figure 13, $\phi=0.1$ ), the single-particle approximation (3.5) is virtually an exact match for the simulation data. Similarly, for the widest channel and the highest volume fraction $(\phi=0.4)$, the bulk approximation for the self-diffusivity is an excellent prediction, as the diffusivity is isotropic over much of the channel. For the moderately concentrated suspension $(\phi=0.25)$ in the widest channel, however, the self-diffusivity is nearly isotropic but far smaller than the bulk prediction. Similarly, it is larger than either the parallel or perpendicular single-particle approximations. As the channel 


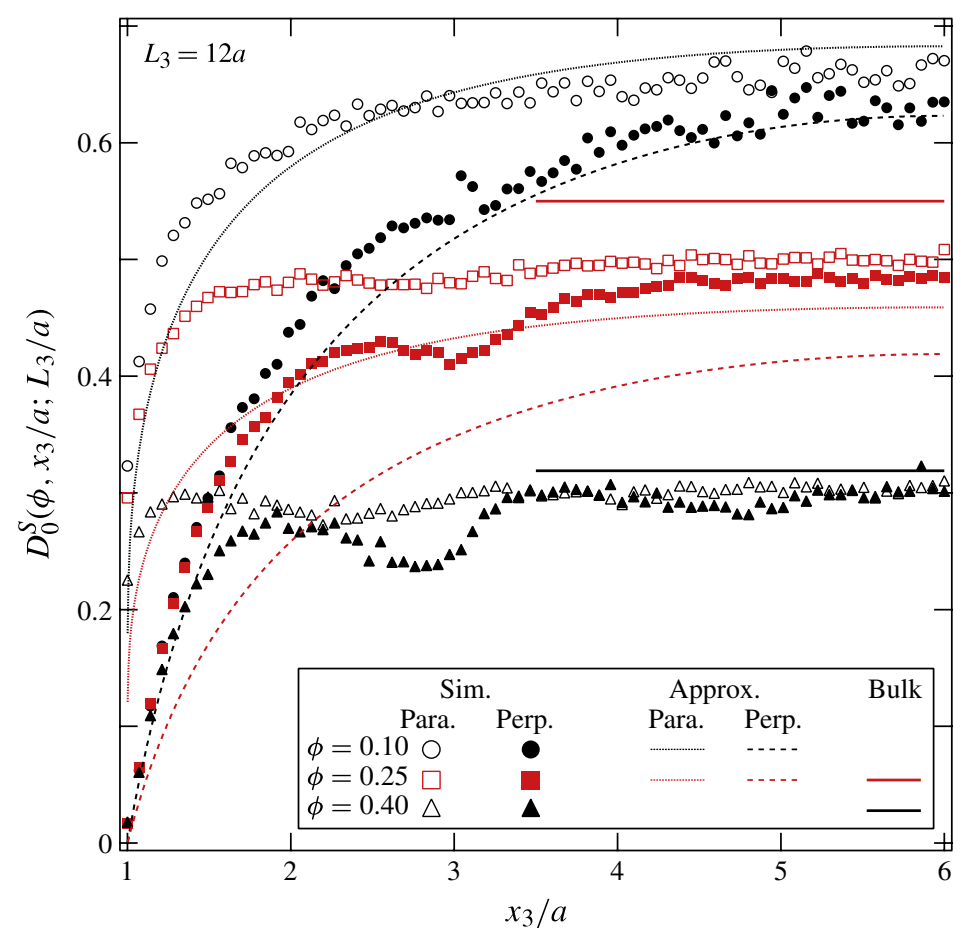

FIgURE 13. (Colour online) The short-time self-diffusivity is measured via simulation and compared to an approximation for the diffusivity of a single particle in the channel for an effective viscosity $k T / 6 \pi a D_{0}^{S}(\phi)$ as well as the bulk short-time self-diffusivity. This is a computation for $L_{3}=12 a$.

shrinks, these same trends persist (figure 14), though it appears that the bulk prediction for the highest volume fraction remains a better model. For the smallest channel studied (figure $15, L_{3} / a=6$ ), neither approximation is suitable for the most and least concentrated suspensions, and, throughout, the dynamics of moderately concentrated suspensions are not well approximated.

There is more to be drawn from these figures, however. The bulk diffusivity is always an overestimate of the diffusivity mid-channel (for $\phi=0.1$, the bulk diffusivity is $\approx 0.82$ and is off the scale of all the graphs). This is because the bulk limit does not account for the energy dissipated due to the no-slip condition on the channel walls. This additional hindrance is always present and always larger than that resulting from replacing the solid wall with a porous structure such as a collection of other colloidal particles. Similarly, rescaling the solvent viscosity on the bulk diffusivity and measuring the diffusivity of a single particle in the equivalent channel filled with the fictitious fluid always underestimates the self-diffusivity. This is because the inter-particle hydrodynamic interactions are actually weaker than the bulk limit predicts, as they are screened by the walls (i.e. the mean-field continuum is too viscous). In the high-density regime, the bulk limit is approached from below, while as the walls become further apart, the single-particle rescaling is approached from above. Presumably, for wide enough channels, these two limits become indistinguishable.

This line of analysis is suggestive of a 'phase' diagram describing the particle dynamics in terms of either the wall-dominated or bulk-dominated regimes. Of particular interest to researchers (theoreticians and experimentalists alike) should be 


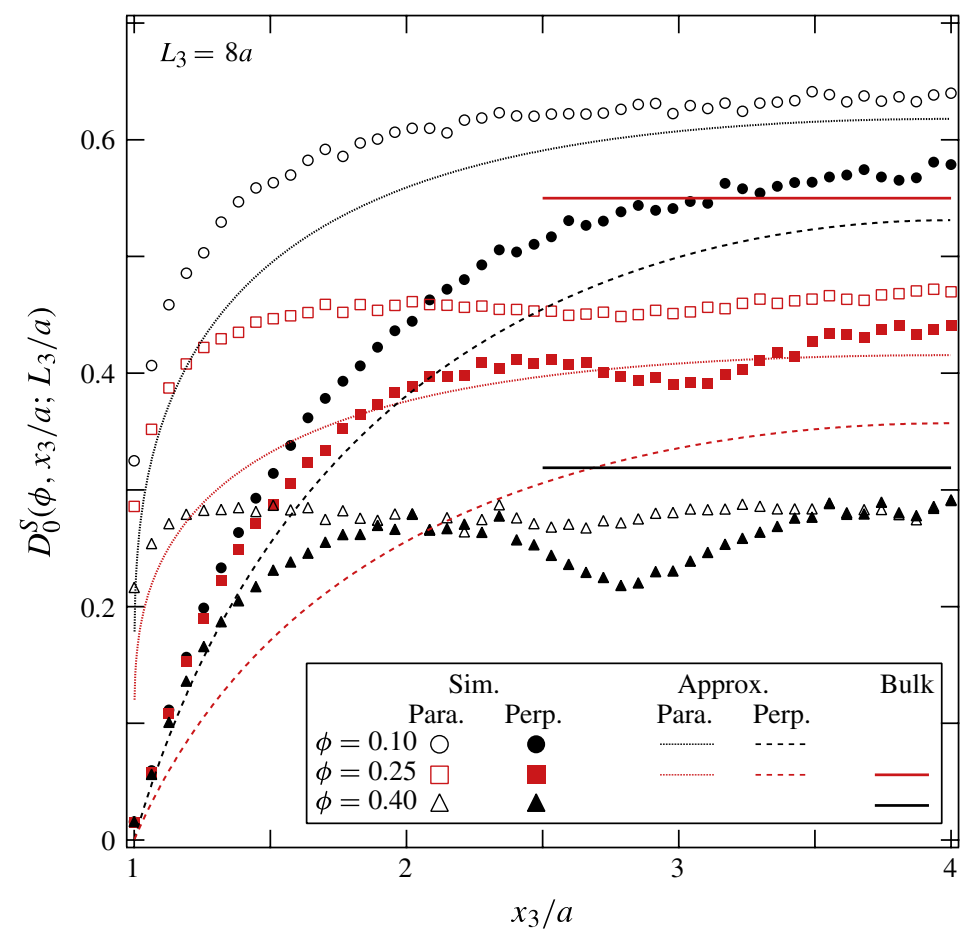

FIGURE 14. (Colour online) The short-time self-diffusivity is measured via simulation and compared to an approximation for the diffusivity of a single particle in the channel for an effective viscosity $k T / 6 \pi a D_{0}^{S}(\phi)$ as well as the bulk short-time self-diffusivity. This is a computation for $L_{3}=8 a$.

the breakdown region where the suspension dynamics cannot be described by any simple combination of 'local' properties. Such a diagram based on the short-time self-diffusivity is sketched in figure 16, though the details remain to be firmly worked through. Indeed, the boundaries of such a diagram are fuzzy and could depend on the property being investigated, though, as a qualitative tool, it may suggest a 'sweetspot' for future studies of confined soft matter. This 'spot' where the dynamics are complicated by an intricate combination of suspension structure and particle-particle and particle-wall hydrodynamic interactions remains largely unstudied. Additionally, there is a region of high confinement that has been widely studied and in which a pseudo-two-dimensional dynamic behaviour is observed (see e.g. Marcus, Lin \& Rice 1996). This is not pursued here, though the hydrodynamic method presented does not preclude such an investigation.

\subsection{Sedimentation rate}

The mean fall speed of particles within a suspension bound in a channel as a function of volume fraction, channel width and position across the channel is determined. In this case, not only is the sedimentation rate anisotropic and inhomogeneous, it also depends on whether the channel is left open, such that there is no back-flow pressure gradient, or closed, such that there is no mean flux of material (particles and fluid) down the channel. The former only occurs for sedimentation parallel to the channel walls. At lower volume fractions, it is the hydrodynamic interactions that dominate the behaviour of the suspension, as the number density of particles across the channel 


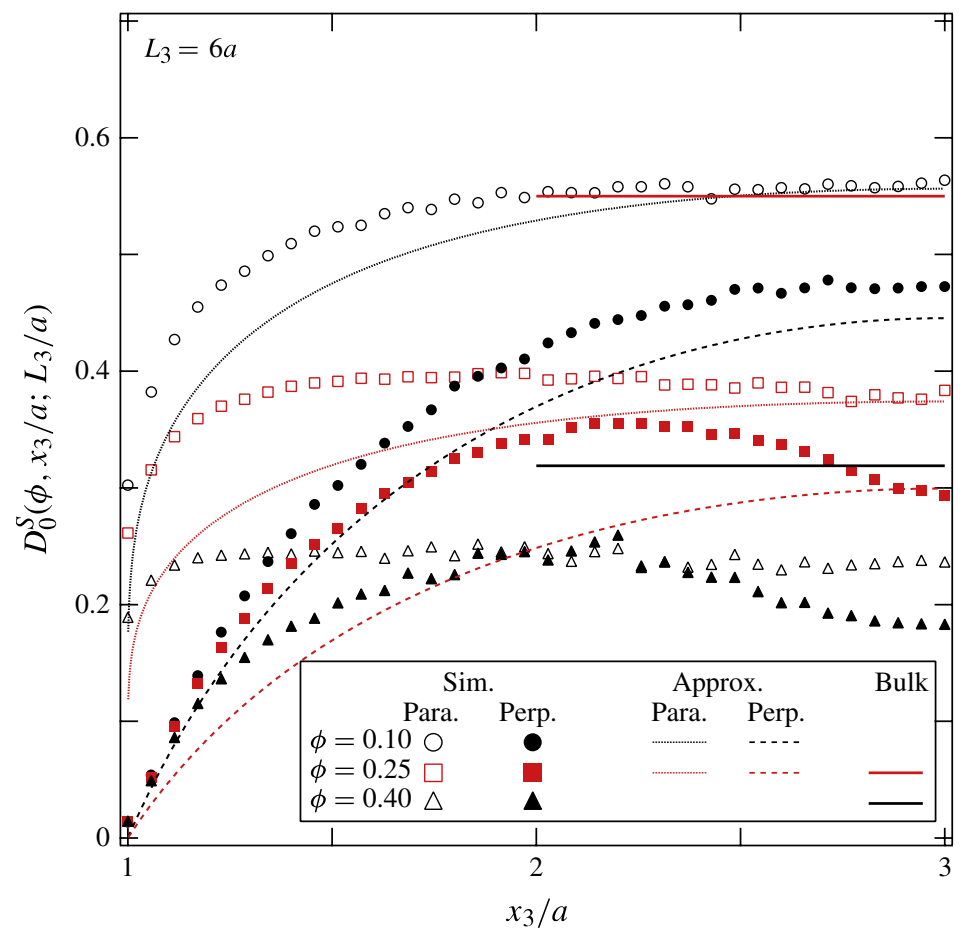

FIGURE 15. (Colour online) The short-time self-diffusivity is measured via simulation and compared to an approximation for the diffusivity of a single particle in the channel for an effective viscosity $k T / 6 \pi a D_{0}^{S}(\phi)$ as well as the bulk short-time self-diffusivity. This is a computation for $L_{3}=6 a$.

is relatively constant. At higher volume fractions, however, the number density of particles varies significantly and therefore so does the local particle flux. This gives rise to different behaviours for different end conditions in the channel. For that matter, a particular volume flux down the channel may be specified and the resulting backflow pressure gradient computed. This constitutes a range of intermediate cases that we do not pursue. The results here correspond to the short-time sedimentation rate of the suspension subject to the equilibrium hard-sphere distribution depicted in figure 9 .

The sedimentation rate $U$ for particles falling down the channel with no net flux and those falling normal to the channel walls due to the force, denoted $F$, are plotted in figure 17 as a function of channel width, volume fraction and position across the channel. At low volume fractions, the particle flux down the channel is effectively parabolic, as might be anticipated. At higher volume fractions, the sedimentation rate varies significantly about a plug flow profile as the mean gravitational body force on the suspension varies directly with the local number density (cf. figure 9). It is the interplay of this local number density variation and the back-flow that gives rise to the variations. The product of the number density and sedimentation rate does not fluctuate, however.

At no point is the sedimentation rate negative. Apparently, the back-flow can never be strong enough to cause particles to levitate rather than fall. Additionally, there is a weak dependence of the sedimentation rate on channel width at low volume fractions. That is, as a function of position across the channel, the sedimentation rate in channels of different widths is nearly indistinguishable at both $10 \%$ and 


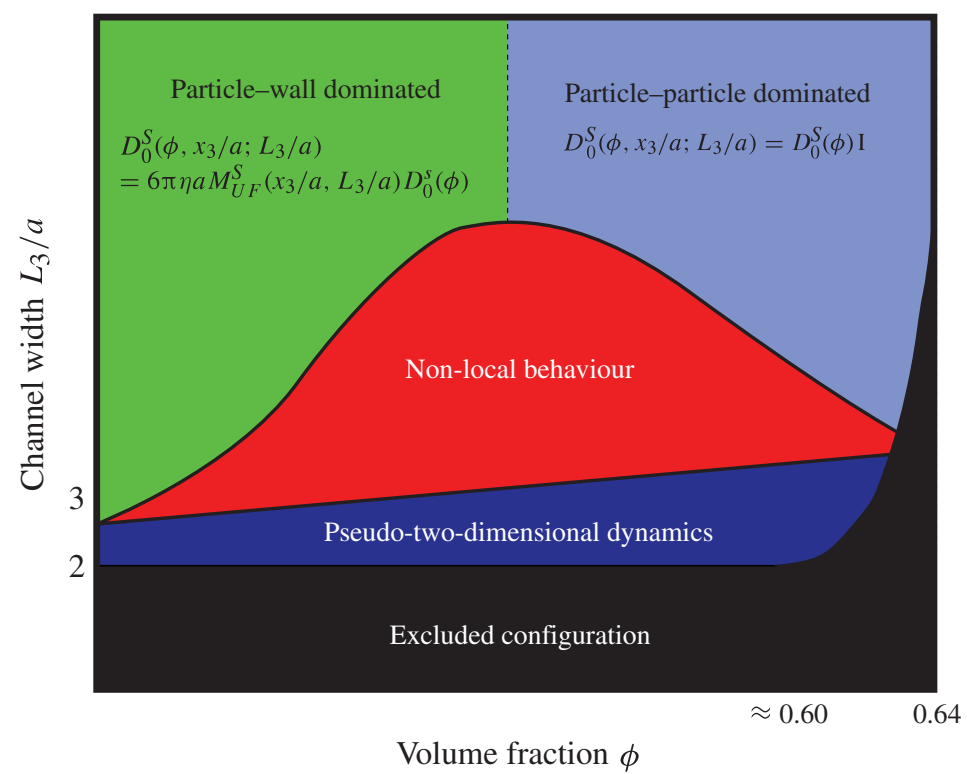

FIGURE 16. (Colour online) A 'phase' diagram based on the short-time self-diffusivity suggesting the dynamical behaviour observed by particles bound by channels of varying widths at varying volume fractions. The key regions are: the particle-wall dominated regime at low density and moderate to large channel widths, for which the dynamics are essentially those of a single particle immersed in a fluid of effective viscosity $k T / 6 \pi a D_{0}^{S}(\phi)$; the particle-particle dominated regime at high concentrations and moderate channel widths, where the dynamics are indistinguishable from the bulk material; and the non-local regime, in which the interplay of particle-particle and particle-wall hydrodynamics are inseparable and the details of suspension structure and hydrodynamics are necessary for accurate prediction of the particle dynamics.

$20 \%$ particle volume fractions. This is what one would expect of Poiseuille flow, where the pressure gradient is independent of the channel width (i.e. the body force on the suspension depends only on the mean density of the suspension). In the normal direction, however, there is a much stronger dependence on the channel width at all volume fractions. This is due to the comparatively stronger hindrance to motion of even a single particle in the normal direction. While one might expect that the particle-particle hydrodynamic interactions are insignificant because of the exponential rate of hydrodynamic screening for motion in the normal direction, the strong variations in the number density and consequently the sedimentation rate at higher volume fractions demonstrate that local, collective motion whose rate is set precisely by those hydrodynamic interactions is not only perceptible but significant. As expected, with increasing particle volume fraction, the sedimentation rate decreases in situations where the mean flux of material is zero.

In figure 18, the sedimentation rate of a suspension along a channel with no restriction on the flux is presented. As would be expected, with no pressure gradient applied down the channel, heavier suspensions fall faster. This statement must be taken in a particular context, though, as the mean particle flux of the heavier suspension is always larger even if the local sedimentation rate appears lower. Additionally, the profile of the sedimentation rate across the channel shows an interesting dependence on the volume fraction. For less concentrated suspensions, the typical parabolic flow 


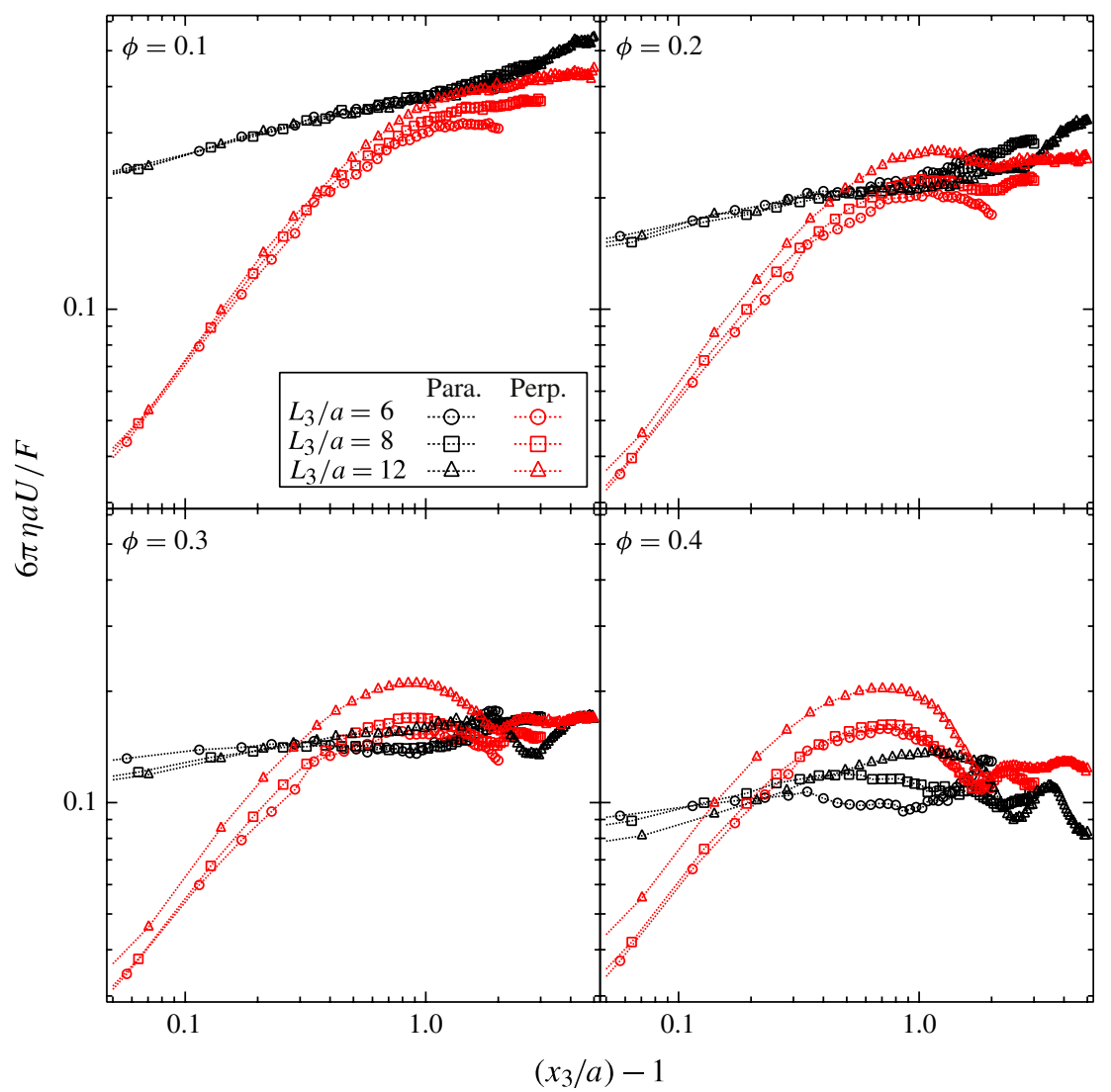

FIgURE 17. (Colour online) Plotted here is the sedimentation rate of a suspension of particles in a sealed channel (i.e. no mean flow) in the directions along and normal to the channel walls. The figure axes are logarithmic so that the dimensionless sedimentation rate is plotted between 0.03 and 0.6 .

profile emerges, while for more concentrated suspensions plug flow prevails. This is intriguing, as observations of this behaviour have been made (see e.g. Nott \& Brady 1994; Lyon \& Leal 1998) for suspensions driven by a pressure gradient down a channel and near steady state with markedly different number density distributions. It appears that, regardless of the distribution, at higher volume fractions, the hydrodynamic screening nullifies the effect of the walls and produces a nearly uniform velocity profile. The uniform velocity profile appears to set in at lower volume fractions for more narrow channels as well. This is probably due to the fact that, for narrower channels, it is the lubrication interactions with the walls that dominate. These are more or less indistinguishable from particle-particle lubrication interactions.

\section{Conclusions}

It is a challenge to account accurately for the low-Reynolds-number hydrodynamic interactions among many particles in a confined geometry. Not only are there the intrinsic length scales set by the bounding geometry, but the many-body interactions are important over different length scales as well. Identifying these length scales, 


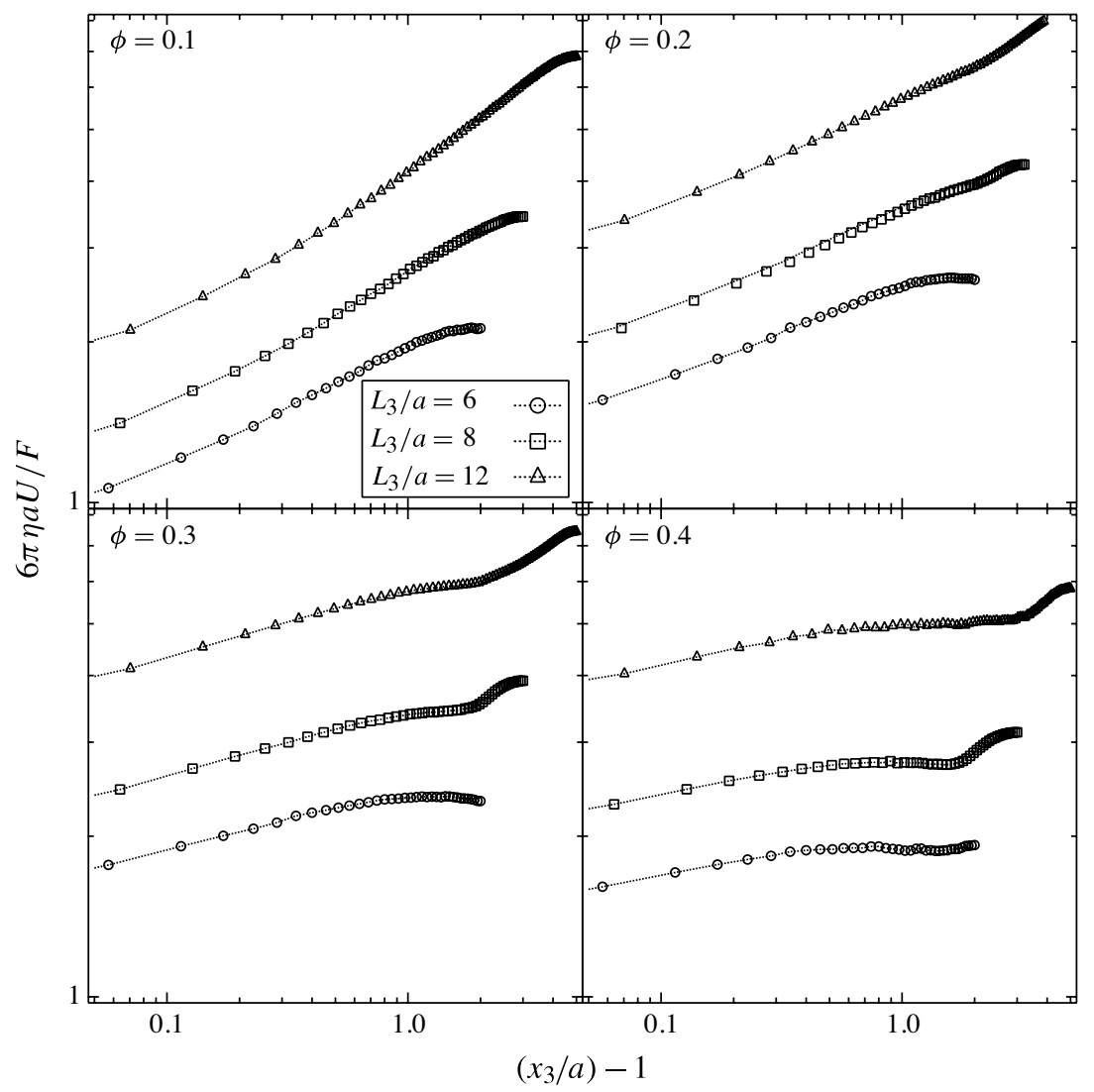

FIGURE 18. The sedimentation rate down a channel with no applied pressure gradient changes from parabolic to uniform profiles with increasing volume fraction while the mass flow rate similarly increases. The figure axes are logarithmic so that the dimensionless sedimentation rate is plotted between 1 and 8 .

minimizing their interdependence and then approximating them with both speed and fidelity is the key not only to reproducing known computations and experiments but also to predicting novel behaviour. In this work, the hydrodynamic interactions among a suspension of particles bound between parallel walls were separated into near-field and far-field components following the typical Stokesian dynamics methodology. Then, the far-field interactions were further divided into those mediated by both channel walls and those mediated by a single wall. This introduces the so-called splitting parameter typical of Ewald summation procedures. By and large, it is the channel width that sets the magnitude of this parameter when the channel is of the order of a few to tens of particle radii wide. The purpose of this scaling is to optimize the summation over interactions among particle pairs. As a consequence of the splitting, the far-field interactions mediated by both channel walls are reproduced exactly while those mediated by a single wall are treated as a superposition approximation, which introduces an exponentially small error. The process of summing over both sets of interactions requires only log-linear time to compute with respect to the number of particles.

The static rheology and short-time dynamics that emerge from the simulations conducted suggest a complex interplay of particle-particle and particle-wall 
hydrodynamic interactions. For the volume fractions and channel widths considered, the density striations throughout the channel play a secondary role. The high-frequency dynamic viscosity deviates little from the value expected for an unbounded suspension. While there is the anticipated dependence on the channel width (the viscosity grows as the channel narrows), the difference between that increment and the bulk value was less than $15 \%$ for all volume fractions and channel widths considered. However, the increment beyond the Einstein contribution to the viscosity $(5 \phi / 2)$ reveals that, at low volume fractions, the particle-wall interactions make the predominant contribution to the viscous dissipation. At high volume fractions, the particle-particle hydrodynamic interactions dominate instead, and the bulk rheology is recovered. This same trend is observed for the short-time self-diffusivity. We find that there is a range of moderate suspension density and channel width for which there is an intimate and inseparable connection between hydrodynamic interactions among the particles and the walls.

While it may be the case that a continuum approach is suitable for modelling suspensions, there is a Knudsen number characterizing the ratio of the relevant macroscopic length scale (i.e. the channel width or distance between the particle and the nearest channel wall) to the particle size, which can be of order one and limits the effectiveness of such an approach. Namely, when the length scale of interest is of the same order of magnitude as the particle-particle spacing, the detailed micromechanics become a necessary part of the model formulation. Similarly though, there are approximations to be made within this regime that can avoid such complexities. When the average distance between the particles, $n\left(x_{3}\right)^{-1 / 3}$, is small relative to a macroscopic length scale, $L_{3}$ for instance, then even though $a / L_{3}$ is of order one, the bulk properties dominate. This corresponds to concentrated suspensions regardless of the channel width. When the opposite is true such that $a / L_{3}$ is of order one while $n\left(x_{3}\right)^{-1 / 3} / L_{3}$ is large, the short-time particle dynamics are the same as those of a single, confined particle in a fluid with effective viscosity $k T / 6 \pi a D_{0}^{S}(\phi)$. Thus the local approximation can be extended with little effort in the case of a Knudsen number of order unity. This, of course, requires further analysis to determine the particular ranges of applicability.

Notice that the short-time dynamics (diffusivity and sedimentation rate) parallel to the channel are sizable in the region near the wall. This begs the question: from a macroscopic perspective what is the appropriate boundary condition on the velocity of the suspension? The empirical no-slip condition employed with great success for Newtonian fluids may not apply at all. Instead, the velocity of the material in a layer near the boundary surely depends on how that material is being deformed. Like the shear viscosity of a suspension serving as the coefficient of proportionality between the bulk shear stress and the bulk rate of strain, it seems reasonable that a slip length characterizes the relationship between the velocity of a layer of suspension near the boundary and the shear stress in that layer. This is the Navier slip condition; however, like the shear viscosity of a suspension, the slip length surely depends on the relative strength of the imposed deformation (i.e. the Péclet number characterizing the ratio of the Brownian time scale to the time scale of the imposed flow). Further investigation is needed.

Future studies will focus on the non-equilibrium rheology and dynamics of confined suspensions. The rule of thumb for short-time dynamics laid out in figure 16 will certainly change with the introduction of a dynamic variable such as the shear rate, sedimentation rate or applied pressure gradient. As the suspension structure was of varied importance to the rheology, diffusivity and sedimentation rate, and this is what will change in a dynamic experiment, it is difficult to predict this change a priori or in 
general. Regardless, the method proposed is suitable for such studies. There is fruitful ground here for looking beyond the suspension's particle-pair structure to larger-scale morphologies, how those change in confinement, and how this affects viscoelastic properties, shear thickening and jamming.

This work was supported in part by NSF grant CBET 0506701.

\section{Appendix A. Confinement by a slipping channel}

The velocity field due to a periodic body force in a channel with shear-stress-free walls is subject to (2.8)-(2.12); however, the solution to the governing differential equations is slightly different, viz.

$$
\begin{aligned}
\boldsymbol{u}^{(k)}\left(x_{3}\right)= & \left(\boldsymbol{I}-2 \pi k x_{3} \boldsymbol{m} \boldsymbol{m}\right) \cdot \boldsymbol{A}^{(k)} \mathrm{e}^{2 \pi k x_{3}} \\
& +\left(\boldsymbol{I}+2 \pi k x_{3} \hat{\boldsymbol{m}} \hat{\boldsymbol{m}}\right) \cdot \boldsymbol{B}^{(k)} \mathrm{e}^{-2 \pi k x_{3}}+\boldsymbol{U}^{(k)}\left(x_{3}\right),
\end{aligned}
$$

for which $\boldsymbol{U}^{(k)}\left(x_{3}\right)$ remains unchanged. The unknown coefficients, $\boldsymbol{A}^{(k)}$ and $\boldsymbol{B}^{(k)}$, are determined by satisfying the no-penetration conditions, $u_{3}^{(k)}(0)=u_{3}^{(k)}\left(L_{3}\right)=0$, and the zero-shear-stress conditions, $\partial_{x_{3}} u_{\alpha}^{(k)}(0)=\partial_{x_{3}} u_{\alpha}^{(k)}\left(L_{3}\right)=0$, for $\alpha=(1,2)$, at the channel walls. As a vector equation, these boundary conditions may be written as

$$
2 \pi k \boldsymbol{e}_{3} \boldsymbol{e}_{3} \cdot \boldsymbol{u}^{(k)}\left(x_{3}\right)+\left(\boldsymbol{I}-\boldsymbol{e}_{3} \boldsymbol{e}_{3}\right) \cdot \frac{\partial \boldsymbol{u}^{(k)}}{\partial x_{3}}=0,
$$

for $x_{3}=0$ and $x_{3}=L_{3}$. The solution of these equations is simplified by writing the vector normal to the walls in terms of $\boldsymbol{m}$ and $\hat{\boldsymbol{m}}$, i.e. $(\boldsymbol{m}+\hat{\boldsymbol{m}}) \cdot \boldsymbol{u}^{(k)}\left(x_{3}\right)=0$ and $(\boldsymbol{m}-\hat{\boldsymbol{m}}) \cdot \partial_{x_{3}} \boldsymbol{u}^{(k)}\left(x_{3}\right)=0$ when $x_{3}=0, L_{3}$. The resulting coefficients are

$$
\begin{aligned}
\boldsymbol{m} \cdot \boldsymbol{A}^{(k)}= & -\hat{\boldsymbol{m}} \cdot \boldsymbol{B}^{(k)}=-\frac{1}{12 \pi k} \operatorname{csch}\left(2 \pi k L_{3}\right)\left[\boldsymbol{m} \cdot\left(2 \pi k \boldsymbol{U}^{(k)}\left(L_{3}\right)+\left.\frac{\partial \boldsymbol{U}^{(k)}}{\partial x_{3}}\right|_{L_{3}}\right)\right. \\
& \left.+\hat{\boldsymbol{m}} \cdot\left(2 \pi k \boldsymbol{U}^{(k)}\left(L_{3}\right)-\left.\frac{\partial \boldsymbol{U}^{(k)}}{\partial x_{3}}\right|_{L_{3}}\right)\right] \\
\hat{\boldsymbol{m}} \cdot \boldsymbol{A}^{(k)}= & -\boldsymbol{m} \cdot \boldsymbol{B}^{(k)}=-\frac{1}{3 \pi k} \mathrm{e}^{4 \pi k L_{3}}\left(1-\mathrm{e}^{4 \pi k L_{3}}\right)^{-2} \\
& \times\left\{2 \pi k L _ { 3 } \operatorname { c o s h } ( 2 \pi k L _ { 3 } ) \left[\boldsymbol{m} \cdot\left(2 \pi k \boldsymbol{U}^{(k)}\left(L_{3}\right)+\left.\frac{\partial \boldsymbol{U}^{(k)}}{\partial x_{3}}\right|_{L_{3}}\right)\right.\right. \\
& \left.+\hat{\boldsymbol{m}} \cdot\left(2 \pi k \boldsymbol{U}^{(k)}\left(L_{3}\right)-\left.\frac{\partial \boldsymbol{U}^{(k)}}{\partial x_{3}}\right|_{L_{3}}\right)\right] \\
& +\sinh \left(2 \pi k L_{3}\right)\left[\boldsymbol{m} \cdot\left(4 \pi k \boldsymbol{U}^{(k)}\left(L_{3}\right)-\left.\frac{\partial \boldsymbol{U}^{(k)}}{\partial x_{3}}\right|_{L_{3}}\right)\right. \\
& \left.\left.+\hat{\boldsymbol{m}} \cdot\left(4 \pi k \boldsymbol{U}^{(k)}\left(L_{3}\right)+\left.\frac{\partial \boldsymbol{U}^{(k)}}{\partial x_{3}}\right|_{L_{3}}\right)\right]\right\}
\end{aligned}
$$

while

$$
\tilde{\boldsymbol{m}} \cdot \boldsymbol{A}^{(k)}=\tilde{\boldsymbol{m}} \cdot \boldsymbol{B}^{(k)}=-\left.\frac{1}{4 \pi k} \operatorname{csch}\left(2 \pi k L_{3}\right) \tilde{\boldsymbol{m}} \cdot \frac{\partial \boldsymbol{U}^{(k)}}{\partial x_{3}}\right|_{L_{3}},
$$


where $\tilde{\boldsymbol{m}}=(2 \pi k)^{-1}\left(2 \pi k_{2} \boldsymbol{e}_{1}-2 \pi k_{1} \boldsymbol{e}_{2}\right)$ and $\boldsymbol{I}=\boldsymbol{m} \boldsymbol{m}+\hat{\boldsymbol{m}} \hat{\boldsymbol{m}}+\tilde{\boldsymbol{m}} \tilde{\boldsymbol{m}}$. Since the particular solution to the Stokes equations is independent of the boundary conditions, the procedures introduced in $\$ 2.3$ are equally applicable. One necessary change is in the reflection of the local contribution to the velocity field. The analogue to (2.39) for the velocity field generated by a Stokeslet above a zero-shear-stress boundary is simply

$$
\boldsymbol{u}_{l}(\boldsymbol{x})=\int_{V}[\boldsymbol{G}(\boldsymbol{r} ; \alpha)-\boldsymbol{G}(\boldsymbol{R} ; \alpha)] \cdot \boldsymbol{f}\left(\boldsymbol{x}^{\prime}\right) \mathrm{d} \boldsymbol{x}^{\prime} .
$$

With this, similar computations of the motion of particles in a shear-stress-free channel are straightforward. Before concluding this section, consider a channel bounded above and below by another fluid with viscosity denoted $\eta_{w}$. The velocity field in this channel, $\boldsymbol{u}(\boldsymbol{x})$, can always be written as a linear superposition of the flow in a no-slip channel, $\boldsymbol{u}_{n s}(\boldsymbol{x})$, and the flow in a zero-shear-stress channel, $\boldsymbol{u}_{s}(\boldsymbol{x})$, such that

$$
\boldsymbol{u}(\boldsymbol{x})=\frac{\lambda}{1+\lambda} \boldsymbol{u}_{s}(\boldsymbol{x})+\frac{1}{1+\lambda} \boldsymbol{u}_{n s}(\boldsymbol{x}),
$$

where $\lambda=\eta / \eta_{w}$ (see e.g. Lee, Chadwick \& Leal 1979). This linear superposition makes the simulation of particle motion in channels bounded by viscous fluids accessible as well.

\section{Appendix B. Integrals for computing the particular solution to the global Stokes equations}

The global contribution to the velocity field due to a set of particles is dictated by the particular solution to the Stokes equations subject to the body force in (2.42). This particular solution involves the computation of the series of integrals shown in (2.16)-(2.18), which are represented as

$$
\begin{aligned}
& \boldsymbol{C}_{1}^{(k)}\left(x_{3}\right)=\sum_{n} \mathrm{e}^{2 \pi \mathrm{i} k_{\alpha} x_{\alpha}^{(n)}} D_{1}^{(n)}\left(x_{3}\right) \boldsymbol{F}_{n}, \\
& \boldsymbol{C}_{2}^{(k)}\left(x_{3}\right)=\sum_{n} \mathrm{e}^{2 \pi \mathrm{i} k_{\alpha} x_{\alpha}^{(n)}} D_{2}^{(n)}\left(x_{3}\right) \boldsymbol{F}_{n}, \\
& \boldsymbol{C}_{3}^{(k)}\left(x_{3}\right)=\sum_{n} \mathrm{e}^{2 \pi \mathrm{i} k_{\alpha} x_{\alpha}^{(n)}} D_{3}^{(n)}\left(x_{3}\right) \boldsymbol{F}_{n} .
\end{aligned}
$$

Since the notion of discrete forcing in the fluid has been introduced, the solution to the Stokes equations becomes a linear superposition of these forces and the necessary integrals simplify, viz.

$$
\begin{aligned}
D_{1}^{(n)}\left(x_{3}\right)= & \frac{\sqrt{\alpha}}{8 \pi}\left\{2 \mathrm{e}^{-\pi \alpha k^{2}-\pi \Delta x_{3}^{(n) 2} / \alpha}\right. \\
& -\mathrm{e}^{-\pi \alpha k^{2}-\pi x_{3}^{(n) 2} / \alpha}\left[\left(1+\frac{x_{3}^{(n)}}{\alpha k}\right) \mathrm{e}^{2 \pi k x_{3}}+\left(1-\frac{x_{3}^{(n)}}{\alpha k}\right) \mathrm{e}^{-2 \pi k x_{3}}\right] \\
& -\frac{\mathrm{e}^{2 \pi k \Delta x_{3}^{(n)}}}{\sqrt{\alpha} k}\left[\operatorname{erf}\left(\sqrt{\pi \alpha} k+\sqrt{\frac{\pi}{\alpha}} \Delta x_{3}^{(n)}\right)-\operatorname{erf}\left(\sqrt{\pi \alpha} k-\sqrt{\frac{\pi}{\alpha}} x_{3}^{(n)}\right)\right]
\end{aligned}
$$




$$
\begin{aligned}
& -\frac{\mathrm{e}^{-2 \pi k \Delta x_{3}^{(n)}}}{\sqrt{\alpha} k}\left[\operatorname { e r f } \left(\sqrt{\pi \alpha} k-\sqrt{\left.\frac{\pi}{\alpha} \Delta x_{3}^{(n)}\right)}\right.\right. \\
& \left.\left.-\operatorname{erf}\left(\sqrt{\pi \alpha} k+\sqrt{\frac{\pi}{\alpha}} x_{3}^{(n)}\right)\right]\right\} \\
D_{2}^{(n)}\left(x_{3}\right)= & \frac{\sqrt{\alpha}}{8 \pi}\left\{\mathrm{e}^{-\pi \alpha k^{2}-\pi \Delta x_{3}^{(n) 2} / \alpha}-\mathrm{e}^{-\pi \alpha k^{2}-\pi x_{3}^{(n) 2} / \alpha}\left[1-2 \pi k x_{3}\left(1+\frac{x_{3}^{(n)}}{\alpha k}\right)\right] \mathrm{e}^{2 \pi k x_{3}}\right. \\
& +\frac{2 \pi \Delta x_{3}^{(n)}}{\sqrt{\alpha}} \mathrm{e}^{2 \pi k \Delta x_{3}^{(n)}}\left[\operatorname{erf}\left(\sqrt{\pi \alpha} k+\sqrt{\frac{\pi}{\alpha}} \Delta x_{3}^{(n)}\right)\right. \\
& \left.\left.-\operatorname{erf}\left(\sqrt{\pi \alpha} k-\sqrt{\frac{\pi}{\alpha}} x_{3}^{(n)}\right)\right]\right\}, \\
& \frac{\sqrt{\alpha}}{8 \pi}\left\{\mathrm{e}^{-\pi \alpha k^{2}-\pi \Delta x_{3}^{(n) 2} / \alpha}-\mathrm{e}^{-\pi \alpha k^{2}-\pi x_{3}^{(n) 2} / \alpha}\left[1-2 \pi k x_{3}\left(1+\frac{x_{3}^{(n)}}{\alpha k}\right)\right] \mathrm{e}^{-2 \pi k x_{3}}\right. \\
& -\frac{2 \pi \Delta x_{3}^{(n)}}{\sqrt{\alpha}} \mathrm{e}^{-2 \pi k \Delta x_{3}^{(n)}}\left[\operatorname { e r f } \left(\sqrt{\pi \alpha} k-\sqrt{\left.\frac{\pi}{\alpha} \Delta x_{3}^{(n)}\right)}\right.\right. \\
& \left.\left.-\operatorname{erf}\left(\sqrt{\pi \alpha} k+\sqrt{\frac{\pi}{\alpha}} x_{3}^{(n)}\right)\right]\right\} .
\end{aligned}
$$

The functions, $\boldsymbol{C}_{i}^{(k)}\left(\boldsymbol{x}_{3}\right)$, are weighted Fourier transformations of the forces in the fluid, where the weighting factors are the values of $D_{i}^{(n)}\left(x_{3}\right)$ as indicated in (B 1)-(B 3). This interpretation lends itself to direct computation via the fast Fourier transform technique.

\section{Appendix C. Reflections of the local velocity field}

For an approximation of the local contribution to the velocity field, one may treat the channel walls independently. Equation (2.37) expresses the local velocity field due to the reflection off the lower wall as a convolution of the local force density with the Stokeslet and its reflection. Integrals such as those in (2.38) for $\boldsymbol{G}\left(\boldsymbol{r}^{\prime \prime} ; \alpha\right)$ are necessary. This is the contribution due to the Stokeslet. The contribution due to the reflected Stokeslet is broken into three pieces such that

$$
\begin{gathered}
\int_{V} \boldsymbol{J}_{w}\left(\boldsymbol{R}, x_{3}^{\prime}\right)\left[\delta\left(\boldsymbol{r}^{\prime}\right)-\frac{1}{\alpha^{3 / 2}}\left(\frac{5}{2}-\frac{\pi r^{\prime 2}}{\alpha}\right) \mathrm{e}^{-\left(\pi r^{\prime 2} / \alpha\right)}\right] \mathrm{d} \boldsymbol{x}^{\prime} \\
=-\boldsymbol{G}\left(\boldsymbol{R}^{\prime \prime} ; \alpha\right)+\boldsymbol{G}_{w}^{(1)}\left(\boldsymbol{R}^{\prime \prime}, x_{3}^{\prime \prime} ; \alpha\right)+\boldsymbol{G}_{w}^{(2)}\left(\boldsymbol{R}^{\prime \prime}, x_{3}^{\prime \prime} ; \alpha\right),
\end{gathered}
$$

where

$$
\begin{aligned}
\boldsymbol{G}_{w}^{(1)}\left(\boldsymbol{R}^{\prime \prime}, x_{3}^{\prime \prime} ; \alpha\right)= & \nabla_{x}^{2} \int_{V} x_{3}^{\prime 2} \boldsymbol{J}(\boldsymbol{R})\left[\delta\left(\boldsymbol{r}^{\prime}\right)-\frac{1}{\alpha^{3 / 2}}\left(\frac{5}{2}-\frac{\pi r^{\prime 2}}{\alpha}\right) \mathrm{e}^{-\left(\pi r^{\prime 2} / \alpha\right)}\right] \mathrm{d} \boldsymbol{x}^{\prime} \\
& \times\left(\boldsymbol{I}-2 \boldsymbol{e}_{3} \boldsymbol{e}_{3}\right)
\end{aligned}
$$

and

$$
\boldsymbol{G}_{w}^{(2)}\left(\boldsymbol{R}^{\prime \prime}, x_{3}^{\prime \prime} ; \alpha\right)=-2\left\{\left(\boldsymbol{I}-2 \boldsymbol{e}_{3} \boldsymbol{e}_{3}\right) \cdot \nabla_{x} \int_{V} x_{3}^{\prime} \boldsymbol{J}(\boldsymbol{R})\right.
$$




$$
\left.\times\left[\delta\left(\boldsymbol{r}^{\prime}\right)-\frac{1}{\alpha^{3 / 2}}\left(\frac{5}{2}-\frac{\pi r^{\prime 2}}{\alpha}\right) \mathrm{e}^{-\left(\pi r^{\prime 2} / \alpha\right)}\right] \mathrm{d} \boldsymbol{x}^{\prime} \cdot \boldsymbol{e}_{3}\right\}^{\mathrm{T}} .
$$

Again, in order to avoid computing these integrals directly, one simply writes them in terms of $\boldsymbol{G}\left(\boldsymbol{R}^{\prime \prime} ; \alpha\right)$. This is accomplished by taking the appropriate derivatives of $\boldsymbol{G}\left(\boldsymbol{R}^{\prime \prime} ; \alpha\right)$ with respect to $x_{3}^{\prime \prime}$ such that

$$
\begin{aligned}
\boldsymbol{H}\left(\boldsymbol{R}^{\prime \prime} ; \alpha\right)= & \int_{V} x_{3}^{\prime} \boldsymbol{J}(\boldsymbol{R})\left[\delta\left(\boldsymbol{r}^{\prime}\right)-\frac{1}{\alpha^{3 / 2}}\left(\frac{5}{2}-\frac{\pi r^{\prime 2}}{\alpha}\right) \mathrm{e}^{-\left(\pi r^{\prime 2} / \alpha\right)}\right] \mathrm{d} \boldsymbol{x}^{\prime} \\
= & x_{3}^{\prime \prime} \boldsymbol{G}\left(\boldsymbol{R}^{\prime \prime} ; \alpha\right)+\frac{\alpha}{2 \pi} \frac{\partial}{\partial x_{3}^{\prime \prime}}\left[\boldsymbol{G}\left(\boldsymbol{R}^{\prime \prime} ; \alpha\right)+\boldsymbol{J}\left(\boldsymbol{R}^{\prime \prime}\right)\right] \\
& +\frac{1}{\alpha^{3 / 2}} \int_{V}\left(x_{3}^{\prime}-x_{3}^{\prime \prime}\right) \boldsymbol{J}(\boldsymbol{R}) \mathrm{e}^{-\left(\pi r^{\prime 2} / \alpha\right)} \mathrm{d} \boldsymbol{x}^{\prime},
\end{aligned}
$$

and similarly

$$
\begin{gathered}
\int_{V} x_{3}^{\prime 2} \boldsymbol{J}(\boldsymbol{R})\left[\delta\left(\boldsymbol{r}^{\prime}\right)-\frac{1}{\alpha^{3 / 2}}\left(\frac{5}{2}-\frac{\pi r^{\prime 2}}{\alpha}\right) \mathrm{e}^{-\left(\pi r^{\prime 2} / \alpha\right)}\right] \mathrm{d} \boldsymbol{x}^{\prime} \\
=x_{3}^{\prime \prime} \boldsymbol{H}\left(\boldsymbol{R}^{\prime \prime} ; \alpha\right)+\frac{\alpha}{2 \pi} \frac{\partial}{\partial x_{3}^{\prime \prime}}\left[\boldsymbol{H}\left(\boldsymbol{R}^{\prime \prime} ; \alpha\right)-x_{3}^{\prime \prime} \boldsymbol{J}\left(\boldsymbol{R}^{\prime \prime} ; \alpha\right)\right] \\
+\frac{1}{\alpha^{3 / 2}} \int_{V} x_{3}^{\prime}\left(x_{3}^{\prime}-x_{3}^{\prime \prime}\right) \boldsymbol{J}(\boldsymbol{R}) \mathrm{e}^{-\left(\pi r^{\prime 2} / \alpha\right)} \mathrm{d} \boldsymbol{x}^{\prime} .
\end{gathered}
$$

What remains then is to compute the integrals in (C4) and (C5). Again, these are difficult to compute in general. One must begin with a convolution of the Stokeslet with a Gaussian,

$$
\boldsymbol{v}(\boldsymbol{x})=\frac{1}{\alpha^{3 / 2}}\left(\int_{V} \boldsymbol{J}(\boldsymbol{R}) \mathrm{e}^{-\left(\pi r^{\prime 2} / \alpha\right)} \mathrm{d} \boldsymbol{x}^{\prime}\right) \cdot \boldsymbol{F},
$$

from which a physical interpretation becomes rather obvious. This is the velocity field, $\boldsymbol{v}(\boldsymbol{x})$, at $\boldsymbol{x}$ due to a Gaussian-distributed body force of magnitude $\boldsymbol{F}$ centred on $\boldsymbol{x}^{\prime \prime}-2 x_{3}^{\prime \prime} \boldsymbol{e}_{3}$ and is the solution to the equations:

$$
\begin{gathered}
\eta \nabla_{x}^{2} \boldsymbol{v}(\boldsymbol{x})=\nabla p-\frac{1}{\alpha^{3 / 2}} \mathrm{e}^{-\left(\pi R^{\prime \prime 2} / \alpha\right)} \boldsymbol{F}, \\
\boldsymbol{\nabla} \cdot \boldsymbol{v}(\boldsymbol{x})=0 .
\end{gathered}
$$

This is solved using the standard Fourier transform technique (see e.g. Kim \& Karrila 2005) and a relatively simple expression for the integral in (C6) is found:

$$
\begin{aligned}
\boldsymbol{v}(\boldsymbol{x})= & \frac{1}{4 \pi \eta}\left(\frac{1}{4 \pi \alpha^{1 / 2} R^{\prime \prime 3}}\right)\left\{\boldsymbol{I}\left[2 \alpha^{1 / 2}\left(2 \pi R^{\prime \prime 2}-\alpha\right)-4 \pi R^{\prime \prime 3}\left(\tilde{\phi}_{-1 / 2}-\tilde{\phi}_{1 / 2}\right)\right]\right. \\
& \left.-\left(\boldsymbol{I}-\hat{\boldsymbol{R}}^{\prime \prime} \hat{\boldsymbol{R}}^{\prime \prime}\right)\left[\alpha^{1 / 2}\left(2 \pi R^{\prime 2}-3 \alpha\right)+2 \pi R^{\prime \prime 3}\left(\tilde{\phi}_{-1 / 2}-3 \tilde{\phi}_{1 / 2}\right)\right]\right\} \cdot \boldsymbol{F},
\end{aligned}
$$

where

$$
\tilde{\phi}_{k}=\phi_{k}\left(\frac{\pi R^{\prime 2}}{\alpha}\right)
$$


For reference, the identities below were derived for this purpose:

$$
\int_{V} \mathrm{e}^{2 \pi i x \cdot \xi} f(\xi) \mathrm{d} \xi=\frac{2}{r} \int_{0}^{\infty} \xi \sin (2 \pi \xi r) f(\xi) \mathrm{d} \xi
$$

and

$$
\int_{V} \mathrm{e}^{2 \pi i x \cdot \xi} f(\xi) \hat{\xi} \hat{\xi} \mathrm{d} \xi=X_{1} \boldsymbol{I}+X_{2} \hat{\boldsymbol{x}} \hat{\boldsymbol{x}}
$$

where

$$
\begin{gathered}
X_{1}=\int_{0}^{\infty}\left(2 \pi^{2} r^{3} \xi\right)^{-1}[\sin (2 \pi \xi r)-2 \pi \xi r \cos (2 \pi \xi r)] f(\xi) \mathrm{d} \xi \\
X_{2}=\int_{0}^{\infty}\left(2 \pi^{2} r^{3} \xi\right)^{-1}\left[6 \pi \xi r \cos (2 \pi \xi r)-\left(3-(2 \pi \xi r)^{2}\right) \sin (2 \pi \xi r)\right] f(\xi) \mathrm{d} \xi
\end{gathered}
$$

both of which are absolutely convergent for all $f(\xi) \sim o\left(\xi^{-2}\right)$ as $\xi \rightarrow 0$. With this, it is straightforward to show that

$$
\begin{aligned}
\frac{1}{\alpha^{3 / 2}} \int_{V} x_{3}^{\prime} \boldsymbol{J}(\boldsymbol{R}) \mathrm{e}^{-\pi r^{\prime 2} / \alpha}= & \frac{1}{4 \pi \eta}\left(\frac{1}{8 \pi^{2} \alpha^{1 / 2} R^{\prime \prime 7}}\right)\left\{\boldsymbol { I } \left[x _ { 3 } ^ { \prime \prime } \left(2 \pi \alpha^{1 / 2} R^{\prime \prime 4}\left[2 \pi R^{\prime \prime 2}+\alpha\right]\right.\right.\right. \\
& \left.-4 \pi^{2} R^{\prime \prime 7}\left[\tilde{\phi}_{-1 / 2}+\tilde{\phi}_{1 / 2}\right]\right)-R_{3}^{\prime \prime}\left(\alpha^{3 / 2} R^{\prime 2}\left[2 \pi R^{\prime \prime 2}+3 \alpha\right]\right. \\
& \left.\left.-4 \pi^{2} R^{\prime \prime 7}\left[\tilde{\phi}_{1 / 2}+\tilde{\phi}_{3 / 2}\right]\right)\right]+\left(\boldsymbol{R}^{\prime \prime} \boldsymbol{e}_{3}+\boldsymbol{e}_{3} \boldsymbol{R}^{\prime \prime}\right)\left[\alpha^{3 / 2} R^{\prime 2}\left(2 \pi R^{\prime \prime 2}-3 \alpha\right)\right. \\
& \left.-2 \pi \alpha R^{\prime \prime 5}\left[\tilde{\phi}_{-1 / 2}-3 \tilde{\phi}_{1 / 2}\right]\right]+\boldsymbol{R}^{\prime \prime} \boldsymbol{R}^{\prime \prime}\left[x _ { 3 } ^ { \prime \prime } \left(2 \pi \alpha^{1 / 2} R^{\prime 2}\left[2 \pi R^{\prime 2}-6 \alpha\right]\right.\right. \\
& \left.-4 \pi R^{\prime \prime 5}\left[\tilde{\phi}_{-1 / 2}-3 \tilde{\phi}_{1 / 2}\right]\right)-R_{3}^{\prime \prime}\left(3 \alpha^{3 / 2}\left[2 \pi R^{\prime \prime 2}-5 \alpha\right]\right. \\
& \left.\left.\left.-4 \pi R^{\prime \prime 3}\left[\alpha \tilde{\phi}_{-1 / 2}+\left(\pi R^{\prime \prime 2}-3 \alpha\right) \tilde{\phi}_{1 / 2}-3 \pi R^{\prime \prime 2} \tilde{\phi}_{3 / 2}\right]\right)\right]\right\}, \quad(\mathrm{C} 15)
\end{aligned}
$$

and

$$
\begin{aligned}
& \frac{1}{\alpha^{3 / 2}} \int_{V} x_{3}^{\prime}\left(x_{3}^{\prime}-x_{3}^{\prime \prime}\right) \boldsymbol{J}(\boldsymbol{R}) \mathrm{e}^{-\left(\pi r^{\prime 2} / \alpha\right)}=\frac{1}{4 \pi \eta}\left(\frac{1}{16 \pi^{3} \alpha^{1 / 2} R^{\prime \prime 9}}\right)\left\{\boldsymbol { I } \left[4 \alpha^{3 / 2} R^{\prime \prime 4}\left(\pi^{2} R^{\prime \prime 4}-3 \alpha^{2}\right)\right.\right. \\
& -4 \pi^{2} R^{\prime \prime 9}\left(\tilde{\phi}_{-1 / 2}-\tilde{\phi}_{3 / 2}\right)-x_{3}^{\prime \prime} R_{3}^{\prime \prime}\left(2 \pi \alpha^{3 / 2} R^{\prime \prime 4}\left[2 \pi R^{\prime \prime 2}+3 \alpha\right]\right. \\
& \left.-8 \pi^{3} R^{\prime \prime 9}\left[\tilde{\phi}_{1 / 2}+\tilde{\phi}_{3 / 2}\right]\right)+R_{3}^{\prime \prime 2}\left(3 \alpha^{5 / 2} R^{\prime \prime 2}\left[2 \pi R^{\prime \prime 2}+5 \alpha\right]\right. \\
& \left.\left.-8 \pi^{3} R^{\prime \prime 9}\left[\tilde{\phi}_{3 / 2}+\tilde{\phi}_{5 / 2}\right]\right)\right]+\boldsymbol{e}_{3} \boldsymbol{e}_{3}\left[2 \alpha^{5 / 2} R^{\prime \prime 4}\left(2 \pi R^{\prime \prime 2}-3 \alpha\right)\right. \\
& \left.-4 \pi \alpha^{2} R^{\prime \prime 7}\left(\tilde{\phi}_{-1 / 2}-3 \tilde{\phi}_{1 / 2}\right)\right]+\left(\boldsymbol{R}^{\prime \prime} \boldsymbol{e}_{3}+\boldsymbol{e}_{3} \boldsymbol{R}^{\prime \prime}\right)\left[x _ { 3 } ^ { \prime \prime } \left(2 \pi \alpha^{3 / 2} R^{\prime \prime 4}\left[2 \pi R^{\prime \prime 2}-3 \alpha\right]\right.\right. \\
& \left.-2 \pi^{2} \alpha R^{\prime \prime 7}\left[\tilde{\phi}_{-1 / 2}-3 \tilde{\phi}_{1 / 2}\right]\right)-R_{3}^{\prime \prime}\left(6 \alpha^{5 / 2} R^{\prime \prime 2}\left[2 \pi R^{\prime \prime 2}-5\right]\right. \\
& \left.\left.-8 \pi \alpha R^{\prime \prime 5}\left[\alpha \tilde{\phi}_{-1 / 2}+\left(\pi R^{\prime \prime 2}-3 \alpha\right) \tilde{\phi}_{1 / 2}-3 \pi R^{\prime \prime 2} \tilde{\phi}_{3 / 2}\right]\right)\right] \\
& -\boldsymbol{R}^{\prime \prime} \boldsymbol{R}^{\prime \prime}\left[3 \alpha^{5 / 2} R^{\prime \prime 2}\left(2 \pi R^{\prime 2}-5 \alpha\right)\right. \\
& -2 \pi \alpha^{3 / 2} R^{\prime \prime 4}\left(2 \pi R^{\prime 2}-3 \alpha\right)+4 \pi \alpha R^{\prime \prime 5}\left(\left[\pi R^{\prime 2}-\alpha\right] \tilde{\phi}_{-1 / 2}\right. \\
& \left.-\left[4 \pi R^{\prime \prime 2}+3 \alpha\right] \tilde{\phi}_{1 / 2}+3 \pi R^{\prime \prime 2} \tilde{\phi}_{3 / 2}\right)+x_{3}^{\prime \prime} R_{3}^{\prime \prime}\left(6 \pi \alpha^{3 / 2} R^{\prime \prime 2}\left[2 \pi R^{\prime \prime 2}-5 \alpha\right]\right. \\
& \left.-8 \pi^{2} R^{\prime \prime 5}\left[\alpha \tilde{\phi}_{-1 / 2}+\left(\pi R^{\prime \prime 2}-3 \alpha\right) \tilde{\phi}_{1 / 2}-3 \pi R^{\prime \prime 2} \tilde{\phi}_{3 / 2}\right]\right) \\
& -R_{3}^{\prime \prime 2}\left(15 \alpha^{5 / 2}\left[2 \pi R^{\prime \prime 2}-7 \alpha\right]-8 \pi \alpha R^{\prime \prime 3}\left[2 \alpha \tilde{\phi}_{-1 / 2}\right.\right. \\
& \left.\left.\left.\left.+2 \pi R^{\prime 2}\left(\pi R^{\prime 2}-3 \alpha\right) \tilde{\phi}_{1 / 2}+\left(\pi R^{\prime \prime 2}-6 \alpha\right) \tilde{\phi}_{3 / 2}-3 \pi^{2} R^{\prime \prime 4} \tilde{\phi}_{5 / 2}\right]\right)\right]\right\},
\end{aligned}
$$


by respectively taking the derivative once and twice with respect to $x_{3}^{\prime \prime}$. After some tedious algebra, during which the results from (C4), (C 5) and (C9) are combined, the reflection tensor can be computed. This is available as supplementary material at journals.cambridge.org/flm; however, a few salient details are described here. The reflection tensor scales as $\exp \left(-\pi R^{2} / \alpha\right)$, as might be expected. However, neither the doublet nor source-doublet contributions to the reflection have that same exponential scaling. This is of critical importance, since it is only the sum of these two terms weighted exactly as described by Blake (1971) that has the appropriate scaling. As is usually the case with Stokes flow, there is almost no middle ground between the simplest approximations and fully developed solutions. An approximation that neglects or mis-weights either of these terms will not decay exponentially and the entire argument for splitting the disturbance velocity into two contributions becomes invalid.

This essentially concludes the derivation of the single-wall reflection of the realspace contribution to the Ewald-like force density in the fluid. It is worth noting, however, that, if the radius of curvature of the wall is large relative to $\sqrt{\alpha}$, it may be possible to employ this result in simulations of non-planar bounding geometries. The reason for this is obvious. Since the force density decays exponentially fast for small values of $\sqrt{\alpha}$, a wall with a sufficiently large radius of curvature appears to be locally flat. This should be rather useful for studying the motion of particles in a tube (see e.g. Liron 1984) as well as particles in other curvilinear confinements.

\section{Appendix D. Wave-space contributions to the 'self'-mobility tensors}

Because the global disturbance velocity determined in these simulations is combined with Faxén formulae to compute the grand mobility tensor, care must be taken that the 'self'-contribution is not over-counted. That is, each particle must not sense its own contribution to the global disturbance flow. Therefore, this contribution is computed explicitly a priori and the appropriate Faxén formulae applied so that, for each particle $n$ a given fractional distance across the channel, denoted $\Xi$, and for a given dimensionless splitting parameter $\beta=\alpha / L_{3}^{2}$, the wave-space contribution to the self-mobility tensors is removed from any calculations. To simplify things, the torque and stresslet are summed into a generalized doublet denoted $\boldsymbol{D}$, and the rotation and rate of strain are summed into a generalized velocity gradient denoted $\boldsymbol{\nabla} \boldsymbol{u}(\boldsymbol{x})$. Through symmetry arguments and fluid incompressibility, it can be shown that the coupling between velocity and doublet takes the form

$$
\begin{gathered}
\boldsymbol{M}_{U D}^{S}=\frac{1}{6 \pi \eta a_{n}^{2}}\left\{\left[f_{2}^{(U D)}(\Xi, \beta)\left(\frac{a_{n}}{L_{3}}\right)^{2}-f_{4}^{(U D)}(\Xi, \beta)\left(\frac{a_{n}}{L_{3}}\right)^{4}+f_{6}^{(U D)}(\Xi, \beta)\left(\frac{a_{n}}{L_{3}}\right)^{6}\right] \boldsymbol{I} \boldsymbol{e}_{3}\right. \\
+\left[g_{2}^{(U D)}(\Xi, \beta)\left(\frac{a_{n}}{L_{3}}\right)^{2}-g_{4}^{(U D)}(\Xi, \beta)\left(\frac{a_{n}}{L_{3}}\right)^{4}+g_{6}^{(U D)}(\Xi, \beta)\left(\frac{a_{n}}{L_{3}}\right)^{6}\right]\left(\boldsymbol{e}_{3} \boldsymbol{I}-2 \boldsymbol{e}_{3} \boldsymbol{e}_{3} \boldsymbol{e}_{3}\right) \\
\left.+\left[h_{2}^{(U D)}(\Xi, \beta)\left(\frac{a_{n}}{L_{3}}\right)^{2}-h_{4}^{(U D)}(\Xi, \beta)\left(\frac{a_{n}}{L_{3}}\right)^{4}+h_{6}^{(U D)}(\Xi, \beta)\left(\frac{a_{n}}{L_{3}}\right)^{6}\right] \boldsymbol{I} \boldsymbol{e}_{3}^{\mathrm{T}}\right\}, \quad(\mathrm{D} 1)
\end{gathered}
$$

while the coupling between velocity gradient and doublet can be written as

$$
\begin{aligned}
\boldsymbol{M}_{\nabla D}= & \frac{1}{6 \pi \eta a_{n}^{3}}\left\{-\left[f_{3}^{(E S)}(\Xi, \beta)\left(\frac{a_{n}}{L_{3}}\right)^{3}-f_{5}^{(E S)}(\Xi, \beta)\left(\frac{a_{n}}{L_{3}}\right)^{5}+f_{7}^{(E S)}(\Xi, \beta)\left(\frac{a_{n}}{L_{3}}\right)^{7}\right]\right. \\
& \times\left[\left(\delta_{i j}-\delta_{i 3} \delta_{j 3}\right)\left(\delta_{k l}-\delta_{k 3} \delta_{l 3}\right)-2\left(\delta_{i j}-\delta_{i 3} \delta_{j 3}\right) \delta_{k 3} \delta_{l 3}\right.
\end{aligned}
$$




$$
\begin{aligned}
& \left.-2 \delta_{i 3} \delta_{j 3}\left(\delta_{k l}-\delta_{k 3} \delta_{l 3}\right)+4 \delta_{i 3} \delta_{j 3} \delta_{k 3} \delta_{l 3}\right] \\
& -\left[g_{3}^{(E S)}(\Xi, \beta)\left(\frac{a_{n}}{L_{3}}\right)^{3}-g_{5}^{(E S)}(\Xi, \beta)\left(\frac{a_{n}}{L_{3}}\right)^{5}+g_{7}^{(E S)}(\Xi, \beta)\left(\frac{a_{n}}{L_{3}}\right)^{7}\right] \\
& \times\left[\left(\delta_{i k}-\delta_{i 3} \delta_{k 3}\right)\left(\delta_{j l}-\delta_{j 3} \delta_{l 3}\right)+\left(\delta_{i l}-\delta_{i 3} \delta_{l 3}\right)\left(\delta_{j k}-\delta_{j 3} \delta_{k 3}\right)\right. \\
& \left.-2\left(\delta_{i j}-\delta_{i 3} \delta_{j 3}\right) \delta_{k 3} \delta_{l 3}-2 \delta_{i 3} \delta_{j 3}\left(\delta_{k l}-\delta_{k 3} \delta_{l 3}\right)+4 \delta_{i 3} \delta_{j 3} \delta_{k 3} \delta_{l 3}\right] \\
& -\left[h_{3}^{(E S)}(\Xi, \beta)\left(\frac{a_{n}}{L_{3}}\right)^{3}-h_{5}^{(E S)}(\Xi, \beta)\left(\frac{a_{n}}{L_{3}}\right)^{5}+h_{7}^{(E S)}(\Xi, \beta)\left(\frac{a_{n}}{L_{3}}\right)^{7}\right] \\
& \times\left[\left(\delta_{i k}-\delta_{i 3} \delta_{k 3}\right) \delta_{j 3} \delta_{l 3}+\left(\delta_{i l}-\delta_{i 3} \delta_{l 3}\right) \delta_{j 3} \delta_{k 3}+\left(\delta_{j k}-\delta_{j 3} \delta_{k 3}\right) \delta_{i 3} \delta_{l 3}\right. \\
& \left.\left.+\left(\delta_{j l}-\delta_{j 3} \delta_{l 3}\right) \delta_{i 3} \delta_{k 3}\right]\right\} \boldsymbol{e}_{i} \boldsymbol{e}_{j} \boldsymbol{e}_{k} \boldsymbol{e}_{l} .
\end{aligned}
$$

coupling between velocity gradient and force is simply the transpose of the velocitydoublet coupling. These are easily computed in much the same fashion as the selfcontribution to $\boldsymbol{M}_{U F}$ from the wave-space disturbance flow. Plotting them is not instructive, however, and further discussion of the details and resulting tabulations may be undertaken through correspondence with the authors.

Supplementary material available at journals.cambridge.org/flm.

\section{REFERENCES}

Ali, I., Marenduzzo, D. \& Yeomans, J. M. 2004 Dynamics of polymer packaging. J. Chem. Phys. 121 (17), 8635-8642.

Allen, M. P. \& Tildesley, D. J. 1989 Computer Simulation of Liquids. Oxford University Press.

Ashton, R., Padala, C. \& Kane, R. S. 2003 Microfluidic separation of DNA. Current Opinions in Biotechnology 14 (5), 497-504.

Beenakker, C. W. J. 1986 Ewald sum of the Rotne-Prager tensor. J. Chem. Phys. 85 (3), $1581-1582$.

BhatTACHARYA, S. 2008 Cooperative motion of spheres arranged in periodic grids between two walls. J. Chem. Phys. 128, 074709.

Bhattacharya, S. \& BlawZdZIEWICZ, J. 2002 Image system for Stokes-flow singularity between two parallel planar walls. J. Math. Phys. 43 (11), 5720-5731.

Bhattacharya, S., Blawzdziewicz, J. \& Wajnryb, E. 2005 Hydrodynamic interactions of spherical particles in suspensions confined between two planar walls. J. Fluid Mech. 541, 263-292.

Blake, J. R. 1971 A note on the image system for a Stokeslet in a no-slip boundary. Proc. Camb. Phil. Soc. 70, 303-310.

Bossis, G., Meunier, A. \& Sherwood, J. D. 1991 Stokesian dynamics simulations of particle trajectoriers near a plane. Phys. Fluids A 3 (8), 1853-1858.

Brady, J. F. \& Bossis, G. 1988 Stokesian dynamics. Annu. Rev. Fluid Mech. 20, 111-157.

Brady, J. F, Phillips, R. J., Lester, J. C. \& Bossis, G. 1988 Dynamic simulation of hydrodynamically interacting suspensions. J. Fluid Mech. 195, 257-280.

Butler, J. E. \& BonneCAZE, R. T. 1999 Imaging of particle shear migration with electrical impedance tomography. Phys. Fluids 11 (8), 1982.

Cichocki, B., Jones, R. B., Kutteh, R. \& Wajnryb, E. 2000 Friction and mobility for colloidal spheres in Stokes flow near a boundary: the multipole method and applications. $J$. Chem. Phys. 112 (5), 2548-2561.

Cox, R. G. \& HsU, S. K. 1977 The lateral migration of solid particles in a laminar flow near a plane. Intl J. Multiphase Flow 3 (3), 201-222. 
Cox, R. G. \& Mason, S. G. 1971 Suspended particles in fluid flow through tubes. Annu. Rev. Fluid Mech. 3, 291-316.

Daniels, B. R., Masi, B. C. \& WiRTZ, D 2006 Probing single-cell micromechanics in vivo: the microrheology of C. Elegans developing embryos. Biophys. J. 90 (12), 4712-4719.

Darden, T., York, D. \& Pedersen, L. 1993 Particle mesh Ewald: an $N \cdot \log (N)$ method for Ewald sums in large systems. J. Chem. Phys. 98 (12), 10089-10093.

Donev, A., Stillinger, F. H. \& Torquato, S. 2005 Neighbor list collision-driven molecular dynamics simulation for non-spherical particles. I. Algorithmic details II. Applications to ellipses and ellipsoids. J. Comput. Phys. 202 (2), 737-793.

Dufresne, E. R., Altman, D. \& Grier, D. G. 2001 Brownian dynamics of a sphere between parallel walls. Europhys. Lett. 53 (2), 264-270.

Durlofsky, L. J. \& BRADY, J. F. 1989 Dynamic simulation of bounded suspensions of hydrodynamically interacting particles. J. Fluid Mech. 200, 39-67.

FAXÉN, H. 1921 Dissertations, Uppsala University. Ark. Mat. Astron. Fys. 17, 27.

Frank, M., Anderson, D., Weeks, E. R. \& Morris, J. F. 2003 Particle migration in pressure-driven flow of a Brownian suspension. J. Fluid Mech. 493, 363-378.

Frenkel, D. \& Smit, B. 2001 Understanding Molecular Simulations, 2nd edn. Academic Press.

DE Gennes, P. G. 1979 Conjectures on the transition from Poiseuille to plug flow in suspensions. J. Phys. 40 (8), 783-787.

Glendinning, A. B. \& Russel, W. B. 1982 A pairwise additive description of sedimentation and diffusion in concentrated suspensions of hard spheres. J. Colloid Interface Sci. 89 (1), $123-143$.

Goldsmith, H. L. \& Mason, S. G. 1962 The of suspensions through tubes. I. Single spheres, rods and discs. J. Colloid Sci. 17 (5), 448-476.

Gregory, J. 1981 Flocculation in laminar tube flow. Chem. Engng Sci. 36 (11), 1789-1794.

Guth, E. \& Simha, R. 1936 Untersuchungen über die viskosität von suspensionen und lösungen. 3. Über die viskosität von kugelsuspensionen. Colloid Polym. Sci. 74 (3), 266.

Happel, J. \& Brenner, H. 1986 Low Reynolds Number Hydrodynamics, 2nd edn. Prentice-Hall.

Hалімото, H. 1959 On the periodic fundamental solutions of the Stokes equations and their application to viscous flow past a cubic array of spheres. J. Fluid Mech. 5 (2), 317-328.

Hernández-Ortiz, J. P., de Pablo, J. J. \& Graham, M. D. 2007 Fast computation of many-particle hydrodynamic and electrostatic interactions in a confined geometry. Phys. Rev. Lett. 98 (14), 140602.

Hockney, R. W. \& Eastwood, J. W. 1989 Computer Simulation Using Particles. Taylor and Francis.

Jones, R. B. 2004 Spherical particle in Poiseuille flow between planar walls. J. Chem. Phys. 121, 483-500.

Karnis, A., Goldsmith, H. L. \& MAson, S. G. 1966 The kinetics of flowing dispersions: I. Concentrated suspensions of rigid particles. J. Colloid Interface Sci. 22 (6), 521-553.

KIM, S. \& KARRILA, S. J. 2005 Microhydrodynamics, 2nd edn. Dover Publications.

Koн, C. J., Hoокham, P. \& LeAL, L. G. 1994 An experimental investigation of concentrated suspension flows in a rectangular channel. J. Fluid Mech. 266, 1-32.

LAdYZHENSKAYA, O. A. 1963 The Mathematical Theory of Viscous Incompressible Flow. Gordon and Breach.

Lee, S. H., Chadwick, R. S. \& Leal, L. G. 1979 Motion of a sphere in the presence of a plane interface. Part 1: an approximate solution by generalization of the method of Lorentz. J. Fluid Mech. 93 (4), 705-726.

Leighton, D. T. \& ACrivos, A. 1987 Measurement of shear-induced self-diffusion in concentrated suspensions of spheres. J. Fluid Mech. 177, 109-131.

LiRON, N. 1984 Stokeslet arrays in a pipe and their application to ciliary transport. J. Fluid Mech. 143, 173-195.

Liron, N. \& Mochon, S. 1976 Stokes flow for a Stokeslet between two parallel flat plates. J. Engng Maths 10, 287-303.

LyON, M. K. \& LEAL, L. G. 1998 An experimental study of the motion of concentrated suspensions in two-dimensional channel flow. J. Fluid Mech. 363, 25-56. 
Marcus, A. H., LiN, B. \& RICE, S. A. 1996 Self-diffusion in quasi-two-dimension hard sphere suspensions. Phys. Rev. E 53 (2), 1765-1776.

Norman, J. T., Nayak, H. V. \& Bonnecaze, R. T. 2005 Migration of buoyant particles in low-Reynolds-number pressure driven flows. J. Fluid Mech. 523, 1-35.

NotT, P. R. \& BRADY, J. F. 1994 Pressure driven flow of suspensions: simulation and theory. J. Fluid Mech. 275, 157-199.

O'Neill, M. E. \& Stewartson, K. 1967 On the slow motion of a sphere parallel to a nearby plane wall. J. Fluid Mech. 27 (4), 705-724.

Oseen, C. W. 1928 Neuere Methoden und Ergebnisse in der Hydrodynamik. Dissertation, Akademische Verlagsgesellschaft, Leipzig.

Phung, T. N., Brady, J. F. \& Bossis, G. 1996 Stokesian dynamics simulation of Brownian suspensions. J. Fluid Mech. 313, 181-207.

RAMACHANDRAN, A. \& LEIGHTON, D. T. 2007 Viscous resuspension in a tube: the impact of secondary flows resulting from second normal stress differences. Phys. Fluids 19 (5), 053301.

Sierou, A. \& Brady, J. F. 2001 Accelerated Stokesian dynamics simulations. J. Fluid Mech. 448, $115-146$.

SeShadRI, V. \& SUtera, S. P. 1970 Apparent viscosity of coarse concentrated suspensions in tube flow. J. Rheol. 14 (3), 351-373.

Staben, M. E., Zinchenko, A. Z. \& Davis, R. H. 2003 Motion of a particle between two parallel plane walls in low-Reynolds-number Poiseuille flow. Phys. Fluids 15, 1711-1734.

SWAN, J. W. \& BRADY, J. F. 2007 Simulation of hydrodynamically interacting particles near a no-slip boundary. Phys. Fluids 19, 113306.

SWAN, J. W. \& BRADY, J. F. 2010 Particle motion between parallel walls: hydrodynamics and simulation. Phys. Fluids 22, 103301.

Zarraga, I. E. \& Leighton, D. T. 2002 Measurement of an unexpectedly large shear-induced self-diffusivity in a dilute suspension of spheres. Phys. Fluids 14 (7), 2194.

Zurita-Gotor, M., Blawzdziewicz, J. \& Wajnryb, E. 2007 Motion of a rod-like particle between parallel walls with application to suspension rheology. J. Rheol. 51 (1), 71-97. 